Published in:

"Celestial Mechanics and Dynamical Astronomy" (2005) 91:75-108

\title{
Long-term evolution of orbits about a precessing* oblate planet: \\ 1. The case of uniform precession.
}

\author{
Michael Efroimsky \\ US Naval Observatory, Washington DC 20392 USA \\ e-mail: me @ usno.navy.mil
}

August 2, 2018

\begin{abstract}
It was believed until very recently that a near-equatorial satellite would always keep up with the planet's equator (with oscillations in inclination, but without a secular drift). As explained in Efroimsky and Goldreich (2004), this misconception originated from a wrong interpretation of a (mathematically correct) result obtained in terms of non-osculating orbital elements. A similar analysis carried out in the language of osculating elements will endow the planetary equations with some extra terms caused by the planet's obliquity change. Some of these terms will be nontrivial, in that they will not be amendments to the disturbing function. Due to the extra terms, the variations of a planet's obliquity may cause a secular drift of its satellite orbit inclination. In this article we set out the analytical formalism for our study of this drift. We demonstrate that, in the case of uniform precession, the drift will be extremely slow, because the first-order terms responsible for the drift will be short-period and, thus, will have vanishing orbital averages (as anticipated 40 years ago by Peter Goldreich), while the secular terms will be of the second order only. However, it turns out that variations of the planetary precession make the first-order terms secular. For example, the planetary nutations will resonate with the satellite's orbital frequency and, thereby, may instigate a secular drift. A detailed study of this process will be offered in a subsequent publication, while here we work out the required mathematical formalism and point out the key aspects of the dynamics.
\end{abstract}

* In this article, we use the word "precession" in its most general sense which embraces the entire spectrum of changes of the spin-axis orientation - from the long-term variations down to the Chandler-type wobble down to nutations and to the polar wonder. 


\section{Physical motivation and the statement of purpose}

Ward $(1973,1974)$ noted that the obliquity of Mars may have suffered large-angle motions at long time scales. Later, Laskar and Robutel (1993) and Touma and Wisdom (1994) demonstrated that these motions may have been chaotic. This would cause severe climate variations and have major consequences for development of life.

It is a customary assumption that a near-equatorial satellite of an oblate planet would always keep up with the planet's equator (with only small oscillations of the orbit inclination) provided the obliquity changes are sufficiently slow (Goldreich 1965, Kinoshita 1993). As demonstrated in Efroimsky and Goldreich (2004), this belief stems from a calculation performed in the language of non-osculating orbital elements. A similar analysis carried out in terms of osculating elements will contain hitherto overlooked extra terms entailed by the planet's obliquity variations. These terms (emerging already in the first order over the precession-caused perturbation) will cause a secular angular drift of the satellite orbit away from the planetary equator.

The existence of Phobos and Deimos, and the ability of Mars to keep them close to its equatorial plane during obliquity variations sets constraints on the obliquity variation amplitude and rate. Our eventual goal is to establish such constraints. If the satellites' secular inclination drifts are slow enough that the satellites stay close to Mars' equator during its obliquity changes through billions of years, then the rigid-planet non-dissipative models used by Ward (1973, 1974), Laskar \& Robutel (1993), and Touma \& Wisdom (1994) will get a totally independent confirmation. If the obliquity-variation-caused inclination drifts are too fast (fast enough that within a billion or several billions of years the satellites get driven away from Mars' equatorial plane), then the inelastic dissipation and planetary structure must play a larger role than previously assumed.

Having this big motivation in mind, we restrict the current article to building the required mathematical background: we study the obliquity-variation-caused terms in the planetary equations, calculate their secular components and point out the resonant coupling emerging between a satellite's orbiting frequency and certain frequencies in the planet axis' precession. A more thorough investigation of this interaction will be left for our next paper.

\section{Mathematical preliminaries: osculating elements vs orbital elements}

Whenever one embarks on integrating a satellite orbit and wants to take into account direction variations of the planet's spin, it is most natural to carry out this work in a co-precessing coordinate system. This always yields orbital elements defined in the said frame, which are ready for immediate physical interpretation by a planet-based observer. A well camouflaged pitfall of this approach is that these orbital elements may come out non-osculating, i.e., that the instantaneous ellipses (or hyperbolae) parametrised by these elements will not be tangent to the physical trajectory as seen in the said frame of reference. 


\subsection{The osculation condition and alternatives to it}

An instantaneous orbit is parametrised with six independent Keplerian parameters. These include the three Eulerian angles $i, \omega, \Omega$ which define the orientation of the instantaneous orbital plane, the eccentricity and semimajor axis of the instantaneous ellipse or hyperbola, and the mean anomaly at an epoch, $M_{o}$, which determines the initial position of the body. Often are employed sets of other variables. The variables are always six in number and are some functions of the Keplerian elements. These are, for example, the Delaunay set, the Jacobi set, and two sets offered by Poincare. More exotic is the Hill set which includes the true anomaly as one of the elements (Hill 1913).

Systems of planetary equations in terms of the above sets of parameters may be derived not only through the variation-of-parameters (VOP) method but also by means of the Hamilton-Jacobi one. However, the latter approach, though fine and elegant, lacks the power instilled into the direct VOP technique and cannot account for the gauge invariance of the N-body problem (Efroimsky 2002a,b), important feature intimately connected with some general concepts in ODE (Newman \& Efroimsky 2003). The Hamilton-Jacobi technique implicitly fixes the gauge, and thus leaves the internal symmetry heavily veiled (Efroimsky \& Goldreich 2003). Below we shall present several relevant facts and formulae, while a more comprehensive treatment of the matter may be looked up in (Efroimsky \& Goldreich 2003, 2004).

As well known, a solution

$$
\overrightarrow{\mathbf{r}}=\overrightarrow{\boldsymbol{f}}\left(C_{1}, \ldots, C_{6}, t\right)
$$

to the reduced two-body problem

$$
\ddot{\overrightarrow{\mathbf{r}}}+\frac{G m}{r^{2}} \frac{\overrightarrow{\mathbf{r}}}{r}=0
$$

is a Keplerian ellipse or hyperbola parameterised with some set of six independent orbital elements $C_{i}$ which are constants in the absence of disturbances.

In the N-particle setting (or, more generally, in the presence of whatever disturbances) each body will be subject to some perturbing force $\Delta \overrightarrow{\mathbf{F}}(\overrightarrow{\boldsymbol{r}}, \dot{\vec{r}}, t)$ acting at it from bodies other than the primary:

$$
\ddot{\overrightarrow{\mathbf{r}}}+\frac{G m}{r^{2}} \frac{\overrightarrow{\mathbf{r}}}{r}=\Delta \overrightarrow{\mathbf{F}},
$$

Solving the above equation of motion by the VOP method implies the use of (1) as an ansatz,

$$
\overrightarrow{\mathbf{r}}=\overrightarrow{\boldsymbol{f}}\left(C_{1}(t), \ldots, C_{6}(t), t\right),
$$

the function $\vec{f}$ being the same as in (11), and the "constants" $C_{i}$ now being endowed with a time dependence of their own. Insertion of (4) into (3) is insufficient for determining the six functions $C_{i}(t)$. because the vector equation (3) comprises only three scalar equalities. To furnish a solution three more equations are needed. Since the age of Lagrange it has been advised in the literature to employ for this purpose the conditions of osculation,

$$
\sum_{i} \frac{\partial \overrightarrow{\boldsymbol{f}}}{\partial C_{i}} \frac{d C_{i}}{d t}=0
$$

imposition whereof ensures that the physical velocity,

$$
\frac{d \overrightarrow{\boldsymbol{r}}}{d t}=\frac{\partial \overrightarrow{\boldsymbol{f}}}{\partial t}+\sum_{i} \frac{\partial \overrightarrow{\boldsymbol{f}}}{\partial C_{i}} \frac{d C_{i}}{d t}
$$


in the disturbed case is expressed by the same function $\overrightarrow{\mathrm{g}}\left(C_{1}, \ldots, C_{6}, t\right)$ as it used to in the two-body configuration:

$$
\overrightarrow{\mathrm{g}}=\frac{\partial \overrightarrow{\boldsymbol{f}}}{\partial t}
$$

The conditions being essentially arbitrary, their choice affects only the mathematical developments but not the physical orbit. The orbit's invariance under the alternative choices of the supplementary constraints reflects the gauge freedom, i.e., the internal symmetry present in this problem. In the language of pure mathematics this is a fiber-bundle structure. In physicists' terms it is an example of the gauge freedom. Essentially, this is merely a case of ambiguous parameterisation.

The Lagrange constraint and the law of motion, (3), with ansatz (44) inserted therein, yields the following equation of the elements' evolution:

$$
\sum_{j}\left[C_{n} C_{j}\right] \frac{d C_{j}}{d t}=\frac{\partial \overrightarrow{\boldsymbol{f}}}{\partial C_{n}} \Delta \overrightarrow{\mathbf{F}}
$$

$\left[C_{n} C_{j}\right]$ being the matrix of Lagrange brackets introduced as

$$
\left[C_{n} C_{j}\right] \equiv \frac{\partial \overrightarrow{\boldsymbol{f}}}{\partial C_{n}} \frac{\partial \overrightarrow{\mathbf{g}}}{\partial C_{j}}-\frac{\partial \overrightarrow{\boldsymbol{f}}}{\partial C_{j}} \frac{\partial \overrightarrow{\mathbf{g}}}{\partial C_{n}}
$$

So defined, the brackets depend neither on the time evolution of $C_{i}$ nor on the choice of supplementary conditions, but solely on the functional form of $\overrightarrow{\boldsymbol{f}}\left(C_{1, \ldots, 6}, t\right)$ and $\overrightarrow{\boldsymbol{g}} \equiv \partial \overrightarrow{\boldsymbol{f}} / \partial t$.

In case we decide to relax the Lagrange constraint and to substitute for it

$$
\sum_{i} \frac{\partial \overrightarrow{\boldsymbol{f}}}{\partial C_{i}} \frac{d C_{i}}{d t}=\overrightarrow{\boldsymbol{\Phi}}\left(C_{1, \ldots, 6}, t\right)
$$

$\vec{\Phi}$ being some arbitrary function of time and parameters $C_{i}$ (but, for the reasons of sheer convenience, not of time derivatives of $C_{i}$ ), then instead of (8) we shall get, for $n=1, \ldots, 6$,

$$
\sum_{j}\left(\left[C_{n} C_{j}\right]+\frac{\partial \overrightarrow{\boldsymbol{f}}}{\partial C_{n}} \frac{\partial \overrightarrow{\boldsymbol{\Phi}}}{\partial C_{j}}\right) \frac{d C_{j}}{d t}=\frac{\partial \overrightarrow{\boldsymbol{f}}}{\partial C_{n}} \Delta \overrightarrow{\mathbf{F}}-\frac{\partial \overrightarrow{\boldsymbol{f}}}{\partial C_{n}} \frac{\partial \overrightarrow{\boldsymbol{\Phi}}}{\partial t}-\frac{\partial \overrightarrow{\mathbf{g}}}{\partial C_{n}} \overrightarrow{\boldsymbol{\Phi}}
$$

These gauge-invariant perturbation equations of celestial mechanics, generated by direct application of the VOP method, were derived in (Efroimsky 2002b). They give us an opportunity to use, in an arbitrary gauge $\overrightarrow{\boldsymbol{\Phi}}$, the Lagrange brackets (9) defined in terms of the unperturbed functions $\overrightarrow{\boldsymbol{f}}$ and $\overrightarrow{\mathrm{g}}$. Expressions for these brackets have long been known, and they can be employed to write down the planetary equations in their gauge-invariant form. If our goal were to arrive to the customary Lagrange system of planetary equations, we would now stick to the trivial gauge (5) and restrict ourselves to conservative forces solely: $\Delta \overrightarrow{\mathbf{F}}=\partial R(\overrightarrow{\boldsymbol{r}}) / \partial \overrightarrow{\boldsymbol{r}}$. We however bear in mind the objective of exploring the interplay of two freedoms: freedom of reference frame choice and that of gauge fixing. For this reason not only shall we leave the gauge arbitrary but shall also reserve for our disturbance a form general enough to include both physical and inertial forces, the latter showing themselves en route from an inertial coordinate system to an accelerated one. An example at hand is a satellite orbiting a precessing oblate planet: the oblateness will give birth to an extra physical force, while the precession will result in inertial forces if we decide to describe the 
orbit in non-inertial axes associated with the precessing planet. What is important about the inertial inputs is that they are not only position but also velocity dependent. This gives us a motivation to consider velocity-dependent disturbances. Another motivation for this comes from the relativity where the correction to Newton' gravity law contains velocities (Brumberg 1992)

Expression (11) is the most general form of the equations for the orbital elements, in terms of the disturbing force. To get the generic form of the equations in terms of the Lagrangian disturbance, let us recall that the (reduced) two-body problem is described by the unit-mass Lagrangian $\mathcal{L}_{o}(\overrightarrow{\mathbf{r}}, \dot{\overrightarrow{\mathbf{r}}}, t)=\dot{\overrightarrow{\mathbf{r}}}^{2} / 2-U(\overrightarrow{\mathbf{r}}, t)$, canonical momentum $\overrightarrow{\mathbf{p}}=\dot{\overrightarrow{\mathbf{r}}}$, and Hamiltonian $\mathcal{H}_{o}(\overrightarrow{\mathbf{r}}, \overrightarrow{\mathbf{p}}, t)=\overrightarrow{\mathbf{p}}^{2} / 2+U(\overrightarrow{\mathbf{r}}, t)$, while the disturbed setting will be described by the perturbed functions:

$$
\begin{aligned}
\mathcal{L}(\overrightarrow{\mathbf{r}}, \dot{\overrightarrow{\mathbf{r}}}, t)=\mathcal{L}_{o}+\Delta \mathcal{L}=\frac{\dot{\overrightarrow{\mathbf{r}}}^{2}}{2}-U(\overrightarrow{\mathbf{r}}, t)+\Delta \mathcal{L}(\overrightarrow{\mathbf{r}}, \dot{\overrightarrow{\mathbf{r}}}, t) \\
\overrightarrow{\mathbf{p}}=\dot{\overrightarrow{\mathbf{r}}}+\frac{\partial \Delta \mathcal{L}}{\partial \dot{\overrightarrow{\mathbf{r}}}}
\end{aligned}
$$

and

$$
\begin{gathered}
\mathcal{H}(\overrightarrow{\mathbf{r}}, \overrightarrow{\mathbf{p}}, t)=\overrightarrow{\mathbf{p}} \dot{\overrightarrow{\mathbf{r}}}-\mathcal{L}=\frac{\overrightarrow{\mathbf{p}}^{2}}{2}+U+\Delta \mathcal{H}, \\
\Delta \mathcal{H}(\overrightarrow{\mathbf{r}}, \overrightarrow{\mathbf{p}}, t) \equiv-\Delta \mathcal{L}-\frac{1}{2}\left(\frac{\partial \Delta \mathcal{L}}{\partial \dot{\overrightarrow{\mathbf{r}}}}\right)^{2},
\end{gathered}
$$

$\Delta \mathcal{H}$ being a variation of the functional form: $\Delta \mathcal{H} \equiv \mathcal{H}(\overrightarrow{\mathbf{r}}, \overrightarrow{\mathbf{p}}, t)-\mathcal{H}_{o}(\overrightarrow{\mathbf{r}}, \overrightarrow{\mathbf{p}}, t)$. The Euler-Lagrange equations written for the perturbed Lagrangian will read:

$$
\ddot{\overrightarrow{\mathbf{r}}}=-\frac{\partial U}{\partial \overrightarrow{\mathbf{r}}}+\Delta \overrightarrow{\mathbf{F}}
$$

the new term

$$
\Delta \overrightarrow{\mathbf{F}} \equiv \frac{\partial \Delta \mathcal{L}}{\partial \overrightarrow{\mathbf{r}}}-\frac{d}{d t}\left(\frac{\partial \Delta \mathcal{L}}{\partial \dot{\overrightarrow{\mathbf{r}}}}\right)
$$

being the disturbing force. We see that, in the case of velocity-dependent perturbations, this force is equal neither to the gradient of the Lagrangian's perturbation nor to the gradient of negative Hamiltonian's perturbation. This should be taken into account when comparing results obtained by different techniques. For example, in Goldreich (1965) the word "disturbing function" was used for the negative perturbation of the Hamiltonian. The gradient of so defined disturbing function was not equal to the disturbing force.

Substitution of (16) into (11) then yields the generic form of the equations in terms of the Lagrangian disturbance 1 The result,

\footnotetext{
${ }^{1}$ Direct plugging of (16) into (11) results in

$$
\sum_{j}\left[C_{n} C_{j}\right] \frac{d C_{j}}{d t}=\frac{\partial \overrightarrow{\boldsymbol{f}}}{\partial C_{n}} \frac{\partial \Delta \mathcal{L}}{\partial \overrightarrow{\boldsymbol{r}}}-\frac{\partial \overrightarrow{\boldsymbol{f}}}{\partial C_{n}} \frac{d}{d t}\left(\overrightarrow{\boldsymbol{\Phi}}+\frac{\partial \Delta \mathcal{L}}{\partial \dot{\overrightarrow{\mathbf{r}}}}\right)-\frac{\partial \overrightarrow{\mathbf{g}}}{\partial C_{n}} \overrightarrow{\mathbf{\Phi}}
$$
}




$$
\begin{aligned}
& \sum_{j}\left(\left[C_{n} C_{j}\right]+\frac{\partial \overrightarrow{\boldsymbol{f}}}{\partial C_{n}} \frac{\partial}{\partial C_{j}}\left(\frac{\partial \Delta \mathcal{L}}{\partial \dot{\overrightarrow{\mathbf{r}}}}+\overrightarrow{\boldsymbol{\Phi}}\right)\right) \frac{d C_{j}}{d t}= \\
& \frac{\partial}{\partial C_{n}}\left[\Delta \mathcal{L}+\frac{1}{2}\left(\frac{\partial \Delta \mathcal{L}}{\partial \dot{\overrightarrow{\mathbf{r}}}}\right)^{2}\right]-\left(\frac{\partial \overrightarrow{\mathbf{g}}}{\partial C_{n}}+\frac{\partial \overrightarrow{\boldsymbol{f}}}{\partial C_{n}} \frac{\partial}{\partial t}+\frac{\partial \Delta \mathcal{L}}{\partial \dot{\overrightarrow{\mathbf{r}}}} \frac{\partial}{\partial C_{n}}\right)\left(\overrightarrow{\boldsymbol{\Phi}}+\frac{\partial \Delta \mathcal{L}}{\partial \dot{\overrightarrow{\mathbf{r}}}}\right),
\end{aligned}
$$

not only reveals the convenience of the generalised Lagrange gauge

$$
\overrightarrow{\boldsymbol{\Phi}}=-\frac{\partial \Delta \mathcal{L}}{\partial \dot{\overrightarrow{\mathbf{r}}}}
$$

(which reduces to $\overrightarrow{\boldsymbol{\Phi}}=0$ in the case of velocity-independent perturbations), but also explicitly demonstrates how the Hamiltonian variation comes into play: it is easy to notice that the sum in square brackets is equal to $-\Delta \mathcal{H}^{(\text {cont })}$. Substitution of the general-type force $\Delta \overrightarrow{\mathbf{F}}$ into (11) or of the Lagrangian perturbation $\Delta L$ into (17) gives us the gauge-invariant planetary equations, provided we know the expressions for elements of the inverse to the Lagrange-bracket matrix $\left[C_{i} C_{j}\right]$. When the elements $C_{i}$ are chosen as the Kepler or Delaunay variables, (17) entails the gauge-invariant versions of the Lagrange and Delaunay planetary equations (See Appendix 1 to Efroimsky \& Goldreich (2003).)

\subsection{Goldreich (1965)}

The earliest attempts to describe satellite motion about the precessing and nutating Earth were undertaken by Brouwer (1959), Proskurin \& Batrakov (1960), and Kozai (1960).

In 1965 Goldreich accomplished a ground-breaking work that marked the beginning of studies of the Martian satellite dynamics. He started out with two major assertions. One was that the Martian satellites had either been formed in the equatorial plane or been brought therein very long ago. The second was that Mars has experienced, though its history, a uniform precession. While the former proved to be almost certainly correct, 2 the validity of the latter remains model-dependent. While in the simplest approximation the planetary

If the disturbance were to depend upon positions solely, the first term on the right-hand side of the above equation would be equal simply to $\partial \Delta L / \partial C_{n}$. In general, though, we should rely on the chain rule

$$
\frac{\partial \Delta \mathcal{L}}{\partial C_{n}}=\frac{\partial \Delta \mathcal{L}}{\partial \overrightarrow{\boldsymbol{r}}} \frac{\partial \overrightarrow{\boldsymbol{f}}}{\partial C_{n}}+\frac{\partial \Delta \mathcal{L}}{\partial \dot{\overrightarrow{\boldsymbol{r}}}} \frac{\partial \dot{\overrightarrow{\mathbf{r}}}}{\partial C_{n}}=\frac{\partial \Delta \mathcal{L}}{\partial \overrightarrow{\boldsymbol{r}}} \frac{\partial \overrightarrow{\boldsymbol{f}}}{\partial C_{n}}+\frac{\partial \Delta \mathcal{L}}{\partial \dot{\overrightarrow{\boldsymbol{r}}}} \frac{\partial(\overrightarrow{\mathrm{g}}+\overrightarrow{\mathbf{\Phi}})}{\partial C_{n}},
$$

insertion whereof into the preceding formula entails:

$$
\sum_{j}\left[C_{n} C_{j}\right] \frac{d C_{j}}{d t}=\frac{\partial \Delta \mathcal{L}}{\partial C_{n}}-\frac{\partial \Delta \mathcal{L}}{\partial \dot{\overrightarrow{\boldsymbol{r}}}} \frac{\partial \overrightarrow{\boldsymbol{\Phi}}}{\partial C_{n}}-\left(\frac{\partial \overrightarrow{\boldsymbol{f}}}{\partial C_{n}} \frac{d}{d t}-\frac{\partial \overrightarrow{\mathbf{g}}}{\partial C_{n}}\right)\left(\overrightarrow{\boldsymbol{\Phi}}+\frac{\partial \Delta \mathcal{L}}{\partial \dot{\overrightarrow{\mathbf{r}}}}\right)
$$

To arrive herefrom to (17), one will have to add and subtract $(1 / 2) \partial(\partial(\Delta \mathcal{L}) / \partial \dot{\overrightarrow{\mathbf{r}}}) / \partial C_{n}$ on the right-hand side. Thereby one makes the gauge function $\overrightarrow{\boldsymbol{\Phi}}$ appear everywhere in the company of $\partial(\Delta \mathcal{L}) / \partial \dot{\overrightarrow{\mathbf{r}}}$.

${ }^{2}$ Phobos and Deimos give every appearance of being captured asteroids of the carbonaceous chondritic type (Veverka 1977, Pang et al. 1978, Pollack et al. 1979, Tolson et al. 1978). Given the considerations summarised in detail by Murison (1988), it seems least unlikely that the Martian satellites were formerly asteroids that were captured by the mechanism of gas drag. If so, this must have occurred early in the 
precession is uniform, a more involved analysis, carried out by Ward (1973, 1974), Laskar \& Robutel (1993), and Touma \& Wisdom (1994), offers evidence of strongly nonuniform, perhaps even chaotic, variations of the Martian obliquity at long time scales. It should be said, though, that the analysis presented by these authors was model-dependent. In particular, it was performed in the approximation of the planet being a nondissipative rigid rotator 3 Since the orbits of both satellites are located within less that $2^{o}$ from the equator, the two said assertions come to contradiction, unless there exists a mechanism constraining satellite orbits within the vicinity of the primary's equator. (Otherwise, as Goldreich noted in his paper, "the present low inclinations of these satellites' orbits would amount to an unbelievable coincidence.") In quest of such a mechanism, Goldreich (1965) investigated evolution of the Kepler elements of a satellite in a reference system co-precessing with the planet. He followed the traditional variation-of-parameters (VOP) scheme, i.e., assumed a two-body setting as an undisturbed problem and then treated the inertial forces, emerging in the co-precessing frame, as perturbation (along with another perturbation caused by the oblateness of the planet). Below we present a brief summary of Goldreich's results, with only minor comments.

Goldreich began by applying formulae (12-16) to motion in a coordinate system attached to the planet's centre of mass and precessing (but not spinning) with the planet. In this

history of the solar system while the gas disk was substantial enough to effect a capture. At that stage of planetary formation, the spin of the forming planet would be closely aligned with the orbital plane of the planet's motion about the Sun i.e., the obliquity would be small and the gas disk would be nearly coplanar with the planetary orbit. Energetically, a capture would be most likely to be equatorial. This is most easily seen in the context of the restricted three-body problem. The surfaces of zero velocity constrain any reasonable capture to occur from directions near the inner and outer collinear Lagrange points (Szebehely 1967, Murison 1988), which lie in the equatorial plane. Also, a somewhat inclined capture would quickly be equatorialised by the gas disk. If the capture inclination is too high, the orbital energy is then too high to allow a long enough temporary capture, and the object would hence not encounter enough drag over a long enough time to effect a permanent capture (Murison 1988). Thus, Phobos and Deimos were likely (in as much as we can even use that term) to have been captured into near-equatorial orbits.

${ }^{3}$ In all these studies the planet was modelled as a rigid body. Touma \& Wisdom (1994) emphasised in their research that they assumed Mars to be always in its principal rotation state. A common feature of all these models was their neglect of the inelastic relaxation of the spin axis' precession. Whether the latter simplification is physically justified is yet to be determined. Inelastic dissipation is, essentially, the internal friction and results from alternating stresses and strains emerging in a wobbling rotator (Prendergast 1958; Burns \& Safronov 1973; Efroimsky 2001, 2002c; Molina, Moreno \& Martinez-López 2003; Sharma, Burns \& Hui 2004). It is well-known that the lower the frequency of precession the slower dissipation of the elastic energy associated therewith. This circumstance suggests that slow obliquity changes will result in virtually no damping. (The same circumstance makes one think that the fastest modes of spin precession are well dissipated. It was for this reason that Touma \& Wisdom (1994) accepted the model of Mars always being in its principal-rotation state.) The real physical picture is far more complicated, mainly due to the essentially nonlinear nature of the phenomenon. Since Mars is not a perfectly symmetrical oblate spheroid but is a triaxial body, its free rotation in a spin state, which is even slightly different from the principal one, is described by the elliptic functions of Jacobi whose decomposition over sines and cosines contains an infinite multitude of harmonics. From here, it is possible to demonstrate that in the course of rotation a continuous exchange of energy among these harmonics is taking place. Due to this phenomenon, the alternating stresses associated with short-period precession are always getting energy from stresses caused by long-period changes of the spin axis. This way, dissipation of the short-time motions indirectly leads to damping of the long-period ones. This phenomenon, called "energy cascade" is very well known in the theory of turbulence, but in fact it is generic and shows itself in nonlinear situations. In the precessing-top context it has not been explored so far, and we do not know how effective it is in restraining the obliquity variations caused by solar torque. 
system, the equation of motion includes inertial forces and, therefore, reads:

$$
\begin{gathered}
\ddot{\overrightarrow{\mathbf{r}}}=-\frac{\partial U}{\partial \overrightarrow{\mathbf{r}}}-2 \overrightarrow{\boldsymbol{\mu}} \times \dot{\overrightarrow{\mathbf{r}}}-\dot{\overrightarrow{\boldsymbol{\mu}}} \times \overrightarrow{\mathbf{r}}-\overrightarrow{\boldsymbol{\mu}} \times(\overrightarrow{\boldsymbol{\mu}} \times \overrightarrow{\mathbf{r}})= \\
-\frac{\partial U_{o}}{\partial \overrightarrow{\mathbf{r}}}-\frac{\partial \Delta U}{\partial \overrightarrow{\mathbf{r}}}-2 \overrightarrow{\boldsymbol{\mu}} \times \dot{\overrightarrow{\mathbf{r}}}-\dot{\vec{\mu}} \times \overrightarrow{\mathbf{r}}-\overrightarrow{\boldsymbol{\mu}} \times(\overrightarrow{\boldsymbol{\mu}} \times \overrightarrow{\mathbf{r}}),
\end{gathered}
$$

dots denoting time derivatives in the co-precessing frame and $\overrightarrow{\boldsymbol{\mu}}$ standing for the coordinate system angular velocity relative to an inertial frame 4 Here the physical (i.e., not associated with inertial forces) potential $U(\overrightarrow{\boldsymbol{r}})$ consists of the (reduced) two-body part $U_{o}(\overrightarrow{\boldsymbol{r}}) \equiv-G M \overrightarrow{\boldsymbol{r}} / r^{3}$ and a term $\Delta U(\overrightarrow{\boldsymbol{r}})$ caused by the planet's oblateness. The overall disturbing force on the right-hand side of the above equation is generated, according to (16), by

$$
\Delta \mathcal{L}(\overrightarrow{\mathbf{r}}, \dot{\overrightarrow{\mathbf{r}}}, t)=-\Delta U(\overrightarrow{\mathbf{r}})+\dot{\overrightarrow{\mathbf{r}}} \cdot(\overrightarrow{\boldsymbol{\mu}} \times \overrightarrow{\mathbf{r}})+\frac{1}{2}(\overrightarrow{\boldsymbol{\mu}} \times \overrightarrow{\mathbf{r}}) \cdot(\overrightarrow{\boldsymbol{\mu}} \times \overrightarrow{\mathbf{r}})
$$

Since in this case

$$
\frac{\partial \Delta \mathcal{L}}{\partial \dot{\mathbf{r}}}=\overrightarrow{\boldsymbol{\mu}} \times \overrightarrow{\mathbf{r}}
$$

then

$$
\overrightarrow{\mathbf{p}}=\dot{\overrightarrow{\mathbf{r}}}+\frac{\partial \Delta \mathcal{L}}{\partial \dot{\overrightarrow{\mathbf{r}}}}=\dot{\overrightarrow{\mathbf{r}}}+\overrightarrow{\boldsymbol{\mu}} \times \overrightarrow{\mathbf{r}}
$$

and, therefore, the appropriate Hamiltonian variation will look:

$$
\begin{gathered}
\Delta \mathcal{H}=-\left[\Delta \mathcal{L}+\frac{1}{2}\left(\frac{\partial \Delta \mathcal{L}}{\partial \dot{\mathbf{r}}}\right)^{2}\right]= \\
-[-\Delta U+\overrightarrow{\mathbf{p}} \cdot(\overrightarrow{\boldsymbol{\mu}} \times \overrightarrow{\mathbf{r}})]=\Delta U-(\overrightarrow{\mathbf{r}} \times \overrightarrow{\mathbf{p}}) \cdot \overrightarrow{\boldsymbol{\mu}}=\Delta U-\overrightarrow{\mathbf{J}} \cdot \overrightarrow{\boldsymbol{\mu}} .
\end{gathered}
$$

This way, $\Delta \mathcal{H}$ becomes expressed through quantities defined in the co-precessing frame: the satellite's orbital momentum vector $\overrightarrow{\mathbf{J}}=\overrightarrow{\mathbf{r}} \times \overrightarrow{\mathbf{p}}$ and the precession rate $\overrightarrow{\boldsymbol{\mu}}$.

Goldreich employed the above expression in the role of a disturbing function $R$ in the planetary equations:

$$
\begin{aligned}
\frac{d i}{d t} & =\frac{\cos i}{n a^{2}\left(1-e^{2}\right)^{1 / 2} \sin i} \frac{\partial(-\Delta \mathcal{H})}{\partial \omega}-\frac{1}{n a^{2}\left(1-e^{2}\right)^{1 / 2} \sin i} \frac{\partial(-\Delta \mathcal{H})}{\partial \Omega} \\
\frac{d \Omega}{d t} & =\frac{1}{n a^{2}\left(1-e^{2}\right)^{1 / 2} \sin i} \frac{\partial(-\Delta \mathcal{H})}{\partial i}
\end{aligned}
$$

${ }^{4}$ Be mindful that $\overrightarrow{\boldsymbol{\mu}}$, though being a precession rate relative to an inertial frame, is a vector defined in the co-precessing frame. (For details see section 8.6 in Marsden and Ratiu (2003) or section 27 in Arnold (1989).) In this frame,

$$
\overrightarrow{\boldsymbol{\mu}}=\hat{\mathbf{x}}_{1} \frac{d i_{p}}{d t}+\hat{\mathbf{x}}_{2} \frac{d h_{p}}{d t} \sin i_{p}+\hat{\mathbf{x}}_{3} \frac{d h_{p}}{d t} \cos i_{p}
$$

$i_{p}$ and $h_{p}$ being the inclination and the longitude of the node of the planetary equator of date relative to that of epoch. 
where

$$
-\Delta \mathcal{H} \equiv R=R_{\text {oblate }}+R_{\text {inertial }}
$$

consists, according to (22), of two inputs:5

$$
R_{\text {oblate }}(\nu) \equiv-\Delta U=\frac{G m J_{2}}{2} \frac{\rho^{2}}{r^{3}}\left[1-3 \sin ^{2} i \sin ^{2}(\omega+\nu)\right],
$$

and

$$
R_{\text {inertial }} \equiv \overrightarrow{\mathbf{J}} \cdot \overrightarrow{\boldsymbol{\mu}}=\sqrt{G m a\left(1-e^{2}\right)} \overrightarrow{\boldsymbol{w}} \cdot \overrightarrow{\boldsymbol{\mu}}
$$

Here $m \equiv\left(m_{\text {primary }}+m_{\text {secondary }}\right)$. The mean motion is, as ever, $n \equiv(G m)^{1 / 2} a^{-3 / 2}$, while $\rho$ stands for the mean radius of the primary, $\nu$ denotes the true anomaly, and

$$
r=a \frac{1-e^{2}}{1+e \cos \nu}
$$

is the instantaneous orbital radius. In the right-hand side of (27) it was assumed that the angular momentum is connected with the orbital elements through the well-known formula

$$
\overrightarrow{\mathbf{J}} \equiv \overrightarrow{\boldsymbol{r}} \times \overrightarrow{\boldsymbol{p}}=\sqrt{G m a\left(1-e^{2}\right)} \quad \overrightarrow{\boldsymbol{w}}
$$

where

$$
\overrightarrow{\boldsymbol{w}}=\hat{\mathbf{x}}_{1} \sin i \sin \Omega-\hat{\mathbf{x}}_{2} \sin i \cos \Omega+\hat{\mathbf{x}}_{3} \cos i
$$

is a unit vector normal to the instantaneous ellipse, expressed through unit vectors $\hat{\mathbf{x}}_{1}, \hat{\mathbf{x}}_{2}, \hat{\mathbf{x}}_{3}$ associated with the co-precessing frame $x_{1}, x_{2}, x_{3}$ (the axes $x_{1}$ and $x_{2}$ lying in the planet's equatorial plane). The afore-written expression for $\overrightarrow{\boldsymbol{w}}$ evidently yields:

$$
R_{\text {inertial }}=\sqrt{G m a\left(1-e^{2}\right)}\left(\mu_{1} \sin i \sin \Omega-\mu_{2} \sin i \cos \Omega+\mu_{3} \cos i\right),
$$

while (26) may be, in the first approximation, substituted with its secular part, i.e., with its average over the orbital period:

$$
\left\langle R_{\text {oblate }}\right\rangle=\frac{n^{2} J_{2}}{4} \rho^{2} \frac{3 \cos ^{2} i-1}{\left(1-e^{2}\right)^{3 / 2}},
$$

the averaging having been carried out through the medium of formula (109) from the Appendix, with (28) inserted. With the aid of (30) and (31), the planetary equations (24) and (25) will simplify to:

$$
\frac{d i}{d t}=-\mu_{1} \cos \Omega-\mu_{2} \sin \Omega
$$

${ }^{5}$ Our formula (26) slightly differs from the one employed by Goldreich (1965), because here we use the modern definition of $J_{2}$ :

$$
U=-\frac{\mu}{r}\left\{1-\sum_{m=2}^{\infty} J_{m}\left(\frac{\rho}{r}\right)^{m} P_{m}(\sin \alpha)\right\},
$$

$\alpha$ being the satellite's latitude in the planet-related coordinate system. The coefficient $J$ used by Goldreich (1965) differs from our $J_{2}$ by a constant factor: $J=(3 / 2) J_{2} \rho^{2} / r^{2}$. 


$$
\frac{d \Omega}{d t}=-\frac{3}{2} n J_{2}\left(\frac{\rho}{a}\right)^{2} \frac{\cos i}{\left(1-e^{2}\right)^{2}}+O\left(\frac{|\vec{\mu}|}{J_{2} n}\right)
$$

The latter results in the well-known node-precession formula,

$$
\Omega=\Omega_{o}-\frac{3}{2} n J_{2}\left(\frac{\rho}{a}\right)^{2} \frac{\cos i}{\left(1-e^{2}\right)^{2}}\left(t-t_{o}\right) .
$$

Its insertion into the former entails

$$
i=-\frac{\mu_{1}}{\chi} \cos \left[-\chi\left(t-t_{o}\right)+\Omega_{o}\right]+\frac{\mu_{2}}{\nu} \sin \left[-\chi\left(t-t_{o}\right)+\Omega_{o}\right]+i_{o},
$$

where

$$
\chi \equiv \frac{3}{2} n J_{2}\left(\frac{\rho}{a}\right)^{2} \frac{\cos i}{\left(1-e^{2}\right)^{2}} .
$$

In Goldreich (1965), equation (35) was the main result, its derivation being valid for wobble which is slow $\left(|\overrightarrow{\boldsymbol{\mu}}| \ll J_{2} n\right)$ and close to uniform $\left(|\dot{\overrightarrow{\boldsymbol{\mu}}}| /|\overrightarrow{\boldsymbol{\mu}}| \ll J_{2} n\right)$.

Despite a warning issued by Goldreich in his paper, this result has often been misinterpreted and, therefore, misused in publications devoted to satellites and rings of wobbling planets, as well as in the literature on orbits about tumbling galaxies.

In (35) $i$ stands for the inclination defined in co-precessing axes associated with the planet's equator, and therefore (35) clearly demonstrates that, in the course of obliquity changes, this inclination oscillates about zero, with no secular shift accumulated. Does this necessarily mean that the satellite orbit, too, oscillates about the equatorial plane, without a secular deviation therefrom? Most surprisingly, the answer to this question is negative. The reason for this is that so calculated orbital elements, though defined in the co-precessing frame, are not osculating therein. In other words, in the frame where the elements are introduced, the instantaneous ellipses parameterised by these elements are not tangent to the physical orbit as seen in this frame.

This circumstance was emphasised by Goldreich, who noticed that formula (29) normally (i.e., when employed in an inertial frame) connects the osculating elements defined in that frame with the angular momentum $\overrightarrow{\boldsymbol{r}} \times \overrightarrow{\boldsymbol{p}}$ defined in the same, inertial, frame (i.e., with $\overrightarrow{\boldsymbol{r}} \times \dot{\overrightarrow{\boldsymbol{r}}}$ ). Since in the above calculation the frame is not inertial (and, therefore, the angular momentum is different from $\overrightarrow{\boldsymbol{r}} \times \dot{\overrightarrow{\boldsymbol{r}}}$ but is equal to $\overrightarrow{\boldsymbol{r}} \times \overrightarrow{\boldsymbol{p}}=\overrightarrow{\boldsymbol{r}} \times(\dot{\overrightarrow{\boldsymbol{r}}}+\overrightarrow{\boldsymbol{\mu}} \times \overrightarrow{\boldsymbol{r}}))$, then the orbital elements returned by (29) cannot be osculating in this frame 6 On these grounds Goldreich warned the reader of the peculiar nature of the elements used in his integration.

To this we would add that it is not at all evident that the inertial-forces-caused alteration of the planetary equations should be achieved through amending the disturbing function with

${ }^{6}$ Were these elements osculating in the frame wherein they had been defined, then formula (29) would read: $\overrightarrow{\boldsymbol{r}} \times \dot{\overrightarrow{\boldsymbol{r}}}=\sqrt{G m a\left(1-e^{2}\right)} \overrightarrow{\boldsymbol{w}}$, i.e., would connect the elements with the velocity in that frame. In reality, though, it reads: $\overrightarrow{\boldsymbol{r}} \times \overrightarrow{\boldsymbol{p}}=\sqrt{G m a\left(1-e^{2}\right)} \overrightarrow{\boldsymbol{w}}$, i.e., connects the elements with the momentum $\overrightarrow{\boldsymbol{p}}=\dot{\overrightarrow{\boldsymbol{r}}}+\overrightarrow{\boldsymbol{\mu}} \times \overrightarrow{\boldsymbol{r}}$ which happens to coincide with the satellite's velocity relative to the inertial axes. This situation was formulated by Goldreich in the following terms: the orbital elements emerging in the above derivation are defined in the co-precessing frame, but are osculating in the inertial one. This illustrative metaphor should not, though, be overplayed: the fact that the elements emerging in Goldreich's computation return the inertial-frame-related velocity does not mean that this inclination may be interpreted as that relative to the invariable plane. (The elements were introduced in the co-precessing frame!) 
the momentum-dependent variation of the negative Hamiltonian, $\overrightarrow{\mathbf{J}} \cdot \overrightarrow{\boldsymbol{\mu}}$. While the common fallacy identifies the disturbing function with the negative Hamiltonian perturbation, in reality this rule-of-thumb works (and yields elements that are osculating) only for disturbances dependent solely upon positions, not upon velocities (i.e., for Hamiltonian perturbations dependent only upon coordinates, but not upon momenta). Ours is not that case and, therefore, more alterations in the planetary equations are needed to account for the frame precession, if we wish these equations to render osculating elements. However, if one neglects this circumstance and simply amends the disturbing function with $\overrightarrow{\mathbf{J}} \cdot \overrightarrow{\boldsymbol{\mu}}$, then the planetary equations will give some elements different from the osculating ones. It will then become an interesting question as to whether such elements will or will not coincide with those rendered by (28) when this formula is used in non-inertial frames.

All these subtle issues get untangled in the framework of the gauge formalism. Application of this formalism to motions in non-inertial frames of references was presented in Efroimsky \& Goldreich (2004). The main results proven there are the following.

1. If one attempts to account for the inertial forces by simply adding the term $\overrightarrow{\mathbf{J}} \cdot \overrightarrow{\boldsymbol{\mu}}$ to the disturbing function, with no other alterations made in the planetary equations, then these equations indeed do render quantities that may be interpreted as some orbital elements (i.e., as parameters of some instantaneous conics). These elements are NOT osculating and, therefore, the instantaneous conics parameterised by there elements are not tangent to the physical orbit. Hence, these elements cannot, generally, be attributed a direct physical interpretation $\sqrt[7]{ }$ except in situations where their deviation from the osculating elements remains sufficiently small.

2. By a remarkable coincidence, these non-osculating elements turned out to be identical with those emerging in formula (29). This coincidence was implicitly taken for granted by Goldreich (1965), which reveals his truly incredible scientific intuition.

3. To build up a system of planetary equations that render osculating elements of the orbit as seen in the co-precessing coordinate system, one has not only to add $\overrightarrow{\mathbf{J}} \cdot \overrightarrow{\boldsymbol{\mu}}$ to the disturbing function, but also to amend each of these equations with several extra terms. Some of those terms are of order $\left(|\overrightarrow{\boldsymbol{\mu}}| /\left(J_{2} n\right)\right)^{2}$; some others are of order $|\dot{\overrightarrow{\boldsymbol{\mu}}}| /\left(|\overrightarrow{\boldsymbol{\mu}}| J_{2} n\right)$. Most importantly, some terms are of first order in the precession-caused perturbation $|\overrightarrow{\boldsymbol{\mu}}| /\left(J_{2} n\right)$, which means right away that the non-osculating elements used by Goldreich (1965) differ from the osculating ones already in the first order. While a more comprehensive account on this topic, with the resulting equations, will be offered at the end of this section, here we shall touch upon only one question which gets immediately raised by the presence of such first-order differences. This question is: what are the averages of these differences? Stated alternatively, do the secular components of the said non-osculating elements differ considerably from those of the osculating ones? Goldreich (1965) stated, without proof, that the secular components differ only in high orders over the velocity-dependent part of the perturbation. In our paper we shall probe the limits for this assumption.

\footnotetext{
${ }^{7}$ When the orbit evolution is sufficiently slow, the observer can attribute some physical meaning to elements of the osculating conic. For example, whenever an observer talks about the inclination or the eccentricity of a perturbed orbit, he naturally implies those of the osculating ellipse or hyperbola.
} 


\subsection{Brumberg and Kinoshita}

A development, part of which was similar to that of Goldreich (1965), was independently carried out by Brumberg, Evdokimova \& Kochina (1971), who studied orbits of artificial lunar satellites in a coordinate system co-precessing with the Moon. In that article, too, the non-osculating nature of the resulting orbital variables did not go unnoticed. The authors called these variables "contact elements" and stated (though never proved) that these variables return not the correct value of the velocity but that of the momentum. Later, one of these authors rightly noted in his book (Brumberg 1992) that the contact elements differ from the osculating ones already in the first order over the velocity-dependent part of the perturbation. In subsection 1.1 .3 of that book, he unsuccessfully tried to derive analytical transformations interconnecting these sets of variables 8

A similar attempt was undertaken in a very interesting article by Ashby \& Allison (1993). Though the authors succeeded in many other points, their attempt to derive formulae for such a gauge transformation was not successful.9.9

The setting, considered by Goldreich (1965) in the context of Martian satellites and by Brumberg et al (1971) in the context of circumlunar orbits, later emerged in the article by Kinoshita (1993), who addressed the satellites of Uranus.

Kinoshita's treatment of the problem was based on the following mathematical construction. Denote satellite positions and velocities in the inertial and in the co-precessing axes with $\left\{\overrightarrow{\boldsymbol{r}}^{\prime}, \overrightarrow{\mathbf{v}}^{\prime}\right\}$ and with $\{\overrightarrow{\boldsymbol{r}}, \overrightarrow{\mathbf{v}}\}$, correspondingly 10 Interconnection between them will be implemented by an orthogonal matrix $\hat{A}$,

$$
\overrightarrow{\boldsymbol{r}}=\hat{A} \overrightarrow{\boldsymbol{r}}^{\prime}, \quad \overrightarrow{\mathbf{v}} \equiv \dot{\overrightarrow{\boldsymbol{r}}}=\dot{\hat{A}} \overrightarrow{\boldsymbol{r}}^{\prime}+\hat{A} \dot{\overrightarrow{\boldsymbol{r}}}^{\prime}=\dot{\hat{A}} \hat{A}^{-1} \overrightarrow{\boldsymbol{r}}+\hat{A} \overrightarrow{\mathbf{v}}^{\prime}=-\overrightarrow{\boldsymbol{\mu}} \times \overrightarrow{\boldsymbol{r}}+\hat{A} \overrightarrow{\mathbf{p}}^{\prime},
$$

$\overrightarrow{\boldsymbol{\mu}}$ being the precession rate as seen in the co-precessing coordinate system, and the inertial velocity $\overrightarrow{\mathbf{v}}^{\prime}$ being identical to the inertial momentum $\overrightarrow{\mathbf{p}}^{\prime}$. Kinoshita suggested interpreting this interconnection as a canonical transformation between variables $\left\{\overrightarrow{\boldsymbol{r}}^{\prime}, \overrightarrow{\boldsymbol{p}}^{\prime}\right\}$ and $\{\overrightarrow{\boldsymbol{r}}, \overrightarrow{\boldsymbol{p}}\}$, implemented by generating function

$$
F_{2}=\overrightarrow{\boldsymbol{p}} \cdot \hat{A} \overrightarrow{\boldsymbol{r}}^{\prime}=\left(\hat{A}^{T} \overrightarrow{\boldsymbol{p}}\right) \cdot \overrightarrow{\boldsymbol{r}}^{\prime} .
$$

${ }^{8}$ Contrary to the author's statement, formula (1.1.41) in Brumberg (1992) is not rigorous, but is valid only to first order. (To make it rigorous, one should substitute everywhere, except in the denominators, $\dot{\vec{r}}$ with $\dot{\overrightarrow{\boldsymbol{r}}}-\partial R / \partial \dot{\overrightarrow{\boldsymbol{r}}}$.) Besides, the author did not demonstrate his derivation of formula (1.1.43), for $M_{o}$, from (1.1.42). (In Brumberg's book the mean anomaly is denoted with $l$, not with $M$. )

Most importantly, the qualitative reasoning presented by the author in the paragraph preceding formula (1.1.43) is unrigorous and essentially incorrect. The cause of this is that the author compares the planetary equations for contact elements, written in a precessing frame, with the equations for osculating ones, written in an inertial frame, instead of comparing two such systems (for contact and for osculating elements), both of which are written in a precessing frame. This makes a big difference because, as we already explained above, transition to a precessing frame does not simply mean addition of an extra term to the disturbing function.

Despite all these mathematical irregularities, the averaged system of planetary equations (1.1.44), "derived" by Brumberg for the first-order secular perturbations, turns out to be correct in the limit of uniform precession. Just as in the preceding subsection we had a reason to praise the unusual intuition of Goldreich, so here we have to pay tribute to the excellent intuition of Brumberg, intuition which superseded his flawed mathematics.

${ }^{9}$ To carry out the gauge transformation, the authors used a set of intermediate variables $\left\{Q_{(o)}^{k}, P_{k(o)}\right\}$, which were canonical and, at the same time, osculating. As follows from the theorem proven by Efroimsky \& Goldreich (2003), these variables are nonexistent when the perturbation depends upon velocities.

${ }^{10}$ We use notations opposite to those in Kinoshita (1993), in order to conform with the notations of Goldreich (1965). 
This choice of generating function rightly yields

$$
\overrightarrow{\boldsymbol{r}}=\frac{\partial F_{2}}{\partial \overrightarrow{\boldsymbol{p}}}=\hat{A} \overrightarrow{\boldsymbol{r}}^{\prime}
$$

while the interconnection between momenta will look:

$$
\overrightarrow{\boldsymbol{p}}^{\prime}=\frac{\partial F_{2}}{\partial \overrightarrow{\boldsymbol{r}}^{\prime}}=\hat{A}^{T} \overrightarrow{\boldsymbol{p}}, \text { i.e., } \quad \overrightarrow{\boldsymbol{p}}=\left(\hat{A}^{T}\right)^{-1} \overrightarrow{\boldsymbol{p}}^{\prime}=\hat{A} \overrightarrow{\boldsymbol{p}}^{\prime}
$$

whence $\overrightarrow{\boldsymbol{p}}=\overrightarrow{\mathbf{v}}+\overrightarrow{\boldsymbol{\mu}} \times \overrightarrow{\boldsymbol{r}}$. The Hamiltonian in precessing axes will read

$$
\begin{gathered}
H(\overrightarrow{\boldsymbol{r}}, \overrightarrow{\boldsymbol{p}})=H^{(\text {inert })}\left(\overrightarrow{\boldsymbol{r}}^{\prime}, \overrightarrow{\boldsymbol{p}}^{\prime}\right)+\frac{\partial F_{2}}{\partial t}=H^{(\text {inert })}\left(\overrightarrow{\boldsymbol{r}}^{\prime}, \overrightarrow{\boldsymbol{p}}^{\prime}\right)+\overrightarrow{\boldsymbol{p}} \cdot \dot{\hat{A}} \overrightarrow{\boldsymbol{r}}^{\prime} \\
=H^{(\text {inert })}\left(\overrightarrow{\boldsymbol{r}}^{\prime}, \overrightarrow{\boldsymbol{p}}^{\prime}\right)-\overrightarrow{\boldsymbol{p}} \cdot(\overrightarrow{\boldsymbol{\mu}} \times \overrightarrow{\boldsymbol{r}})=H^{(\text {inert })}\left(\overrightarrow{\boldsymbol{r}}^{\prime}, \overrightarrow{\boldsymbol{p}}^{\prime}\right)-(\overrightarrow{\boldsymbol{r}} \times \overrightarrow{\boldsymbol{p}}) \cdot \overrightarrow{\boldsymbol{\mu}} .
\end{gathered}
$$

The Hamiltonian perturbation, caused by the inertial forces, is $-(\overrightarrow{\boldsymbol{r}} \times \overrightarrow{\boldsymbol{p}}) \cdot \overrightarrow{\boldsymbol{\mu}}=-\overrightarrow{\mathbf{J}} \times \overrightarrow{\boldsymbol{\mu}}$, vector $\overrightarrow{\mathbf{J}}$ being the orbital angular momentum as seen in the co-precessing frame. Comparing this with (22), we see that employment of the above canonical transformation is but another method of stepping on the same rake. In distinction from Goldreich (1965) and Brumberg et al (1971), Kinoshita in his paper did not notice that he was working with non-osculating elements.

The problem with Kinoshita's treatment is that the condition of canonicity in some situations comes into contradiction with the osculation condition. In other words, canonicity sometimes implicitly contains a constraint that sometimes is different from the Lagrange constraint (5). This issue was comprehensively elucidated in the work Efroimsky \& Goldreich (2003). The authors began with the reduced two-body setting and thoroughly re-examined the Hamilton-Jacobi procedure, which leads one from the spherical coordinates and the corresponding canonical momenta to the set of Delaunay variables. While in the undisturbed two-body case this procedure yields the Delaunay variables which are trivially osculating (and parameterise a fixed Keplerian ellipse or hyperbola), in the perturbed case the situation becomes more involved. According to the theorem proven in that paper, the resulting Delaunay elements are osculating (and parameterise a conic tangent to the perturbed trajectory) if the Hamiltonian perturbation depends solely upon positions, not upon momenta (or, the same: if the Lagrangian perturbation depends upon positions but not upon velocities). Otherwise, the Delaunay elements turn out to be non-osculating (and parametrise the physical trajectory with a sequence of non-tangent conics). As one can see from the above equation, the Hamiltonian perturbation, caused by the inertial forces, depends upon the momentum, and this circumstance indicates the problem. This trap, in which many have fallen, is of special importance in General Relativity, because the relativistic corrections to the equations of motion are velocity-dependent 11

\footnotetext{
${ }^{11}$ In an interesting article (Chernoivan \& Mamaev 1999), the authors addressed the two-body problem on a curved background. The curvature entailed a velocity-dependent relativist correction, which was treated as a perturbation. After carrying out the Hamilton-Jacobi development, the authors arrived at canonical variables analogous to the Delaunay elements. Orbit integration in terms of these variables would be as correct as in terms of any others. The problems began when the authors used these elements to come to some conclusions regarding the perihelion precession. Those conclusions need to be reconsidered, because they were rendered on the basis of Delaunay elements that were non-osculating. Similar comments may be made about the work by Richardson \& Kelly (1988) who addressed, using the Hamiltonian formalism, the relativist two-body problem in the post-Newtonian approximation.
} 


\section{Planetary equations}

In this section we shall briefly spell out some results obtained in Efroimsky \& Goldreich (2004) and shall use these results to derive the Lagrange-type planetary equations (59 - 64) for osculating elements in a coordinate system co-precessing with an oblate primary.

\subsection{Planetary equations for contact elements}

Above, in subsection 2.1, we provided a very short account of the gauge formalism. Expression (17), presented there, is the most general form of the planetary equations for an arbitrary set of six independent orbital elements, written in terms of an arbitrary disturbance of the Lagrangian.

When the elements $C_{i}$ are chosen to be the Keplerian or Delaunay sets of variables, we arrive at the gauge-invariant versions of the Lagrange or Delaunay planetary equations, correspondingly. They are written down in the Appendix to Efroimsky \& Goldreich (2003). Interplay between the gauge freedom and the freedom of frame choice is explained at length in Section 3 of the article Efroimsky \& Goldreich (2004), which addresses orbits about a precessing planet. It is demonstrated in that work that, if one chooses to describe the motion in terms of the non-osculating elements that were introduced in a co-precessing frame and defined in the generalised Lagrange gauge 12 (18), then the corresponding Hamiltonian perturbation will read:

$$
\Delta \mathcal{H}^{(\text {cont })}=-\left[R_{\text {oblate }}(\overrightarrow{\boldsymbol{f}})+\overrightarrow{\boldsymbol{\mu}} \cdot(\overrightarrow{\boldsymbol{f}} \times \overrightarrow{\mathrm{g}})\right],
$$

while the planetary equations (17) acquire the form

$$
\left[C_{r} C_{i}\right] \frac{d C_{i}}{d t}=\frac{\partial\left(-\Delta \mathcal{H}^{(\text {cont })}\right)}{\partial C_{r}},
$$

or:

$$
\left[C_{r} C_{i}\right] \frac{d C_{i}}{d t}=\frac{\partial}{\partial C_{r}}\left[R_{\text {oblate }}(\overrightarrow{\boldsymbol{f}})+\overrightarrow{\boldsymbol{\mu}} \cdot(\overrightarrow{\boldsymbol{f}} \times \overrightarrow{\mathbf{g}})\right]
$$

where $\overrightarrow{\boldsymbol{f}}$ and $\overrightarrow{\mathrm{g}}$ stand for the undisturbed (two-body) functional expressions of the position and velocity via the time and the chosen set of orbital elements:

$$
\begin{aligned}
\overrightarrow{\mathbf{r}} & =\overrightarrow{\boldsymbol{f}}\left(C_{1}, \ldots, C_{6}, t\right) \\
\overrightarrow{\mathbf{v}} & =\overrightarrow{\mathbf{g}}\left(C_{1}, \ldots, C_{6}, t\right) \equiv \frac{\partial}{\partial t} \overrightarrow{\boldsymbol{f}}\left(C_{1}, \ldots, C_{6}, t\right)
\end{aligned}
$$

(so that $\overrightarrow{\boldsymbol{f}}\left(C_{1}(t), \ldots, C_{6}(t), t\right)$ and $\overrightarrow{\mathrm{g}}\left(C_{1}(t), \ldots, C_{6}(t), t\right)$ become the ansatz for solving the disturbed problem). For $R_{\text {oblate }}$ in (42) - (43), one can employ, dependent upon the desired degree of rigour, either the exact expression (26) or its orbital average (31).

In the generalised Lagrange gauge (18) the canonical momentum becomes:

$$
\overrightarrow{\mathbf{p}}=\dot{\overrightarrow{\mathbf{r}}}+\frac{\partial \Delta \mathcal{L}}{\partial \dot{\overrightarrow{\mathbf{r}}}}=\overrightarrow{\mathbf{g}}+\overrightarrow{\mathbf{\Phi}}+\frac{\partial \Delta \mathcal{L}}{\partial \dot{\overrightarrow{\mathbf{r}}}}=\overrightarrow{\mathbf{g}}
$$

\footnotetext{
${ }^{12}$ It is an absolutely crucial point that choice of a gauge and choice of a reference frame are two totally independent procedures. In each frame one has an opportunity to choose among an infinite variety of gauges.
} 
which means that its functional dependence upon the time and the chosen set of orbital elements is the same as it used to be in the unperturbed case where both the velocity and the momentum were simply equal to $\overrightarrow{\mathrm{g}}\left(C_{1}, \ldots, C_{6}, t\right)$. This also means that $\Delta \mathcal{H}^{\text {(cont) }}$ coincides with Goldreich's $\Delta \mathcal{H}$ given by (26) $)$.

We see that the generalised Lagrange gauge (18) singles out the same set of non-osculating elements which showed up in the studies by Goldreich (1965) and Brumberg, Evdokimova \& Kochina (1971), - the set of "contact elements." This is why in (42) the Hamiltonian perturbation was written with the superscript "cont."

In (43) the Lagrange-bracket matrix is defined in the unperturbed two-body fashion (9) and can, therefore, be trivially inverted. Hence, when the elements are chosen as the Keplerian ones, the appropriate equations look like the customary Lagrange-type equations (i.e., like (24) and (25) above), with the disturbing function given by (26) or, equivalently, by (42):

$$
\begin{aligned}
& \frac{d a}{d t}=\frac{2}{n a} \frac{\partial\left(-\Delta \mathcal{H}^{(\text {cont })}\right)}{\partial M_{o}} \\
& \frac{d e}{d t}=\frac{1-e^{2}}{n a^{2} e} \frac{\partial\left(-\Delta \mathcal{H}^{(\text {cont })}\right)}{\partial M_{o}}-\frac{\left(1-e^{2}\right)^{1 / 2}}{n a^{2} e} \frac{\partial\left(-\Delta \mathcal{H}^{(\text {cont })}\right)}{\partial \omega}, \\
& \frac{d \omega}{d t}=\frac{-\cos i}{n a^{2}\left(1-e^{2}\right)^{1 / 2} \sin i} \frac{\partial\left(-\Delta \mathcal{H}^{(\text {cont })}\right)}{\partial i}+\frac{\left(1-e^{2}\right)^{1 / 2}}{n a^{2} e} \frac{\partial\left(-\Delta \mathcal{H}^{(\text {cont })}\right)}{\partial e} \\
& \frac{d i}{d t}=\frac{\cos i}{n a^{2}\left(1-e^{2}\right)^{1 / 2} \sin i} \frac{\partial\left(-\Delta \mathcal{H}^{(\text {cont })}\right)}{\partial \omega}-\frac{1}{n a^{2}\left(1-e^{2}\right)^{1 / 2} \sin i} \frac{\partial\left(-\Delta \mathcal{H}^{(\text {cont })}\right)}{\partial \Omega} \\
& \frac{d \Omega}{d t}=\frac{1}{n a^{2}\left(1-e^{2}\right)^{1 / 2} \sin i} \frac{\partial\left(-\Delta \mathcal{H}^{(\text {cont })}\right)}{\partial i} \\
& \frac{d M_{o}}{d t}=-\frac{1-e^{2}}{n a^{2} e} \frac{\partial\left(-\Delta \mathcal{H}^{(\text {cont })}\right)}{\partial e}-\frac{2}{n a} \frac{\partial\left(-\Delta \mathcal{H}^{(\text {cont })}\right)}{\partial a},
\end{aligned}
$$




\subsection{Planetary equations for osculating elements}

When one introduces elements in the precessing frame and also demands that they osculate in this frame (i.e., makes them obey the Lagrange gauge $\overrightarrow{\boldsymbol{\Phi}}=0$ ) then the Hamiltonian variation reads 13

$$
\Delta \mathcal{H}^{(o s c)}=-\left[R_{\text {oblate }}(\nu)+\overrightarrow{\boldsymbol{\mu}} \cdot(\overrightarrow{\boldsymbol{f}} \times \overrightarrow{\mathbf{g}})+(\overrightarrow{\boldsymbol{\mu}} \times \overrightarrow{\boldsymbol{f}}) \cdot(\overrightarrow{\boldsymbol{\mu}} \times \overrightarrow{\boldsymbol{f}})\right],
$$

while equation (17) becomes:

$$
\begin{gathered}
{\left[C_{n} C_{i}\right] \frac{d C_{i}}{d t}=-\frac{\partial \Delta \mathcal{H}^{(o s c)}}{\partial C_{n}}} \\
+\overrightarrow{\boldsymbol{\mu}} \cdot\left(\frac{\partial \overrightarrow{\boldsymbol{f}}}{\partial C_{n}} \times \overrightarrow{\mathbf{g}}-\overrightarrow{\boldsymbol{f}} \times \frac{\partial \overrightarrow{\mathbf{g}}}{\partial C_{n}}\right)-\dot{\overrightarrow{\boldsymbol{\mu}}} \cdot\left(\overrightarrow{\boldsymbol{f}} \times \frac{\partial \overrightarrow{\boldsymbol{f}}}{\partial C_{n}}\right)-(\overrightarrow{\boldsymbol{\mu}} \times \overrightarrow{\boldsymbol{f}}) \frac{\partial}{\partial C_{n}}(\overrightarrow{\boldsymbol{\mu}} \times \overrightarrow{\boldsymbol{f}}) .
\end{gathered}
$$

To ease the comparison of this equation with (43), it is convenient to split the expression for $\Delta \mathcal{H}^{(\text {osc })}$ in (52) into two parts:

$$
\Delta \mathcal{H}^{(\text {cont })}=-\left[R_{\text {oblate }}(\overrightarrow{\boldsymbol{f}}, t)+\overrightarrow{\boldsymbol{\mu}} \cdot(\overrightarrow{\boldsymbol{f}} \times \overrightarrow{\mathbf{g}})\right]
$$

and

$$
-(\overrightarrow{\boldsymbol{\mu}} \times \overrightarrow{\boldsymbol{f}}) \cdot(\overrightarrow{\boldsymbol{\mu}} \times \overrightarrow{\boldsymbol{f}})
$$

and then to group the latter part with the last term on the right-hand side of (53):

$$
\begin{gathered}
{\left[C_{n} C_{i}\right] \frac{d C_{i}}{d t}=-\frac{\partial \Delta \mathcal{H}^{(c o n t)}}{\partial C_{n}}} \\
+\overrightarrow{\boldsymbol{\mu}} \cdot\left(\frac{\partial \overrightarrow{\boldsymbol{f}}}{\partial C_{n}} \times \overrightarrow{\mathbf{g}}-\overrightarrow{\boldsymbol{f}} \times \frac{\partial \overrightarrow{\mathbf{g}}}{\partial C_{n}}\right)-\dot{\overrightarrow{\boldsymbol{\mu}}} \cdot\left(\overrightarrow{\boldsymbol{f}} \times \frac{\partial \overrightarrow{\boldsymbol{f}}}{\partial C_{n}}\right)+(\overrightarrow{\boldsymbol{\mu}} \times \overrightarrow{\boldsymbol{f}}) \frac{\partial}{\partial C_{n}}(\overrightarrow{\boldsymbol{\mu}} \times \overrightarrow{\boldsymbol{f}}) .
\end{gathered}
$$

One other option is to fully absorb the $O\left(|\overrightarrow{\boldsymbol{\mu}}|^{2}\right)$ term into $\Delta \mathcal{H}$, i.e., to introduce the effective "Hamiltonian"

$$
\Delta \mathcal{H}^{(e f f)}=-\left[R_{\text {oblate }}(\nu)+\overrightarrow{\boldsymbol{\mu}} \cdot(\overrightarrow{\boldsymbol{f}} \times \overrightarrow{\mathbf{g}})+\frac{1}{2}(\overrightarrow{\boldsymbol{\mu}} \times \overrightarrow{\boldsymbol{f}}) \cdot(\overrightarrow{\boldsymbol{\mu}} \times \overrightarrow{\boldsymbol{f}})\right]
$$

and to write down the equations like this:

$$
\left[C_{n} C_{i}\right] \frac{d C_{i}}{d t}=-\frac{\partial \Delta \mathcal{H}^{(e f f)}}{\partial C_{n}}+\overrightarrow{\boldsymbol{\mu}} \cdot\left(\frac{\partial \overrightarrow{\boldsymbol{f}}}{\partial C_{n}} \times \overrightarrow{\mathrm{g}}-\overrightarrow{\boldsymbol{f}} \times \frac{\partial \overrightarrow{\mathbf{g}}}{\partial C_{n}}\right)-\dot{\overrightarrow{\boldsymbol{\mu}}} \cdot\left(\overrightarrow{\boldsymbol{f}} \times \frac{\partial \overrightarrow{\boldsymbol{f}}}{\partial C_{n}}\right)
$$

13 Just as $\Delta \mathcal{H}^{(\text {cont })}$ in (42), this Hamiltonian variation is still equal to $-[R(\overrightarrow{\boldsymbol{f}}, t)+\overrightarrow{\boldsymbol{\mu}} \cdot \overrightarrow{\mathbf{J}}]=$ $-[R(\overrightarrow{\boldsymbol{f}}, t)+\overrightarrow{\boldsymbol{\mu}} \cdot(\overrightarrow{\boldsymbol{f}} \times \overrightarrow{\mathbf{p}})]$. However, the canonical momentum now is different from $\overrightarrow{\mathrm{g}}$ and reads as: $\overrightarrow{\mathrm{p}}=\overrightarrow{\mathrm{g}}+(\overrightarrow{\boldsymbol{\mu}} \times \vec{f})$. 
For $C_{i}$ being chosen as the Keplerian elements, inversion of the Lagrange brackets will yield the following Lagrange-type system:

$$
\frac{d a}{d t}=\frac{2}{n a}\left[\frac{\partial\left(-\Delta \mathcal{H}^{(e f f)}\right)}{\partial M_{o}}-\dot{\overrightarrow{\boldsymbol{\mu}}} \cdot\left(\overrightarrow{\boldsymbol{f}} \times \frac{\partial \overrightarrow{\boldsymbol{f}}}{\partial M_{o}}\right)\right],
$$

$$
\begin{aligned}
& \frac{d e}{d t}=\frac{1-e^{2}}{n a^{2} e}\left[\frac{\partial\left(-\Delta \mathcal{H}^{(e f f)}\right)}{\partial M_{o}}-\dot{\overrightarrow{\boldsymbol{\mu}}} \cdot\left(\overrightarrow{\boldsymbol{f}} \times \frac{\partial \overrightarrow{\boldsymbol{f}}}{\partial M_{o}}\right)\right] \\
& -\frac{\left(1-e^{2}\right)^{1 / 2}}{n a^{2} e}\left[\frac{\partial\left(-\Delta \mathcal{H}^{(e f f)}\right)}{\partial \omega}+\overrightarrow{\boldsymbol{\mu}} \cdot\left(\frac{\partial \overrightarrow{\boldsymbol{f}}}{\partial \omega} \times \overrightarrow{\mathbf{g}}-\overrightarrow{\boldsymbol{f}} \times \frac{\partial \overrightarrow{\mathbf{g}}}{\partial \omega}\right)-\dot{\overrightarrow{\boldsymbol{\mu}}} \cdot\left(\overrightarrow{\boldsymbol{f}} \times \frac{\partial \overrightarrow{\boldsymbol{f}}}{\partial \omega}\right)\right],
\end{aligned}
$$

$$
\frac{d \omega}{d t}=\frac{-\cos i}{n a^{2}\left(1-e^{2}\right)^{1 / 2} \sin i}\left[\frac{\partial\left(-\Delta \mathcal{H}^{(e f f)}\right)}{\partial i}+\overrightarrow{\boldsymbol{\mu}} \cdot\left(\frac{\partial \overrightarrow{\boldsymbol{f}}}{\partial i} \times \overrightarrow{\mathrm{g}}-\overrightarrow{\boldsymbol{f}} \times \frac{\partial \overrightarrow{\mathbf{g}}}{\partial i}\right)-\dot{\overrightarrow{\boldsymbol{\mu}}} \cdot\left(\overrightarrow{\boldsymbol{f}} \times \frac{\partial \overrightarrow{\boldsymbol{f}}}{\partial i}\right)\right]
$$

$$
+\frac{\left(1-e^{2}\right)^{1 / 2}}{n a^{2} e}\left[\frac{\partial\left(-\Delta \mathcal{H}^{(e f f)}\right)}{\partial e}+\overrightarrow{\boldsymbol{\mu}} \cdot\left(\frac{\partial \overrightarrow{\boldsymbol{f}}}{\partial e} \times \overrightarrow{\mathbf{g}}-\overrightarrow{\boldsymbol{f}} \times \frac{\partial \overrightarrow{\mathbf{g}}}{\partial e}\right)-\dot{\overrightarrow{\boldsymbol{\mu}}} \cdot\left(\overrightarrow{\boldsymbol{f}} \times \frac{\partial \overrightarrow{\boldsymbol{f}}}{\partial e}\right)\right],
$$

$$
\begin{aligned}
\frac{d i}{d t}= & \frac{\cos i}{n a^{2}\left(1-e^{2}\right)^{1 / 2} \sin i}\left[\frac{\partial\left(-\Delta \mathcal{H}^{(e f f)}\right)}{\partial \omega}+\overrightarrow{\boldsymbol{\mu}} \cdot\left(\frac{\partial \overrightarrow{\boldsymbol{f}}}{\partial \omega} \times \overrightarrow{\mathrm{g}}-\overrightarrow{\boldsymbol{f}} \times \frac{\partial \overrightarrow{\mathbf{g}}}{\partial \omega}\right)-\dot{\overrightarrow{\boldsymbol{\mu}}} \cdot\left(\overrightarrow{\boldsymbol{f}} \times \frac{\partial \overrightarrow{\boldsymbol{f}}}{\partial \omega}\right)\right]- \\
& \frac{1}{n a^{2}\left(1-e^{2}\right)^{1 / 2} \sin i}\left[\frac{\partial\left(-\Delta \mathcal{H}^{(e f f)}\right)}{\partial \Omega}+\overrightarrow{\boldsymbol{\mu}} \cdot\left(\frac{\partial \overrightarrow{\boldsymbol{f}}}{\partial \Omega} \times \overrightarrow{\mathbf{g}}-\overrightarrow{\boldsymbol{f}} \times \frac{\partial \overrightarrow{\mathbf{g}}}{\partial \Omega}\right)-\dot{\overrightarrow{\boldsymbol{\mu}}} \cdot\left(\overrightarrow{\boldsymbol{f}} \times \frac{\partial \overrightarrow{\boldsymbol{f}}}{\partial \Omega}\right)\right],
\end{aligned}
$$

$$
\frac{d \Omega}{d t}=\frac{1}{n a^{2}\left(1-e^{2}\right)^{1 / 2} \sin i}\left[\frac{\partial\left(-\Delta \mathcal{H}^{(e f f)}\right)}{\partial i}+\overrightarrow{\boldsymbol{\mu}} \cdot\left(\frac{\partial \overrightarrow{\boldsymbol{f}}}{\partial i} \times \overrightarrow{\mathbf{g}}-\overrightarrow{\boldsymbol{f}} \times \frac{\partial \overrightarrow{\mathbf{g}}}{\partial i}\right)-\dot{\overrightarrow{\boldsymbol{\mu}}} \cdot\left(\overrightarrow{\boldsymbol{f}} \times \frac{\partial \overrightarrow{\boldsymbol{f}}}{\partial i}\right)\right]
$$




$$
\begin{aligned}
\frac{d M_{o}}{d t}=- & \frac{1-e^{2}}{n a^{2} e}\left[\frac{\partial\left(-\Delta \mathcal{H}^{(e f f)}\right)}{\partial e}+\overrightarrow{\boldsymbol{\mu}} \cdot\left(\frac{\partial \overrightarrow{\boldsymbol{f}}}{\partial e} \times \overrightarrow{\mathrm{g}}-\overrightarrow{\boldsymbol{f}} \times \frac{\partial \overrightarrow{\mathbf{g}}}{\partial e}\right)-\dot{\overrightarrow{\boldsymbol{\mu}}} \cdot\left(\overrightarrow{\boldsymbol{f}} \times \frac{\partial \overrightarrow{\boldsymbol{f}}}{\partial e}\right)\right] \\
& -\frac{2}{n a}\left[\frac{\partial\left(-\Delta \mathcal{H}^{(e f f)}\right)}{\partial a}+\overrightarrow{\boldsymbol{\mu}} \cdot\left(\frac{\partial \overrightarrow{\boldsymbol{f}}}{\partial a} \times \overrightarrow{\mathbf{g}}-\overrightarrow{\boldsymbol{f}} \times \frac{\partial \overrightarrow{\mathbf{g}}}{\partial a}\right)-\dot{\overrightarrow{\boldsymbol{\mu}}} \cdot\left(\overrightarrow{\boldsymbol{f}} \times \frac{\partial \overrightarrow{\boldsymbol{f}}}{\partial a}\right)\right],
\end{aligned}
$$

terms $\overrightarrow{\boldsymbol{\mu}} \cdot\left(\left(\partial \overrightarrow{\boldsymbol{f}} / \partial M_{o}\right) \times \overrightarrow{\mathbf{g}}-\left(\partial \overrightarrow{\mathbf{g}} / \partial M_{o}\right) \times \overrightarrow{\boldsymbol{f}}\right)$ being omitted in (59- 600), because these terms vanish identically (see the Appendix). In equations (58-644), $\Delta \mathcal{H}^{(e f f)}$ is given by (57). In a rigorous analysis, with the true-anomaly dependence of all terms in (59-64) taken into account, the Hamiltonian will look, according to (26) - 27) and (30):

$$
\begin{gathered}
\Delta \mathcal{H}^{(e f f)}=-\left[R_{\text {oblate }}(\nu)+\overrightarrow{\boldsymbol{\mu}} \cdot(\overrightarrow{\boldsymbol{f}} \times \overrightarrow{\mathbf{g}})+\frac{1}{2}(\overrightarrow{\boldsymbol{\mu}} \times \overrightarrow{\boldsymbol{f}}) \cdot(\overrightarrow{\boldsymbol{\mu}} \times \overrightarrow{\boldsymbol{f}})\right]= \\
-\frac{G m J_{2}}{2} \frac{\rho^{2}}{r^{3}}\left[1-3 \sin ^{2} i \sin ^{2}(\omega+\nu)\right]-\sqrt{G m a\left(1-e^{2}\right)} \overrightarrow{\boldsymbol{w}} \cdot \overrightarrow{\boldsymbol{\mu}}-\frac{1}{2}(\overrightarrow{\boldsymbol{\mu}} \times \overrightarrow{\boldsymbol{f}}) \cdot(\overrightarrow{\boldsymbol{\mu}} \times \overrightarrow{\boldsymbol{f}}) \\
=-\frac{G m J_{2}}{2} \frac{\rho^{2}}{a^{3}}\left(\frac{1+e \cos \nu}{1-e^{2}}\right)^{3}\left[1-3 \sin ^{2} i \sin ^{2}(\omega+\nu)\right] \\
-\sqrt{G m a\left(1-e^{2}\right)}\left(\mu_{1} \sin i \sin \Omega-\mu_{2} \sin i \cos \Omega+\mu_{3} \cos i\right) \\
-\frac{1}{2}(\overrightarrow{\boldsymbol{\mu}} \times \overrightarrow{\boldsymbol{f}}) \cdot(\overrightarrow{\boldsymbol{\mu}} \times \overrightarrow{\boldsymbol{f}})
\end{gathered}
$$

Otherwise, when all terms on the right-hand side of (59 - 64) are substituted with their orbital averages (denoted with the $\langle\ldots\rangle$ symbol), the Hamiltonian will be, due to (31):

$$
\begin{aligned}
& \Delta \mathcal{H}^{(e f f)}=-\left[\left\langle R_{\text {oblate }}\right\rangle+\overrightarrow{\boldsymbol{\mu}} \cdot(\overrightarrow{\boldsymbol{f}} \times \overrightarrow{\mathbf{g}})+\frac{1}{2}\langle(\overrightarrow{\boldsymbol{\mu}} \times \overrightarrow{\boldsymbol{f}}) \cdot(\overrightarrow{\boldsymbol{\mu}} \times \overrightarrow{\boldsymbol{f}})\rangle\right] \\
& =-\frac{G m J_{2}}{4} \frac{\rho^{2}}{a^{3}} \frac{3 \cos ^{2} i-1}{\left(1-e^{2}\right)^{3 / 2}}-\sqrt{G m a\left(1-e^{2}\right)}\left(\mu_{1} \sin i \sin \Omega-\mu_{2} \sin i \cos \Omega+\mu_{3} \cos i\right) \\
& -\frac{1}{2}\langle(\overrightarrow{\boldsymbol{\mu}} \times \overrightarrow{\boldsymbol{f}}) \cdot(\overrightarrow{\boldsymbol{\mu}} \times \overrightarrow{\boldsymbol{f}})\rangle .
\end{aligned}
$$


The expression for $\overrightarrow{\boldsymbol{f}} \times \overrightarrow{\mathbf{g}}$ is true-anomaly-independent and, therefore, does not need to be bracketed with the averaging symbols $\langle\ldots\rangle$. The expression for $(\overrightarrow{\boldsymbol{\mu}} \times \overrightarrow{\boldsymbol{f}}) \cdot(\overrightarrow{\boldsymbol{\mu}} \times \overrightarrow{\boldsymbol{f}})$ through the orbital elements is too cumbersome, and here we do not write it down explicitly. (See formula (186) in the Appendix.) When we permit ourselves to neglect the $O\left(|\overrightarrow{\boldsymbol{\mu}}|^{2}\right)$ inputs, all three Hamiltonians, $\Delta \mathcal{H}^{(o s c)}, \Delta \mathcal{H}^{(\text {cont })}$, and $\Delta \mathcal{H}^{(e f f)}$ coincide:

$$
\begin{gathered}
\Delta \mathcal{H}^{(e f f)} \approx \Delta \mathcal{H}^{(o s c)} \approx \Delta \mathcal{H}^{(\text {cont })}=-\left[\left\langle R_{\text {oblate }}\right\rangle+\overrightarrow{\boldsymbol{\mu}} \cdot(\overrightarrow{\boldsymbol{f}} \times \overrightarrow{\mathbf{g}})\right] \\
=-\frac{G m J_{2}}{4} \frac{\rho^{2}}{a^{3}} \frac{3 \cos ^{2} i-1}{\left(1-e^{2}\right)^{3 / 2}}-\sqrt{G m a\left(1-e^{2}\right)}\left(\mu_{1} \sin i \sin \Omega-\mu_{2} \sin i \cos \Omega+\mu_{3} \cos i\right) .
\end{gathered}
$$

Two important issues should be dwelt upon at this point.

First, we would remind the reader that the function $\Delta \mathcal{H}^{(\text {cont })}$, given by expression (42), yields the correct functional form of the Hamiltonian only in case we express the Hamiltonian through the contact elements (and calculate these through (43) or (46- 51)). In the currently considered case of osculating elements, this $\Delta \mathcal{H}^{(\text {cont })}$ is NOT the correct expression for the Hamiltonian. The correct functional dependence of the Hamiltonian upon the osculating elements, $\Delta \mathcal{H}^{(o s c)}$, is given by formula (52). Though in this dynamical problem the Hamiltonian is unique, its functional dependencies through the contact and through the osculating elements differ from one another, one being $\Delta \mathcal{H}^{(\text {cont) }}$ as in (42), another being $\Delta \mathcal{H}^{(o s c)}$ as in (52). As for the function $\Delta \mathcal{H}^{(e f f)}$ rendered by (57), it is not really a Hamiltonian, but is simply a convenient mathematical entity. In the approximation, where $O\left(|\overrightarrow{\boldsymbol{\mu}}|^{2}\right)$ terms are neglected, there is no difference between these three functions. Despite this, the $O(|\overrightarrow{\boldsymbol{\mu}}|)$ and $O(|\dot{\vec{\mu}}|)$ terms do stay in equations (58 - 64) for osculating elements.

Second, we would comment on our use of expressions (29 - 30) in our derivation of (65) - 67). Above, in subsections 2.2 and 3.1, the use of (27) and (30) was based on formula (29) which interconnected $\overrightarrow{\mathbf{J}}=\overrightarrow{\boldsymbol{r}} \times \overrightarrow{\boldsymbol{p}}=\overrightarrow{\boldsymbol{f}} \times(\dot{\overrightarrow{\boldsymbol{r}}}+\overrightarrow{\boldsymbol{\mu}} \times \overrightarrow{\boldsymbol{f}})$ with contact elements $a, e, i$, and $\Omega$. As demonstrated in Efroimsky \& Goldreich (2004), in that frame $\dot{\overrightarrow{\boldsymbol{r}}}=\overrightarrow{\boldsymbol{g}}-\overrightarrow{\boldsymbol{\mu}} \times \overrightarrow{\boldsymbol{f}}$. Hence, formula (29) interconnects the contact elements with $\overrightarrow{\mathbf{J}}=\overrightarrow{\boldsymbol{f}} \times \overrightarrow{\boldsymbol{g}}$. In the present subsection, we use formula (29) in its usual capacity, i.e., to interconnect $\overrightarrow{\mathbf{J}}=\overrightarrow{\boldsymbol{r}} \times \dot{\overrightarrow{\boldsymbol{r}}}=\overrightarrow{\boldsymbol{f}} \times \overrightarrow{\boldsymbol{g}}$ with osculating elements $a, e, i$, and $\Omega$. It may seem confusing that, though in both cases this formula can be written down in the same way,

$$
\overrightarrow{\boldsymbol{f}} \times \overrightarrow{\boldsymbol{g}}=\sqrt{G m a\left(1-e^{2}\right)} \overrightarrow{\boldsymbol{w}},
$$

its meaning is so different. The clue to understanding this difference lies in the fact that in one case $\dot{\overrightarrow{\boldsymbol{r}}}=\overrightarrow{\boldsymbol{g}}-\overrightarrow{\boldsymbol{\mu}} \times \overrightarrow{\boldsymbol{f}}$ (and, therefore, the elements are contact), while in the other case $\dot{\overrightarrow{\boldsymbol{r}}}=\overrightarrow{\boldsymbol{g}}$ (which makes the elements osculating). For more details see Efroimsky \& Goldreich (2004). 


\section{Comparison of the orbital calculations, performed in terms of the contact elements, with those performed in terms of the osculating elements. The simplest approximation.}

As explained in Section 2, it follows from equations (23 - 24) that the initially small inclination remains so in the course of the oblate primary's precession. Whether this famous result may be interpreted as keeping the satellites in the near-equatorial zone of a precessing planet will depend upon how well the non-osculating (contact) inclination emerging in (23 36) approximates the physical, osculating, inclination rendered by (59- 64).

\subsection{The averaged planetary equations}

Comparing equations (59 - 64) for osculating elements with equations (46 - 51) for contact elements, we immediately see that they differ already in the first order over the precession rate $\overrightarrow{\boldsymbol{\mu}}$ and, therefore, the values of the contact elements will differ from those of their osculating counterparts in the first order, too. A thorough investigation of this difference would demand numerical implementation of both systems and would be extremely timeconsuming. Meanwhile, we can get some preliminary estimates by asking the following, simplified, question: how will the secular, i.e., averaged over an orbital period, components of the contact and orbital elements differ from one another? To answer this question, we perform the following approximations:

1. In equations (59-64), we substitute both the $R_{\text {oblate }}$ term in the Hamiltonian and the $\overrightarrow{\boldsymbol{\mu}}$-dependent terms with their averages (so that, for example, the $R_{\text {oblate }}$ term will be now substituted with $\left\langle R_{\text {oblate }}\right\rangle$ expressed via (31) ).

2. We neglect the terms of order $\overrightarrow{\boldsymbol{\mu}}^{2}$. This way, we restrict the length of time scales involved. (Over sufficiently long times even small terms may accumulate to a noticeable secular correction.) However, we can now benefit from the approximate equality (67).

So truncated and averaged system of Lagrange-type equations will read:

$$
\begin{aligned}
& \frac{d a}{d t}=\frac{2}{n a}\left[-\left\langle\dot{\overrightarrow{\boldsymbol{\mu}}}\left(\overrightarrow{\boldsymbol{f}} \times \frac{\partial \overrightarrow{\boldsymbol{f}}}{\partial M_{o}}\right)\right\rangle\right] \\
& \frac{d e}{d t}=\frac{1-e^{2}}{n a^{2} e}\left[-\left\langle\dot{\overrightarrow{\boldsymbol{\mu}}}\left(\overrightarrow{\boldsymbol{f}} \times \frac{\partial \overrightarrow{\boldsymbol{f}}}{\partial M_{o}}\right)\right\rangle\right] \\
& -\frac{\left(1-e^{2}\right)^{1 / 2}}{n a^{2} e}\left[\left\langle\overrightarrow{\boldsymbol{\mu}} \cdot\left(\frac{\partial \overrightarrow{\boldsymbol{f}}}{\partial \omega} \times \overrightarrow{\mathbf{g}}-\overrightarrow{\boldsymbol{f}} \times \frac{\partial \overrightarrow{\mathbf{g}}}{\partial \omega}\right)\right\rangle-\left\langle\dot{\overrightarrow{\boldsymbol{\mu}}}\left(\overrightarrow{\boldsymbol{f}} \times \frac{\partial \overrightarrow{\boldsymbol{f}}}{\partial \omega}\right)\right\rangle\right],
\end{aligned}
$$




$$
\frac{d \omega}{d t}=\frac{-\cos i}{n a^{2}\left(1-e^{2}\right)^{1 / 2} \sin i}\left[\frac{\partial\left(-\Delta \mathcal{H}^{(e f f)}\right)}{\partial i}+\left\langle\overrightarrow{\boldsymbol{\mu}} \cdot\left(\frac{\partial \overrightarrow{\boldsymbol{f}}}{\partial i} \times \overrightarrow{\mathbf{g}}-\overrightarrow{\boldsymbol{f}} \times \frac{\partial \overrightarrow{\mathbf{g}}}{\partial i}\right)\right\rangle-\left\langle\dot{\overrightarrow{\boldsymbol{\mu}}}\left(\overrightarrow{\boldsymbol{f}} \times \frac{\partial \overrightarrow{\boldsymbol{f}}}{\partial i}\right)\right\rangle\right]
$$

$$
+\frac{\left(1-e^{2}\right)^{1 / 2}}{n a^{2} e}\left[\frac{\partial\left(-\Delta \mathcal{H}^{(e f f)}\right)}{\partial e}+\left\langle\overrightarrow{\boldsymbol{\mu}} \cdot\left(\frac{\partial \overrightarrow{\boldsymbol{f}}}{\partial e} \times \overrightarrow{\mathbf{g}}-\overrightarrow{\boldsymbol{f}} \times \frac{\partial \overrightarrow{\mathbf{g}}}{\partial e}\right)\right\rangle-\left\langle\dot{\overrightarrow{\boldsymbol{\mu}}}\left(\overrightarrow{\boldsymbol{f}} \times \frac{\partial \overrightarrow{\boldsymbol{f}}}{\partial e}\right)\right\rangle\right]
$$

$$
\begin{aligned}
& \frac{d i}{d t}=\frac{\cos i}{n a^{2}\left(1-e^{2}\right)^{1 / 2} \sin i}\left[\left\langle\overrightarrow{\boldsymbol{\mu}} \cdot\left(\frac{\partial \overrightarrow{\boldsymbol{f}}}{\partial \omega} \times \overrightarrow{\mathbf{g}}-\overrightarrow{\boldsymbol{f}} \times \frac{\partial \overrightarrow{\mathbf{g}}}{\partial \omega}\right)\right\rangle-\left\langle\dot{\overrightarrow{\boldsymbol{\mu}}}\left(\overrightarrow{\boldsymbol{f}} \times \frac{\partial \overrightarrow{\boldsymbol{f}}}{\partial \omega}\right)\right\rangle-\right. \\
& \frac{1}{n a^{2}\left(1-e^{2}\right)^{1 / 2} \sin i}\left[\frac{\partial\left(-\Delta \mathcal{H}^{(e f f)}\right)}{\partial \Omega}+\left\langle\overrightarrow{\boldsymbol{\mu}} \cdot\left(\frac{\partial \overrightarrow{\boldsymbol{f}}}{\partial \Omega} \times \overrightarrow{\mathbf{g}}-\overrightarrow{\boldsymbol{f}} \times \frac{\partial \overrightarrow{\mathbf{g}}}{\partial \Omega}\right)\right\rangle-\left\langle\dot{\overrightarrow{\boldsymbol{\mu}}}\left(\overrightarrow{\boldsymbol{f}} \times \frac{\partial \overrightarrow{\boldsymbol{f}}}{\partial \Omega}\right)\right\rangle\right],
\end{aligned}
$$

$$
\frac{d \Omega}{d t}=\frac{1}{n a^{2}\left(1-e^{2}\right)^{1 / 2} \sin i}\left[\frac{\partial\left(-\Delta \mathcal{H}^{(e f f)}\right)}{\partial i}+\left\langle\overrightarrow{\boldsymbol{\mu}} \cdot\left(\frac{\partial \overrightarrow{\boldsymbol{f}}}{\partial i} \times \overrightarrow{\mathbf{g}}-\overrightarrow{\boldsymbol{f}} \times \frac{\partial \overrightarrow{\mathbf{g}}}{\partial i}\right)\right\rangle-\left\langle\dot{\overrightarrow{\boldsymbol{\mu}}}\left(\overrightarrow{\boldsymbol{f}} \times \frac{\partial \overrightarrow{\boldsymbol{f}}}{\partial i}\right)\right\rangle\right]
$$

$$
\begin{aligned}
\frac{d M_{o}}{d t}= & -\frac{1-e^{2}}{n a^{2} e}\left[\frac{\partial\left(-\Delta \mathcal{H}^{(e f f)}\right)}{\partial e}+\left\langle\overrightarrow{\boldsymbol{\mu}} \cdot\left(\frac{\partial \overrightarrow{\boldsymbol{f}}}{\partial e} \times \overrightarrow{\mathbf{g}}-\overrightarrow{\boldsymbol{f}} \times \frac{\partial \overrightarrow{\mathbf{g}}}{\partial e}\right)\right\rangle-\left\langle\dot{\overrightarrow{\boldsymbol{\mu}}}\left(\overrightarrow{\boldsymbol{f}} \times \frac{\partial \overrightarrow{\boldsymbol{f}}}{\partial e}\right)\right\rangle\right] \\
& -\frac{2}{n a}\left[\frac{\partial\left(-\Delta \mathcal{H}^{(e f f)}\right)}{\partial a}+\left\langle\overrightarrow{\boldsymbol{\mu}} \cdot\left(\frac{\partial \overrightarrow{\boldsymbol{f}}}{\partial a} \times \overrightarrow{\mathbf{g}}-\overrightarrow{\boldsymbol{f}} \times \frac{\partial \overrightarrow{\mathbf{g}}}{\partial a}\right)\right\rangle-\left\langle\dot{\overrightarrow{\boldsymbol{\mu}}}\left(\overrightarrow{\boldsymbol{f}} \times \frac{\partial \overrightarrow{\boldsymbol{f}}}{\partial a}\right)\right\rangle\right],
\end{aligned}
$$

the Hamiltonian here being approximated by (67), and the angular brackets signifying orbital averaging. In equations (69), (70) and (72) we took into account that the averaged 
Hamiltonian (67) bears no dependence upon $M_{o}$ and $\omega$. (This, though, will not be the case for the exact, $\nu$-dependent, Hamiltonian given by (52) and (26) ! )

Calculation of $\left(\left(\partial \overrightarrow{\boldsymbol{f}} / \partial C_{j}\right) \times \overrightarrow{\mathbf{g}}-\left(\partial \overrightarrow{\mathbf{g}} / \partial C_{j}\right) \times \overrightarrow{\boldsymbol{f}}\right)$ and $-\dot{\overrightarrow{\boldsymbol{\mu}}}\left(\overrightarrow{\boldsymbol{f}} \times \partial \overrightarrow{\mathbf{g}} / \partial C_{j}\right)$ takes pages of algebra. A short synopsis of this calculation is offered in the Appendix. Here follows the outcome:

$$
\begin{aligned}
& \overrightarrow{\boldsymbol{\mu}} \cdot\left(\frac{\partial \overrightarrow{\boldsymbol{f}}}{\partial a} \times \overrightarrow{\mathbf{g}}-\overrightarrow{\boldsymbol{f}} \times \frac{\partial \overrightarrow{\mathbf{g}}}{\partial a}\right)=\frac{3}{2} \mu_{\perp} \sqrt{\frac{G m\left(1-e^{2}\right)}{a}} \\
& \overrightarrow{\boldsymbol{\mu}} \cdot\left(\frac{\partial \overrightarrow{\boldsymbol{f}}}{\partial e} \times \overrightarrow{\mathbf{g}}-\overrightarrow{\boldsymbol{f}} \times \frac{\partial \overrightarrow{\mathbf{g}}}{\partial e}\right)=-\mu_{\perp} \frac{n a^{2}\left(3 e+2 \cos \nu+e^{2} \cos \nu\right)}{(1+e \cos \nu) \sqrt{1-e^{2}}} \\
& \overrightarrow{\boldsymbol{\mu}} \cdot\left(\frac{\partial \overrightarrow{\boldsymbol{f}}}{\partial \omega} \times \overrightarrow{\mathrm{g}}-\overrightarrow{\boldsymbol{f}} \times \frac{\partial \overrightarrow{\mathbf{g}}}{\partial \omega}\right)=-2 \mu_{\perp} \frac{n a^{2} \sqrt{1-e^{2}}}{1+e \cos \nu} e \sin \nu \\
& \overrightarrow{\boldsymbol{\mu}} \cdot\left(\frac{\partial \overrightarrow{\boldsymbol{f}}}{\partial \Omega} \times \overrightarrow{\mathrm{g}}-\overrightarrow{\boldsymbol{f}} \times \frac{\partial \overrightarrow{\mathbf{g}}}{\partial \Omega}\right)= \\
& \mu_{1} \quad\left[\frac{n a^{2} \sqrt{1-e^{2}}}{1+e \cos \nu} \sin i \quad\{\cos \Omega \cos [2(\omega+\nu)]-\sin \Omega \cos i \sin [2(\omega+\nu)]\}+\right. \\
& \left.\frac{n a^{2} \sqrt{1-e^{2}}}{1+e \cos \nu} e \sin i \quad\{\cos \Omega \cos (\nu+2 \omega)-2 \sin \Omega \cos i \sin (\omega+\nu) \cos \omega\}\right]+ \\
& \mu_{2}\left[\frac{n a^{2} \sqrt{1-e^{2}}}{1+e \cos \nu} \sin i \quad\{\sin \Omega \cos [2(\omega+\nu)]+\cos \Omega \cos i \sin [2(\omega+\nu)]\}+\right. \\
& \left.\frac{n a^{2} \sqrt{1-e^{2}}}{1+e \cos \nu} e \sin i\{\sin \Omega \cos (\nu+2 \omega)+2 \cos \Omega \cos i \sin (\omega+\nu) \cos \omega\}\right]+ \\
& \mu_{3}\left[\frac{n a^{2} \sqrt{1-e^{2}}}{1+e \cos \nu} \sin ^{2} i \sin [2(\omega+\nu)]+\frac{n a^{2} \sqrt{1-e^{2}}}{1+e \cos \nu} 2 e\left\{-\sin \nu+\sin ^{2} i \cos \omega \sin (\omega+\nu)\right\}\right]
\end{aligned}
$$


$\overrightarrow{\boldsymbol{\mu}} \cdot\left(\frac{\partial \overrightarrow{\boldsymbol{f}}}{\partial i} \times \overrightarrow{\mathrm{g}}-\overrightarrow{\boldsymbol{f}} \times \frac{\partial \overrightarrow{\mathbf{g}}}{\partial i}\right)=$

$\mu_{1} \frac{n a^{2} \sqrt{1-e^{2}}}{1+e \cos \nu}\left\{\left[(-\sin \Omega \cos i \cos 2 \omega-\cos \Omega \sin 2 \omega)\left(\cos ^{2} \nu-\sin ^{2} \nu\right)+\right.\right.$

$2 \sin \nu \cos \nu(\sin \Omega \cos i \sin 2 \omega-\cos \Omega \cos 2 \omega)]+$

$\left.e\left[(-\sin \Omega \cos i \cos 2 \omega-\cos \Omega \sin 2 \omega) \cos \nu+\left(\sin \Omega \cos i \sin 2 \omega-2 \cos \Omega \cos ^{2} \omega\right) \sin \nu\right]\right\}$

$+\mu_{2} \frac{n a^{2} \sqrt{1-e^{2}}}{1+e \cos \nu}\left\{\left[(\cos \Omega \cos i \cos 2 \omega-\sin \Omega \sin 2 \omega)\left(\cos ^{2} \nu-\sin ^{2} \nu\right)+\right.\right.$

$2 \sin \nu \cos \nu(-\cos \Omega \cos i \sin 2 \omega-\sin \Omega \cos 2 \omega)]+$

$\left.e\left[(\cos \Omega \cos i \cos 2 \omega-\sin \Omega \sin 2 \omega) \cos \nu+\left(-2 \sin \Omega \cos ^{2} \omega+\cos \Omega \sin 2 \omega \cos i\right) \sin \nu\right]\right\}$

$+\mu_{3} \frac{n a^{2} \sqrt{1-e^{2}}}{1+e \cos \nu}\left\{\sin i\left[\cos 2 \omega\left(\cos ^{2} \nu-\sin ^{2} \nu\right)-2 \sin 2 \omega \sin \nu \cos \nu\right]+\right.$

$e[\sin i \cos 2 \omega \cos \nu-\sin i \sin 2 \omega \sin \nu]\}$,

$$
\begin{aligned}
& \overrightarrow{\boldsymbol{\mu}} \cdot\left(\frac{\partial \overrightarrow{\boldsymbol{f}}}{\partial M_{o}} \times \overrightarrow{\mathrm{g}}-\overrightarrow{\boldsymbol{f}} \times \frac{\partial \overrightarrow{\mathbf{g}}}{\partial M_{o}}\right)=0, \\
& -\dot{\overrightarrow{\boldsymbol{\mu}}} \cdot\left(\overrightarrow{\boldsymbol{f}} \times \frac{\partial \overrightarrow{\boldsymbol{f}}}{\partial a}\right)=\frac{3}{2} \dot{\mu}_{\perp} a n\left(t-t_{o}\right) \sqrt{1-e^{2}},
\end{aligned}
$$




$$
\begin{aligned}
& -\dot{\overrightarrow{\boldsymbol{\mu}}} \cdot\left(\overrightarrow{\boldsymbol{f}} \times \frac{\partial \overrightarrow{\boldsymbol{f}}}{\partial e}\right)=-\dot{\mu}_{\perp} a^{2} \frac{\left(1-e^{2}\right)}{1+e \cos \nu} \sin \nu \\
& -\dot{\overrightarrow{\boldsymbol{\mu}}} \cdot\left(\overrightarrow{\boldsymbol{f}} \times \frac{\partial \overrightarrow{\boldsymbol{f}}}{\partial \omega}\right)=-\dot{\mu}_{\perp} a^{2} \frac{\left(1-e^{2}\right)^{2}}{(1+e \cos \nu)^{2}} \\
& -\overrightarrow{\boldsymbol{\mu}} \cdot\left(\overrightarrow{\boldsymbol{f}} \times \frac{\partial \overrightarrow{\boldsymbol{f}}}{\partial \Omega}\right)= \\
& a^{2} \frac{\left(1-e^{2}\right)^{2}}{(1+e \cos \nu)^{2}}\left\{\dot{\mu}_{1}[\cos \Omega \cos (\omega+\nu)-\sin \Omega \sin (\omega+\nu) \cos i] \sin (\omega+\nu) \sin i\right. \\
& +\dot{\mu}_{2}[\sin \Omega \cos (\omega+\nu)+\cos \Omega \sin (\omega+\nu) \cos i] \sin (\omega+\nu) \sin i \\
& \left.+\dot{\mu}_{3}\left[\cos ^{2}(\omega+\nu)+\sin ^{2}(\omega+\nu) \cos ^{2} i\right]\right\} \\
& -\overrightarrow{\boldsymbol{\mu}} \cdot\left(\overrightarrow{\boldsymbol{f}} \times \frac{\partial \overrightarrow{\boldsymbol{f}}}{\partial i}\right)= \\
& a^{2} \frac{\left(1-e^{2}\right)^{2}}{(1+e \cos \nu)^{2}}\left\{\dot{\mu}_{1}[-\cos \Omega \sin (\omega+\nu)-\sin \Omega \cos (\omega+\nu) \cos i] \sin (\omega+\nu)\right. \\
& +\dot{\mu}_{2}[-\sin \Omega \sin (\omega+\nu)+\cos \Omega \cos (\omega+\nu) \cos i] \sin (\omega+\nu) \\
& \left.+\dot{\mu}_{3} \sin (\omega+\nu) \cos (\omega+\nu) \sin i\right\} \\
& -\overrightarrow{\boldsymbol{\mu}} \cdot\left(\overrightarrow{\boldsymbol{f}} \times \frac{\partial \overrightarrow{\boldsymbol{f}}}{\partial M_{o}}\right)=-\dot{\mu}_{\perp} a^{2} \sqrt{1-e^{2}}
\end{aligned}
$$


$\mu_{1}, \mu_{2}$, and $\mu_{3}$ being the Cartesian components of the precession rate, as seen in the coprecessing frame, and $\mu_{\perp}$ being the component of the precession rate, aimed in the direction of the angular momentum; it is given by (117).

It may seem strange that the right-hand side of (76) does not vanish in the limit of $e \rightarrow 0$. The absurdity of this will be easily redeemed by the fact that this term shows up only in the equation for $d \omega / d t$ and, therefore, leads to no physical paradoxes in the limit of a circular orbit. However, for finite values of the eccentricity, this term contributes to the periapse precession.

Another seemingly calamitous thing is the divergence in (82). This divergence, however, entails no disastrous physical consequences, because the term (82) shows up only in the planetary equation for $d M_{o} / d t$ and simply leads to a steady shift of the initial condition $M_{o}$.

\subsection{The case of a constant precession rate.}

The situation might simplify very considerably if we could also assume that the precession rate $\overrightarrow{\boldsymbol{\mu}}$ stays constant. Then in equations (69-740), we would take $\overrightarrow{\boldsymbol{\mu}}$ out of the angular brackets and proceed with averaging the expressions $\left(\left(\partial \overrightarrow{\boldsymbol{f}} / \partial C_{j}\right) \times \overrightarrow{\mathbf{g}}-\overrightarrow{\boldsymbol{f}} \times\left(\partial \overrightarrow{\mathbf{g}} / \partial C_{j}\right)\right)$ only (while all the terms with $\dot{\overrightarrow{\boldsymbol{\mu}}}$ will now vanish). It is, of course, well known that this is physically wrong, because the planetary precession has a continuous spectrum of frequencies (some of which are commensurate with the orbital frequency of the satellite) 14 Nevertheless, for the sake of argument let us go on with this assumption.

The averaging of (75) and (80) is self-evident. The averaging of (76- 79) is lengthy and is presented in the Appendix. All in all, we get, for constant $\overrightarrow{\boldsymbol{\mu}}$ :

$$
\begin{aligned}
& \overrightarrow{\boldsymbol{\mu}} \cdot\left\langle\left(\frac{\partial \overrightarrow{\boldsymbol{f}}}{\partial a} \times \overrightarrow{\mathbf{g}}-\overrightarrow{\boldsymbol{f}} \times \frac{\partial \overrightarrow{\mathbf{g}}}{\partial a}\right)\right\rangle=\overrightarrow{\boldsymbol{\mu}} \cdot\left(\frac{\partial \overrightarrow{\boldsymbol{f}}}{\partial a} \times \overrightarrow{\mathbf{g}}-\overrightarrow{\boldsymbol{f}} \times \frac{\partial \overrightarrow{\mathbf{g}}}{\partial a}\right)=\frac{3}{2} \mu_{\perp} \sqrt{\frac{G m\left(1-e^{2}\right)}{a}} \\
& \overrightarrow{\boldsymbol{\mu}} \cdot\left\langle\left(\frac{\partial \overrightarrow{\boldsymbol{f}}}{\partial C_{j}} \times \overrightarrow{\mathbf{g}}-\overrightarrow{\boldsymbol{f}} \times \frac{\partial \overrightarrow{\mathbf{g}}}{\partial C_{j}}\right)\right\rangle=0 \quad, \quad C_{j}=e, \Omega, \omega, i, M_{o}
\end{aligned}
$$

Since the orbital averages (88) vanish, then $e$ will, along with $a$, stay constant for as long as our approximation remains valid. Besides, no trace of $\overrightarrow{\boldsymbol{\mu}}$ will be left in the equations for $\Omega$ and $i$. This means that, in the assumed approximation and under the extra assumption of constant $\overrightarrow{\boldsymbol{\mu}}$, the afore-quoted analysis (23] - 36), offered by Goldreich (1965), will remain valid at time scales which are not too long. At longer scales (of order dozens of millions of years and longer) one has to take into consideration the back reaction of the short-period terms upon the secular ones (Laskar 1990). Beside the latter issue, the problem with this approximation is that it ignores both the long-term evolution of the spin axis and the shortterm nutations. For these reasons, this approximation will not be extendable to long periods

\footnotetext{
14 The case of the Earth rotation and precession is comprehensively reviewed by Eubanks (1993). The Martian short-time-scale rotational dynamics is of an equal complexity, even though Mars lacks oceans and the coupling of its rotation with the atmospheric motions is weaker than in the case of the Earth (Defraigne et al 2003, Van Hoolst et al 2000, Dehant et al 2000).
} 
of Mars' evolution. This puts forward the bigger question: what maximal amplitude of obliquity variations could Mars afford, to keep both its satellites so close to its equatorial plane?

Even in the unphysical case of constant $\overrightarrow{\boldsymbol{\mu}}$ the averaged equation (74) for the osculating $M_{o}$ differs already in the first order over $\overrightarrow{\boldsymbol{\mu}}$ from equation (48) for the contact $M_{o}$. In the realistic case of time-dependent precession, the averages of terms containing $\overrightarrow{\boldsymbol{\mu}}$ do not vanish (except for $\overrightarrow{\boldsymbol{\mu}} \cdot\left(\left(\partial \overrightarrow{\boldsymbol{f}} / \partial M_{o}\right) \times \overrightarrow{\mathbf{g}}-\overrightarrow{\boldsymbol{f}} \times\left(\partial \overrightarrow{\mathbf{g}} / \partial M_{o}\right)\right)$ which is identically nil). These terms show up in all equations (except in that for $a$ ) and influence the motion. They will be the key to our understanding the long-term satellite dynamics, including the secular drift of the orbit plane, caused by the precession $\overrightarrow{\boldsymbol{\mu}}$.

\section{An outline of a more accurate analysis. Resonances between the planetary nutation and the satellite or- bital frequency.}

Precession of any planet contains in itself a continuous spectrum of circular frequencies involved:15

$$
\overrightarrow{\boldsymbol{\mu}}(t)=\int_{-\infty}^{\infty} \overrightarrow{\boldsymbol{\mu}}(s) e^{-i s t} d s
$$

some modes being more prominent than the others. For our present purposes, it will be advantageous to express the precession rate as function of the satellite's true anomaly:

$$
\overrightarrow{\boldsymbol{\mu}}(\nu)=\int_{-\infty}^{\infty} \overrightarrow{\boldsymbol{\mu}}(W) e^{-i W \nu} d W
$$

$W$ being the circular "frequency" related to the true anomaly $\nu$. Needless to say, $\overrightarrow{\boldsymbol{\mu}}(t) \overrightarrow{\boldsymbol{\mu}}(\nu), \overrightarrow{\boldsymbol{\mu}}(s)$, and $\overrightarrow{\boldsymbol{\mu}}(W)$ are four different functions. However, we take the liberty of using the same notation $\overrightarrow{\boldsymbol{\mu}}(\ldots)$ because the argument will always reveal which

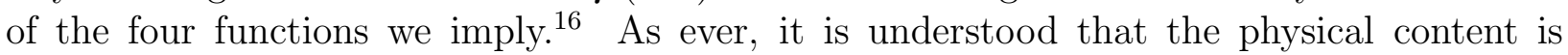
attributed to the real parts of $\overrightarrow{\boldsymbol{\mu}}(t)$ and $\overrightarrow{\boldsymbol{\mu}}(\nu)$.

If we now plug the real part of (90) into (75)- 87) and carry out averaging in accordance with formula (109) of the Appendix, we shall see that the secular parts of these $\overrightarrow{\boldsymbol{\mu}}$-dependent terms do not vanish. They reveal the influence of the planetary precession upon the satellite orbital motion. Especially interesting are the resonant contributions provided by the integer $W$ 's, because these nutation modes are commensurate with the orbital motion of the satellite.

For plugging $\overrightarrow{\boldsymbol{\mu}}(\nu)$ into the terms (75- 86), it will be convenient to rewrite (90) as

$$
\overrightarrow{\boldsymbol{\mu}}(\nu)=\int_{0}^{\infty}\left[\overrightarrow{\boldsymbol{\mu}}^{(s)}(W) \sin (W \nu)+\overrightarrow{\boldsymbol{\mu}}^{(c)}(W) \cos (W \nu)\right] d W .
$$

\footnotetext{
${ }^{15}$ A more honest analysis should take into account also the direct dependence of the planet's precession rate upon the instantaneous position(s) of its satellite(s): $\overrightarrow{\boldsymbol{\mu}}=\overrightarrow{\boldsymbol{\mu}}\left(t ; a, e, \Omega, \omega, i, M_{o}\right)$. However, the back-reaction of the satellites upon the primary is known to be an effect of a higher order of smallness (Laskar 2004), at least in the case of Mars; and therefore we shall omit this circumstance by simply assuming that $\overrightarrow{\boldsymbol{\mu}}=\overrightarrow{\boldsymbol{\mu}}(t)=\overrightarrow{\boldsymbol{\mu}}(t(\nu))$.

${ }^{16}$ Evidently, $\overrightarrow{\boldsymbol{\mu}}(\nu)$ is a short notation for $\overrightarrow{\boldsymbol{\mu}}(t(\nu))$. It is also possible to demonstrate that, in the limit of vanishing eccentricity, $\overrightarrow{\boldsymbol{\mu}}(W) \approx n \overrightarrow{\boldsymbol{\mu}}(W n)$ and $W \approx s / n$.
} 
Insertion of the latter into (75- 86) will still result in extremely sophisticated integrals. For example, the term (75) will have the following orbital average:

$$
\begin{array}{r}
\left\langle\overrightarrow{\boldsymbol{\mu}} \cdot\left(\frac{\partial \overrightarrow{\boldsymbol{f}}}{\partial a} \times \overrightarrow{\mathbf{g}}-\overrightarrow{\boldsymbol{f}} \times \frac{\partial \overrightarrow{\mathbf{g}}}{\partial a}\right)\right\rangle=\frac{3}{2} \sqrt{\frac{G m\left(1-e^{2}\right)}{a}}\left\langle\mu_{\perp}\right\rangle= \\
\frac{3}{4 \pi} \sqrt{\frac{G m}{a}}\left(1-e^{2}\right)^{2} \int_{0}^{\infty} d W \int_{0}^{2 \pi} d \nu \frac{\mu_{\perp}^{(s)}(W) \sin (W \nu)+\mu_{\perp}^{(c)}(W) \cos (W \nu)}{(1+e \cos \nu)^{2}}
\end{array}
$$

(the averaging rule (109) being employed). Evaluation of the two integrals emerging in this expression can, in principle, be carried out in term of the hypergeometric functions, but the outcome will be hard to work with and hard to interpret physically. Even worse integrals will show up in the averages of (76- 86).

To get an idea as to how much the terms (75- 86) contribute to the secular drift of the satellite orbits from the planet's equator, it may be good to first calculate these term's averages under the assumption of small eccentricity. Then, for example, (92) will simplify to

$$
\left\langle\overrightarrow{\boldsymbol{\mu}} \cdot\left(\frac{\partial \overrightarrow{\boldsymbol{f}}}{\partial a} \times \overrightarrow{\mathrm{g}}-\overrightarrow{\boldsymbol{f}} \times \frac{\partial \overrightarrow{\mathbf{g}}}{\partial a}\right)\right\rangle \approx
$$

$$
\begin{aligned}
\frac{3}{4 \pi} \sqrt{\frac{G m}{a}}\left(1-e^{2}\right)^{2} \int_{0}^{\infty} d W \int_{0}^{2 \pi} d \nu & \left\{\mu_{\perp}^{(s)}(W) \sin (W \nu)\left(1-2 e \cos \nu+3 e^{2} \cos ^{2} \nu+\ldots\right)\right. \\
& \left.+\mu_{\perp}^{(c)}(W) \cos (W \nu)\left(1-2 e \cos \nu+3 e^{2} \cos ^{2} \nu+\ldots\right)\right\}
\end{aligned}
$$

We immediately see that the above expression consists of two parts: the non-resonant one and the resonant one.

This topic will be addressed in our subsequent paper, where we shall try to determine how much time is needed for these subtle effects to accumulate enough to cause a substantial secular drift of the orbit plane.

\section{Conclusions}

In this article we have prepared an analytical launching pad for the research of long-term evolution of orbits about a precessing oblate primary. This paper is the first in a series and is technical, so we deliberately avoided making whatever quantitative estimates, leaving those for the next part of our project.

The pivotal question emerging in the context of this research is whether the orbital planes of near-equatorial satellites will drift away from the planetary equator in the cause of the planet's obliquity changes. Several facts have been established in this regard.

First, the planetary equations for osculating elements of the satellite do contain terms responsible for such a drift. These terms contain inputs of the first order and of the second order in $\overrightarrow{\boldsymbol{\mu}}$, and of the first order in $\dot{\overrightarrow{\boldsymbol{\mu}}}$, where $\overrightarrow{\boldsymbol{\mu}}$ is the precession rate of the primary. 
Second, the first-order (but not the second-order) terms average out in the case of a constant precession rate, which means that in this case their effect will accumulate only over extremely long time scales. We would remind that the sort-period terms of the planetary equations do exert back-reaction upon the secular ones. While in the artificial-satellite science, which deals with short interval of time, the short-period terms often may be omitted, in long-term astronomical computations (dozens of million years and higher), the accumulated influence of short-period terms must be taken into account. A simple explanation of how this should be done is offered in section 2 of Laskar (1990). Besides, the contribution of the secular second-order terms will, too, accumulate over very large time intervals.

Third, the first-order drift terms do not average out in the case of variable precession. Under these circumstances they become secular. This means, for example, that the turbulent history of Mars' obliquity - history which includes both long-term changes (Ward 1973, 1974, 1979; Laskar \& Robutel 1993; Touma \& Wisdom 1994) and short-term nutations (Dehant et al 2000; Van Hoolst et al 2000; Defraigne et al 2004) - might have lead to a secular drift of the initially near-equatorial satellites. If that were the case, then the current, still near-equatorial, location of Phobos and Deimos may lead to restrictions upon the rate and amplitude of the Martian obliquity variations. To render a judgment on this topic, one should compute how quickly this drift accumulates. Very likely, this quest will demand heavy-duty numerics.

\section{$\underline{\text { Acknowledgments }}$}

I owe my sincere gratitude to Yurii Batrakov, Peter Goldreich, Pini Gurfil, George Kaplan, Konstantin Kholshevnikov, Marc Murison, Alessandro Morbidelli, William Newman, Victor Slabinski, and Jack Wisdom, all of whom kindly discussed this material with me (and are in no way to blame for my errors of omission or commission, for which I alone am responsible). My great thanks are due to my Department Head, John Bangert, who tolerated the project since its earliest stage, when its success was yet far from evident. This research was supported by NASA grant W-19948. 


\section{Appendix.}

\section{The inertial terms emerging in the planetary equations}

In this Appendix we write down, as functions of the true anomaly, the $\overrightarrow{\boldsymbol{\mu}}$ - and $\dot{\overrightarrow{\boldsymbol{\mu}}}$ dependent terms in (59-64). We also calculate their orbital averages in the case of a constant precession rate $\overrightarrow{\boldsymbol{\mu}}$.

\section{A.1. The basic formulae}

Formulae (55 - 60) contain the two-body unperturbed expressions for the position and velocity as functions of time and the six Keplerian elements, (1) and (7). To find their explicit form, one can employ an auxiliary set of dextral perifocal coordinates $\overrightarrow{\mathbf{q}}$, with an origin at the gravitating centre, and with the first two axes located in the plane of the orbit:

$$
\overrightarrow{\mathbf{q}}=\{r \cos \nu, \quad r \sin \nu, \quad 0\}^{\mathbf{T}}=a \frac{1-e^{2}}{1+e \cos \nu}\{\cos \nu, \quad \sin \nu, \quad 0\}^{\mathbf{T}} .
$$

The corresponding velocities will read:

$$
\begin{gathered}
\dot{\overrightarrow{\mathbf{q}}}=\left\{-\frac{n a \sin \nu}{\sqrt{1-e^{2}}}, \frac{n a(e+\cos \nu)}{\sqrt{1-e^{2}}}, 0\right\}^{\mathbf{T}}= \\
\frac{n a}{\sqrt{1-e^{2}}}\{-\sin \nu, \quad(e+\cos \nu), \quad 0\}^{\mathbf{T}} .
\end{gathered}
$$

the radius in (94) being a function of the semiaxis major $a$, the eccentricity $e$, and the true anomaly $\nu$ :

$$
r=a \frac{1-e^{2}}{1+e \cos \nu}
$$

the true anomaly $\nu$ itself being a function of $a, e$, and of the mean anomaly $M \equiv$ $M_{o}+\int_{t_{o}}^{t} n d t$, where $n \equiv(G m)^{1 / 2} a^{-3 / 2}$. Then, in the two-body setting, the position and velocity, related to some fiducial inertial frame, will appear as:

$$
\begin{aligned}
& \overrightarrow{\boldsymbol{r}}=\overrightarrow{\boldsymbol{f}}\left(C_{1}, \ldots, C_{6}, t\right)=\hat{\mathbf{R}}(\Omega, i, \omega) \overrightarrow{\mathbf{q}}\left(a, e, M_{o}, t\right) \\
& \dot{\overrightarrow{\boldsymbol{r}}}=\overrightarrow{\mathbf{g}}\left(C_{1}, \ldots, C_{6}, t\right)=\hat{\mathbf{R}}(\Omega, i, \omega) \dot{\overrightarrow{\mathbf{q}}}\left(a, e, M_{o}, t\right),
\end{aligned}
$$

$\hat{\mathbf{R}}(\Omega, i, \omega)$ being the matrix of rotation from the orbital-plane-related axes $q$ to some fixed Cartesian axes $\left(x_{1}, x_{2}, x_{3}\right)$ in the said fiducial inertial frame wherein the vectors $\overrightarrow{\mathbf{r}}$ and $\dot{\overrightarrow{\boldsymbol{r}}}$ are defined. The rotation is parameterised by the three Euler angles: inclination, $i$; the longitude of the node, $\Omega$; and the argument of the pericentre, $\omega$. Thence, as is well known (see, for example, Morbidelli (2002), subsection 1.2), 


$$
\begin{aligned}
& \cos \Omega \cos \omega-\sin \Omega \sin \omega \cos i \quad-\cos \Omega \sin \omega-\sin \Omega \cos \omega \cos i \quad \sin \Omega \sin i \\
& \hat{\mathbf{R}}=\quad \sin \Omega \cos \omega+\cos \Omega \sin \omega \cos i \quad-\sin \Omega \sin \omega+\cos \Omega \cos \omega \cos i \quad-\cos \Omega \sin i \\
& \sin \omega \sin i \quad \cos \omega \sin i \quad \cos i
\end{aligned}
$$

insertion whereof, together with (94), into the first equation of (97) yields

$$
\begin{aligned}
& f_{1}=a \frac{1-e^{2}}{1+e \cos \nu}[\cos \Omega \cos (\omega+\nu)-\sin \Omega \sin (\omega+\nu) \cos i] \\
& f_{2}=a \frac{1-e^{2}}{1+e \cos \nu}[\sin \Omega \cos (\omega+\nu)+\cos \Omega \sin (\omega+\nu) \cos i] \\
& f_{3}=a \frac{1-e^{2}}{1+e \cos \nu} \sin (\omega+\nu) \sin i
\end{aligned}
$$

Similarly, substitution of (95) and (98) into the second equation of (97) entails:

$$
\begin{gathered}
g_{1}=\frac{n a}{\sqrt{1-e^{2}}}[-\cos \Omega \sin (\omega+\nu)-\sin \Omega \cos (\omega+\nu) \cos i+ \\
e(-\cos \Omega \sin \omega-\sin \Omega \cos \omega \cos i)] \\
g_{2}=\frac{n a}{\sqrt{1-e^{2}}}[-\sin \Omega \sin (\omega+\nu)+\cos \Omega \cos (\omega+\nu) \cos i+ \\
e(-\sin \Omega \sin \omega+\cos \Omega \cos \omega \cos i)] \\
g_{3}=\frac{n a}{\sqrt{1-e^{2}}} \sin i[\cos (\omega+\nu)+e \cos \omega]
\end{gathered}
$$

the subscripts $1,2,3$ denoting the $x_{1}, x_{2}, x_{3}$ components in the fiducial inertial frame wherein (97) is written.

\section{A.2. The averaging rule}

From equations

$$
\cos E=\frac{e+\cos \nu}{1+e \cos \nu} \quad, \quad \sin E=\frac{\sqrt{1-e^{2}} \sin \nu}{1+e \cos \nu}
$$

it follows that

$$
\frac{\partial E}{\partial \nu}=\frac{\sqrt{1-e^{2}}}{1+e \cos \nu}
$$


From the first of formulae (105) and from the Kepler equation one can derive:

$$
\frac{\partial M}{\partial E}=\frac{1-e^{2}}{1+e \cos \nu}
$$

Together, (106) and (107) entail:

$$
\frac{\partial M}{\partial \nu}=\frac{\partial M}{\partial E} \frac{\partial E}{\partial \nu}=\frac{\left(1-e^{2}\right)^{3 / 2}}{(1+e \cos \nu)^{2}}
$$

whence

$$
\frac{1}{2 \pi} \int_{0}^{2 \pi} d M=\frac{\left(1-e^{2}\right)^{3 / 2}}{2 \pi} \int_{0}^{2 \pi} \frac{d \nu}{(1+e \cos \nu)^{2}}
$$

Calculation of the integral shows that the right-hand side of the above equation is equal to unity, which means that the secular parts should be calculated through the following averaging rule:

$$
\langle\ldots\rangle \equiv \frac{\left(1-e^{2}\right)^{3 / 2}}{2 \pi} \int_{0}^{2 \pi} \cdots \frac{d \nu}{(1+e \cos \nu)^{2}}
$$

In what follows below, we try hard to squeeze the calculations as much as possible, but at the same time to leave them verifiable for the interested reader.

A.3. Calculation of $\overrightarrow{\boldsymbol{\mu}} \cdot\left(\frac{\partial \overrightarrow{\boldsymbol{f}}}{\partial a} \times \overrightarrow{\mathrm{g}}-\overrightarrow{\boldsymbol{f}} \times \frac{\partial \overrightarrow{\mathrm{g}}}{\partial a}\right)$

As evident from (94) and from the first equation of (97),

$\frac{\partial \overrightarrow{\boldsymbol{f}}}{\partial a}=\left(\frac{\partial \overrightarrow{\boldsymbol{f}}}{\partial a}\right)_{\nu}+\left(\frac{\partial \overrightarrow{\boldsymbol{f}}}{\partial \nu}\right)_{a}\left(\frac{\partial \nu}{\partial a}\right)_{t, e, M_{o}}=\frac{\overrightarrow{\boldsymbol{f}}}{a}+\frac{\partial \overrightarrow{\boldsymbol{f}}}{\partial t} \frac{\partial t}{\partial \nu}\left(\frac{\partial \nu}{\partial a}\right)_{t, e, M_{o}}=\frac{\overrightarrow{\boldsymbol{f}}}{a}+\overrightarrow{\mathbf{g}} \frac{\partial t}{\partial \nu}\left(\frac{\partial \nu}{\partial a}\right)_{t, e, M_{o}}$

and, therefore,

$$
\frac{\partial \overrightarrow{\boldsymbol{f}}}{\partial a} \times \overrightarrow{\mathrm{g}}=\frac{1}{a} \overrightarrow{\boldsymbol{f}} \times \overrightarrow{\mathrm{g}}
$$

Similarly, from (95) and the second equation of (97) it ensues that

$$
\begin{gathered}
\frac{\partial \overrightarrow{\mathbf{g}}}{\partial a}=\left(\frac{\partial \overrightarrow{\mathbf{g}}}{\partial a}\right)_{\nu}+\left(\frac{\partial \overrightarrow{\mathbf{g}}}{\partial \nu}\right)_{a}\left(\frac{\partial \nu}{\partial a}\right)_{t, e, M_{o}}= \\
-\frac{\overrightarrow{\mathbf{g}}}{2 a}+\frac{\partial \overrightarrow{\mathbf{g}}}{\partial t} \frac{\partial t}{\partial \nu}\left(\frac{\partial \nu}{\partial a}\right)_{t, e, M_{o}}=-\frac{\overrightarrow{\mathbf{g}}}{2 a}+\left(-\frac{G m}{|\overrightarrow{\boldsymbol{f}}|^{3}} \overrightarrow{\boldsymbol{f}}\right) \frac{\partial t}{\partial \nu}\left(\frac{\partial \nu}{\partial a}\right)_{t, e, M_{o}}
\end{gathered}
$$


wherefrom

$$
\vec{f} \times \frac{\partial \overrightarrow{\mathbf{g}}}{\partial a}=-\frac{1}{2 a} \overrightarrow{\boldsymbol{f}} \times \overrightarrow{\mathrm{g}}
$$

In the undisturbed two-body problem, $\overrightarrow{\boldsymbol{f}} \times \overrightarrow{\mathrm{g}}$ is the angular momentum (per unit of the reduced mass) and is equal to $\sqrt{G m a\left(1-e^{2}\right)} \overrightarrow{\boldsymbol{w}}$, where the unit vector

$$
\overrightarrow{\boldsymbol{w}}=\hat{\mathbf{x}}_{1} \sin i \sin \Omega-\hat{\mathbf{x}}_{2} \sin i \cos \Omega+\hat{\mathbf{x}}_{3} \cos i
$$

is normal to the instantaneous osculating ellipse, the unit vectors $\hat{\mathbf{x}}_{1}, \hat{\mathbf{x}}_{2}, \hat{\mathbf{x}}_{3}$ making the basis of the co-precessing coordinate system $x_{1}, x_{2}, x_{3}$ (the axes $x_{1}$ and $x_{2}$ belonging to the planet's equatorial plane).

Together, (111) and (113) will give:

$$
\begin{aligned}
& \frac{\partial \overrightarrow{\boldsymbol{f}}}{\partial a} \times \overrightarrow{\mathbf{g}}-\overrightarrow{\boldsymbol{f}} \times \frac{\partial \overrightarrow{\mathbf{g}}}{\partial a}=\frac{3}{2 a} \overrightarrow{\boldsymbol{f}} \times \overrightarrow{\mathbf{g}}=\frac{3}{2 a} \sqrt{G m a\left(1-e^{2}\right)} \overrightarrow{\boldsymbol{w}} \\
= & \frac{3}{2} \sqrt{\frac{G m\left(1-e^{2}\right)}{a}}\left[\hat{\mathbf{x}}_{1} \sin i \sin \Omega-\hat{\mathbf{x}}_{2} \sin i \cos \Omega+\hat{\mathbf{x}}_{3} \cos i\right]
\end{aligned}
$$

and, thereby,

$$
\overrightarrow{\boldsymbol{\mu}} \cdot\left(\frac{\partial \overrightarrow{\boldsymbol{f}}}{\partial a} \times \overrightarrow{\mathbf{g}}-\overrightarrow{\boldsymbol{f}} \times \frac{\partial \overrightarrow{\mathbf{g}}}{\partial a}\right)=\frac{3}{2} \mu_{\perp} \sqrt{\frac{G m\left(1-e^{2}\right)}{a}}
$$

where

$$
\mu_{\perp} \equiv \mu_{1} \sin i \sin \Omega-\mu_{2} \sin i \cos \Omega+\mu_{3} \cos i
$$

Since, for constant $\overrightarrow{\boldsymbol{\mu}},(\underline{116})$ is $\nu$-independent, then in the uniform-precession case it will coincide with its orbital average.

A.4. Calculation of $\overrightarrow{\boldsymbol{\mu}} \cdot\left(\frac{\partial \overrightarrow{\boldsymbol{f}}}{\partial e} \times \overrightarrow{\mathrm{g}}-\overrightarrow{\boldsymbol{f}} \times \frac{\partial \overrightarrow{\mathbf{g}}}{\partial e}\right)$

Just as in the preceding subsection, one can write:

$$
\begin{gathered}
\frac{\partial \overrightarrow{\boldsymbol{f}}}{\partial e}=\left(\frac{\partial \overrightarrow{\boldsymbol{f}}}{\partial e}\right)_{\nu}+\left(\frac{\partial \overrightarrow{\boldsymbol{f}}}{\partial \nu}\right)_{e}\left(\frac{\partial \nu}{\partial e}\right)_{t, a, M_{o}}=\left(\frac{\partial \overrightarrow{\boldsymbol{f}}}{\partial e}\right)_{\nu}+\overrightarrow{\mathrm{g}} \frac{\partial t}{\partial \nu}\left(\frac{\partial \nu}{\partial e}\right)_{t, a, M_{o}} \\
=-\frac{2 e+\cos \nu+e^{2} \cos \nu}{(1+e \cos \nu)\left(1-e^{2}\right)} \overrightarrow{\boldsymbol{f}}+\overrightarrow{\mathrm{g}} \frac{\partial t}{\partial \nu}\left(\frac{\partial \nu}{\partial e}\right)_{t, a, M_{o}}
\end{gathered}
$$


whence

$$
\frac{\partial \overrightarrow{\boldsymbol{f}}}{\partial e} \times \overrightarrow{\mathbf{g}}=-\frac{2 e+\cos \nu+e^{2} \cos \nu}{(1+e \cos \nu)\left(1-e^{2}\right)} \overrightarrow{\boldsymbol{f}} \times \overrightarrow{\mathbf{g}}
$$

With (95) taken into account, the derivative of the two-body velocity will take the form:

$$
\begin{array}{r}
\frac{\partial \overrightarrow{\mathbf{g}}}{\partial e}=\left(\frac{\partial \overrightarrow{\mathbf{g}}}{\partial e}\right)_{\nu}+\left(\frac{\partial \overrightarrow{\mathbf{g}}}{\partial \nu}\right)_{e}\left(\frac{\partial \nu}{\partial e}\right)_{t, a, M_{o}}=\frac{e}{1-e^{2}} \overrightarrow{\mathbf{g}}+\overrightarrow{\mathbf{b}}+\frac{\partial \overrightarrow{\mathbf{g}}}{\partial t} \frac{\partial t}{\partial \nu}\left(\frac{\partial \nu}{\partial e}\right)_{t, a, M_{o}}= \\
\frac{e}{1-e^{2}} \overrightarrow{\mathbf{g}}+\overrightarrow{\mathbf{b}}+\left(-\frac{G m}{|\overrightarrow{\boldsymbol{f}}|^{3}} \overrightarrow{\boldsymbol{f}}\right) \frac{\partial t}{\partial \nu}\left(\frac{\partial \nu}{\partial e}\right)_{t, a, M_{o}}
\end{array}
$$

where

$$
\overrightarrow{\mathrm{b}} \equiv
$$

$\frac{n a}{\sqrt{1-e^{2}}}\{-\cos \Omega \sin \omega-\sin \Omega \cos \omega \cos i, \quad-\sin \Omega \sin \omega+\cos \Omega \cos \omega \cos i \quad, \quad \sin i \cos \omega\}^{\mathbf{T}}$.

From this we easily get, with aid of (114):

$$
\overrightarrow{\boldsymbol{f}} \times \overrightarrow{\mathbf{b}}=\frac{n a^{2} \sqrt{1-e^{2}}}{1+e \cos \nu}\{\sin \Omega \sin i \cos \nu, \quad-\cos \Omega \sin i \cos \nu \quad, \quad \cos i \cos \nu\}^{\mathbf{T}}=
$$

$\frac{n a^{2} \sqrt{1-e^{2}}}{1+e \cos \nu} \cos \nu \quad\{\sin i \cos \Omega, \quad-\sin i \cos \Omega, \quad \cos i\}^{\mathbf{T}}=\overrightarrow{\boldsymbol{w}} \frac{n a^{2} \sqrt{1-e^{2}}}{1+e \cos \nu} \cos \nu$.

Together, (119), (120), and (122) yield:

$$
\begin{aligned}
& \frac{\partial \overrightarrow{\boldsymbol{f}}}{\partial e} \times \overrightarrow{\mathrm{g}}-\overrightarrow{\boldsymbol{f}} \times \frac{\partial \overrightarrow{\mathbf{g}}}{\partial e}=\left[-\frac{2 e+\cos \nu+e^{2} \cos \nu}{(1+e \cos \nu)\left(1-e^{2}\right)}-\frac{e}{1-e^{2}}\right] \overrightarrow{\boldsymbol{f}} \times \overrightarrow{\mathbf{g}}-\overrightarrow{\boldsymbol{f}} \times \overrightarrow{\mathbf{b}}= \\
& -\overrightarrow{\boldsymbol{w}} \frac{3 e+\cos \nu+2 e^{2} \cos \nu}{(1+e \cos \nu)\left(1-e^{2}\right)} \sqrt{G m a\left(1-e^{2}\right)}-\overrightarrow{\boldsymbol{w}} \frac{n a^{2} \sqrt{1-e^{2}}}{1+e \cos \nu} \cos \nu= \\
& -\overrightarrow{\boldsymbol{w}} \frac{n a^{2}\left(3 e+2 \cos \nu+e^{2} \cos \nu\right)}{(1+e \cos \nu) \sqrt{1-e^{2}}},
\end{aligned}
$$

whence

$$
\overrightarrow{\boldsymbol{\mu}} \cdot\left(\frac{\partial \overrightarrow{\boldsymbol{f}}}{\partial e} \times \overrightarrow{\mathbf{g}}-\overrightarrow{\boldsymbol{f}} \times \frac{\partial \overrightarrow{\mathbf{g}}}{\partial e}\right)=-\mu_{\perp} \frac{n a^{2}\left(3 e+2 \cos \nu+e^{2} \cos \nu\right)}{(1+e \cos \nu) \sqrt{1-e^{2}}} .
$$

With aid of integrals

$$
\int_{0}^{2 \pi} \frac{d \nu}{(1+e \cos \nu)^{3}}=\pi \frac{2+e^{2}}{\left(1-e^{2}\right)^{5 / 2}}
$$


and

$$
\int_{0}^{2 \pi} \frac{\cos \nu d \nu}{(1+e \cos \nu)^{3}}=\pi \frac{-3 e}{\left(1-e^{2}\right)^{5 / 2}}
$$

it is easy to demonstrate that, under the assumption of constant $\overrightarrow{\boldsymbol{\mu}}$, the orbital average of (124) is nil.

A.5. Calculation of $\overrightarrow{\boldsymbol{\mu}} \cdot\left(\frac{\partial \overrightarrow{\boldsymbol{f}}}{\partial \omega} \times \overrightarrow{\mathrm{g}}-\overrightarrow{\boldsymbol{f}} \times \frac{\partial \overrightarrow{\mathbf{g}}}{\partial \omega}\right)$

A straightforward calculation, based on formulae (94), (95), (97), and (98), gives:

$$
\begin{gathered}
\left(\frac{\partial \overrightarrow{\boldsymbol{f}}}{\partial \omega} \times \overrightarrow{\mathbf{g}}\right)_{1}=\frac{\partial f_{2}}{\partial \omega} g_{3}-\frac{\partial f_{3}}{\partial \omega} g_{2}=-\frac{n a^{2} \sqrt{1-e^{2}}}{1+e \cos \nu} e \sin \Omega \sin i \sin \nu \\
\left(\frac{\partial \overrightarrow{\boldsymbol{f}}}{\partial \omega} \times \overrightarrow{\mathbf{g}}\right)_{2}=\frac{\partial f_{3}}{\partial \omega} g_{1}-\frac{\partial f_{1}}{\partial \omega} g_{3}=\frac{n a^{2} \sqrt{1-e^{2}}}{1+e \cos \nu} e \cos \Omega \sin i \sin \nu \\
\left(\frac{\partial \overrightarrow{\boldsymbol{f}}}{\partial \omega} \times \overrightarrow{\mathbf{g}}\right)_{3}=\frac{\partial f_{1}}{\partial \omega} g_{2}-\frac{\partial f_{2}}{\partial \omega} g_{1}=-\frac{n a^{2} \sqrt{1-e^{2}}}{1+e \cos \nu} e \cos i \sin \nu
\end{gathered}
$$

The two-body angular momentum cannot depend on the argument of the pericentre,

$$
\frac{\partial}{\partial \omega}(\overrightarrow{\boldsymbol{f}} \times \overrightarrow{\mathbf{g}})=0
$$

i.e.,

$$
\left(\overrightarrow{\boldsymbol{f}} \times \frac{\partial \overrightarrow{\mathbf{g}}}{\partial \omega}\right)_{j}=-\left(\frac{\partial \overrightarrow{\boldsymbol{f}}}{\partial \omega} \times \overrightarrow{\mathbf{g}}\right)_{j}
$$

whereby

$$
\begin{aligned}
& \left(\frac{\partial \overrightarrow{\boldsymbol{f}}}{\partial \omega} \times \overrightarrow{\mathbf{g}}-\overrightarrow{\boldsymbol{f}} \times \frac{\partial \overrightarrow{\mathbf{g}}}{\partial \omega}\right)_{1}=-2 \frac{n a^{2} \sqrt{1-e^{2}}}{1+e \cos \nu} e \sin \Omega \sin i \sin \nu, \\
& \left(\frac{\partial \overrightarrow{\boldsymbol{f}}}{\partial \omega} \times \overrightarrow{\mathbf{g}}-\overrightarrow{\boldsymbol{f}} \times \frac{\partial \overrightarrow{\mathbf{g}}}{\partial \omega}\right)_{2}=2 \frac{n a^{2} \sqrt{1-e^{2}}}{1+e \cos \nu} e \cos \Omega \sin i \sin \nu, \\
& \left(\frac{\partial \overrightarrow{\boldsymbol{f}}}{\partial \omega} \times \overrightarrow{\mathbf{g}}-\overrightarrow{\boldsymbol{f}} \times \frac{\partial \overrightarrow{\mathbf{g}}}{\partial \omega}\right)_{3}=-2 \frac{n a^{2} \sqrt{1-e^{2}}}{1+e \cos \nu} e \cos i \sin \nu .
\end{aligned}
$$


Altogether, these formulae may be written down as:

$$
\left(\frac{\partial \overrightarrow{\boldsymbol{f}}}{\partial \omega} \times \overrightarrow{\mathbf{g}}-\overrightarrow{\boldsymbol{f}} \times \frac{\partial \overrightarrow{\mathbf{g}}}{\partial \omega}\right)=-2 \overrightarrow{\boldsymbol{w}} \frac{n a^{2} \sqrt{1-e^{2}}}{1+e \cos \nu} e \sin \nu,
$$

$\overrightarrow{\boldsymbol{w}}$ being the unit vector pointing in the direction of the angular momentum. It is given by (114). Finally,

$$
\overrightarrow{\boldsymbol{\mu}} \cdot\left(\frac{\partial \overrightarrow{\boldsymbol{f}}}{\partial \omega} \times \overrightarrow{\mathbf{g}}-\overrightarrow{\boldsymbol{f}} \times \frac{\partial \overrightarrow{\mathbf{g}}}{\partial \omega}\right)=-2 \mu_{\perp} \frac{n a^{2} \sqrt{1-e^{2}}}{1+e \cos \nu} e \sin \nu .
$$

Since $\sin \nu$ is odd and $(1+e \cos \nu)^{-3}$ is even, it is obvious that, for a constant $\overrightarrow{\boldsymbol{\mu}}$, the orbital average of (136) will vanish.

\section{A.6. Calculation of $\overrightarrow{\boldsymbol{\mu}} \cdot\left(\frac{\partial \overrightarrow{\boldsymbol{f}}}{\partial \Omega} \times \overrightarrow{\mathrm{g}}-\overrightarrow{\boldsymbol{f}} \times \frac{\partial \overrightarrow{\mathbf{g}}}{\partial \Omega}\right)$.}

Once again, formulae (94), (95), (97), and (98) yield:

$$
\begin{aligned}
& \left(\frac{\partial \overrightarrow{\boldsymbol{f}}}{\partial \Omega} \times \overrightarrow{\mathbf{g}}\right)_{1}=\frac{\partial f_{2}}{\partial \Omega} g_{3}-\frac{\partial f_{3}}{\partial \Omega} g_{2}= \\
& \frac{n a^{2} \sqrt{1-e^{2}}}{1+e \cos \nu}[\cos \Omega \cos (\omega+\nu)-\sin \Omega \sin (\omega+\nu) \cos i] \cos (\omega+\nu) \sin i \\
& +\frac{n a^{2} \sqrt{1-e^{2}}}{1+e \cos n u} e[\cos \Omega \cos (\omega+\nu)-\sin \Omega \sin (\omega+\nu) \cos i] \cos \omega \sin i, \\
& \left(\frac{\partial \overrightarrow{\boldsymbol{f}}}{\partial \Omega} \times \overrightarrow{\mathbf{g}}\right)_{2}=\frac{\partial f_{3}}{\partial \Omega} g_{1}-\frac{\partial f_{1}}{\partial \Omega} g_{3}= \\
& \frac{n a^{2} \sqrt{1-e^{2}}}{1+e \cos \nu}[\sin \Omega \cos (\omega+\nu)+\cos \Omega \sin (\omega+\nu) \cos i] \cos (\omega+\nu) \sin i \\
& +\frac{n a^{2} \sqrt{1-e^{2}}}{1+e \cos \nu} e[\sin \Omega \cos (\omega+\nu)+\cos \Omega \sin (\omega+\nu) \cos i] \cos \omega \sin i, \\
& \left(\frac{\partial \overrightarrow{\boldsymbol{f}}}{\partial \Omega} \times \overrightarrow{\mathbf{g}}\right)_{3}=\frac{\partial f_{1}}{\partial \Omega} g_{2}-\frac{\partial f_{2}}{\partial \Omega} g_{1}=
\end{aligned}
$$




$$
\begin{aligned}
& \frac{n a^{2} \sqrt{1-e^{2}}}{1+e \cos \nu}\{[-\sin \Omega \cos (\omega+\nu) \\
& -\cos \Omega \sin (\omega+\nu) \cos i] \quad[-\sin \Omega \sin (\omega+\nu)+\cos \Omega \cos i \cos (\omega+\nu)]- \\
& [\cos \Omega \cos (\omega+\nu)-\sin \Omega \sin (\omega+\nu) \cos i][-\cos \Omega \sin (\omega+\nu)-\sin \Omega \cos i \cos (\omega+\nu)]\} \\
& +\frac{n a^{2} \sqrt{1-e^{2}}}{1+e \cos \nu} e\{[-\sin \Omega \cos (\omega+\nu)- \\
& \cos \Omega \sin (\omega+\nu) \cos i][-\sin \Omega \sin \omega+\cos \Omega \cos i \cos \omega] \\
& -[\cos \Omega \cos (\omega+\nu)-\sin \Omega \sin (\omega+\nu) \cos i][-\cos \Omega \sin \omega-\sin \Omega \cos i \cos \omega]\}= \\
& \frac{n a^{2} \sqrt{1-e^{2}}}{1+e \cos \nu}\left\{\sin (\omega+\nu) \cos (\omega+\nu) \sin ^{2} i+e\left[\sin \omega \cos (\omega+\nu)-\sin (\omega+\nu) \cos \omega \cos ^{2} i\right]\right\} \\
& \left(\overrightarrow{\boldsymbol{f}} \times \frac{\partial \overrightarrow{\mathbf{g}}}{\partial \Omega}\right)_{1}=f_{2} \frac{\partial \overrightarrow{\mathbf{g}}_{3}}{\partial \Omega}-f_{3} \frac{\partial \overrightarrow{\mathbf{g}}_{2}}{\partial \Omega}= \\
& \frac{n a^{2} \sqrt{1-e^{2}}}{1+e \cos \nu} \quad[-\sin (\omega+\nu) \sin i] \quad[-\cos \Omega \sin (\omega+\nu)-\sin \Omega \cos (\omega+\nu) \cos i] \\
& +\frac{n a^{2} \sqrt{1-e^{2}}}{1+e \cos \nu} e[-\sin (\omega+\nu) \sin i] \quad[-\cos \Omega \sin \omega-\sin \Omega \cos \omega \cos i] \\
& =\frac{n a^{2} \sqrt{1-e^{2}}}{1+e \cos \nu} \sin i\left[\sin ^{2}(\omega+\nu) \cos \Omega+\sin (\omega+\nu) \cos (\omega+\nu) \sin \Omega \cos i\right] \\
& +\frac{n a^{2} \sqrt{1-e^{2}}}{1+e \cos n u} \quad e \sin i \quad[\sin (\omega+\nu) \sin \omega \cos \Omega+\sin (\omega+\nu) \cos \omega \sin \Omega \cos i]
\end{aligned}
$$




$$
\begin{aligned}
& \left(\overrightarrow{\boldsymbol{f}} \times \frac{\partial \overrightarrow{\mathbf{g}}}{\partial \Omega}\right)_{2}=f_{3} \frac{\partial \overrightarrow{\mathbf{g}}_{1}}{\partial \Omega}-f_{1} \frac{\partial \overrightarrow{\mathbf{g}}_{3}}{\partial \Omega}= \\
& \frac{n a^{2} \sqrt{1-e^{2}}}{1+e \cos \nu} \sin (\omega+\nu) \sin i \quad[\sin \Omega \sin (\omega+\nu)-\cos \Omega \cos (\omega+\nu) \cos i] \\
& +\frac{n a^{2} \sqrt{1-e^{2}}}{1+e \cos \nu} \quad e \sin (\omega+\nu) \sin i \quad[\sin \Omega \sin \omega-\cos \Omega \cos \omega \cos i], \\
& \left(\frac{\partial \overrightarrow{\boldsymbol{f}}}{\partial \Omega} \times \overrightarrow{\mathbf{g}}\right)_{3}=\frac{\partial f_{1}}{\partial \Omega} g_{2}-\frac{\partial f_{2}}{\partial \Omega} g_{1}= \\
& \frac{n a^{2} \sqrt{1-e^{2}}}{1+e \cos \nu}\{[\cos \Omega \cos (\omega+\nu) \\
& -\sin \Omega \sin (\omega+\nu) \cos i] \quad[-\cos \Omega \sin (\omega+\nu)-\sin \Omega \cos i \cos (\omega+\nu)]- \\
& [\sin \Omega \cos (\omega+\nu)+\cos \Omega \sin (\omega+\nu) \cos i] \quad[\sin \Omega \sin (\omega+\nu)-\cos \Omega \cos i \cos (\omega+\nu)]\} \\
& +\frac{n a^{2} \sqrt{1-e^{2}}}{1+e \cos \nu} e\{[\cos \Omega \cos (\omega+\nu)- \\
& \sin \Omega \sin (\omega+\nu) \cos i][-\cos \Omega \sin \omega-\sin \Omega \cos i \cos \omega] \\
& -[\sin \Omega \cos (\omega+\nu)+\cos \Omega \sin (\omega+\nu) \cos i][\sin \Omega \sin \omega-\cos \Omega \cos i \cos \omega]\}=
\end{aligned}
$$$$
\frac{n a^{2} \sqrt{1-e^{2}}}{1+e \cos \nu}\left\{-\sin (\omega+\nu) \cos (\omega+\nu) \sin ^{2} i+e\left[-\sin \omega \cos (\omega+\nu)+\sin (\omega+\nu) \cos \omega \cos ^{2} i\right]\right\}
$$$$
\text { In (137- 138) and in (140-141) we benefitted from } f_{3} \text { being independent of } \Omega \text {. Now, by }
$$ 
subtracting, accordingly, (140) from (137), and (141) from (138), and (142) from (139), we arrive, after some intermediate algebra, at the following three expressions:

$$
\begin{aligned}
& \left(\frac{\partial \overrightarrow{\boldsymbol{f}}}{\partial \Omega} \times \overrightarrow{\mathbf{g}}-\overrightarrow{\boldsymbol{f}} \times \frac{\partial \overrightarrow{\mathbf{g}}}{\partial \Omega}\right)_{1}= \\
& \frac{n a^{2} \sqrt{1-e^{2}}}{1+e \cos \nu} \sin i \quad\{\cos \Omega \cos [2(\omega+\nu)]-\sin \Omega \cos i \sin [2(\omega+\nu)]\} \\
& +\frac{n a^{2} \sqrt{1-e^{2}}}{1+e \cos \nu} e \sin i\{\cos \Omega \cos (\nu+2 \omega)-2 \sin \Omega \cos i \sin (\omega+\nu) \cos \omega\}, \\
& \left(\frac{\partial \overrightarrow{\boldsymbol{f}}}{\partial \Omega} \times \overrightarrow{\mathbf{g}}-\overrightarrow{\boldsymbol{f}} \times \frac{\partial \overrightarrow{\mathbf{g}}}{\partial \Omega}\right)_{2}= \\
& \frac{n a^{2} \sqrt{1-e^{2}}}{1+e \cos \nu} \sin i \quad\{\sin \Omega \cos [2(\omega+\nu)]+\cos \Omega \cos i \sin [2(\omega+\nu)]\} \\
& +\frac{n a^{2} \sqrt{1-e^{2}}}{1+e \cos \nu} e \sin i\{\sin \Omega \cos (\nu+2 \omega)+2 \cos \Omega \cos i \sin (\omega+\nu) \cos \omega\} \\
& \left(\frac{\partial \overrightarrow{\boldsymbol{f}}}{\partial \Omega} \times \overrightarrow{\mathbf{g}}-\overrightarrow{\boldsymbol{f}} \times \frac{\partial \overrightarrow{\mathbf{g}}}{\partial \Omega}\right)_{3}= \\
& \frac{n a^{2} \sqrt{1-e^{2}}}{1+e \cos \nu} \sin ^{2} i \quad \sin [2(\omega+\nu)] \\
& +\frac{n a^{2} \sqrt{1-e^{2}}}{1+e \cos \nu} e\left\{2 \sin \omega \cos (\nu+\omega)-2 \sin (\omega+\nu) \cos \omega \cos ^{2} i\right\}= \\
& \frac{n a^{2} \sqrt{1-e^{2}}}{1+e \cos \nu} \sin ^{2} i \sin [2(\omega+\nu)]+\frac{n a^{2} \sqrt{1-e^{2}}}{1+e \cos \nu} 2 e\left\{-\sin \nu+\sin ^{2} i \cos \omega \sin (\omega+\nu)\right\} .
\end{aligned}
$$

Hence, the overall expression for $\overrightarrow{\boldsymbol{\mu}} \cdot((\partial \overrightarrow{\boldsymbol{f}} / \partial \Omega) \times \overrightarrow{\mathbf{g}}-\overrightarrow{\boldsymbol{f}} \times(\partial \overrightarrow{\mathbf{g}} / \partial \Omega))$ will be given by (78). To average this expression, in the simple case of a constant $\overrightarrow{\boldsymbol{\mu}}$, it will be sufficient to separately average the above three expressions (143 - 145) and then to multiply the 
averages by $\mu_{1}, \mu_{2}$ and $\mu_{3}$, correspondingly, and to add everything up. To carry out this procedure, it will be convenient first to regroup terms in (143-145) and to through out the inputs proportional to the odd functions $\sin \nu$ and $\sin 2 \nu$, because these inputs will vanish after averaging via formula (109). Thus we get:

$\left\langle\left(\frac{\partial \overrightarrow{\boldsymbol{f}}}{\partial \Omega} \times \overrightarrow{\mathbf{g}}-\overrightarrow{\boldsymbol{f}} \times \frac{\partial \overrightarrow{\mathbf{g}}}{\partial \Omega}\right)_{1}\right\rangle=$

$\left\langle\frac{n a^{2} \sqrt{1-e^{2}}}{1+e \cos \nu} \sin i\{\cos \Omega \cos 2 \omega \cos 2 \nu-\cos \Omega \sin 2 \omega \sin 2 \nu\right.$

$-\sin \Omega \cos i \sin 2 \omega \cos 2 \nu+\sin \Omega \cos i \cos 2 \omega \sin 2 \nu\}$

$+\frac{n a^{2} \sqrt{1-e^{2}}}{1+e \cos \nu} e \sin i\{\cos \Omega \cos 2 \omega \cos \nu-\cos \Omega \sin 2 \omega \sin \nu$

$\left.\left.-\sin \Omega \cos i \sin 2 \omega \cos \nu+2 \sin \Omega \cos i \cos ^{2} \omega \sin \nu\right\}\right\rangle=$

$\left\langle\frac{n a^{2} \sqrt{1-e^{2}}}{1+e \cos \nu} \sin i(\cos \Omega \cos 2 \omega-\sin \Omega \cos i \sin 2 \omega)(\cos 2 \nu+e \cos \nu)\right\rangle=$

$\frac{\left(1-e^{2}\right)^{3 / 2}}{2 \pi} n a^{2} \sqrt{1-e^{2}} \sin i(\cos \Omega \cos 2 \omega-\sin \Omega \cos i \sin 2 \omega) \int_{0}^{2 \pi} \frac{\cos 2 \nu+e \cos \nu}{(1+e \cos \nu)^{3}} d \nu=0$

$\left\langle\left(\frac{\partial \overrightarrow{\boldsymbol{f}}}{\partial \Omega} \times \overrightarrow{\mathbf{g}}-\overrightarrow{\boldsymbol{f}} \times \frac{\partial \overrightarrow{\mathbf{g}}}{\partial \Omega}\right)_{2}\right\rangle=$

$\left\langle\frac{n a^{2} \sqrt{1-e^{2}}}{1+e \cos \nu} \sin i\{\sin \Omega \cos 2 \omega \cos 2 \nu-\sin \Omega \sin 2 \omega \sin 2 \nu\right.$

$+\cos \Omega \cos i \sin 2 \omega \cos 2 \nu+\cos \Omega \cos i \cos 2 \omega \sin 2 \nu\}$ 


$$
\begin{aligned}
& +\frac{n a^{2} \sqrt{1-e^{2}}}{1+e \cos \nu} e \sin i\{\sin \Omega \cos 2 \omega \cos \nu-\sin \Omega \sin 2 \omega \sin \nu \\
& \left.\left.+\cos \Omega \cos i \sin 2 \omega \cos \nu+2 \cos \Omega \cos i \cos ^{2} \omega \sin \nu\right\}\right\rangle= \\
& \left\langle\frac{n a^{2} \sqrt{1-e^{2}}}{1+e \cos \nu} \sin i(\sin \Omega \cos 2 \omega+\cos \Omega \cos i \sin 2 \omega)(\cos 2 \nu+e \cos \nu)\right\rangle= \\
& \frac{\left(1-e^{2}\right)^{3 / 2}}{2 \pi} n a^{2} \sqrt{1-e^{2}} \sin i(\sin \Omega \cos 2 \omega+\cos \Omega \cos i \sin 2 \omega) \int_{0}^{2 \pi} \frac{\cos 2 \nu+e \cos \nu}{(1+e \cos \nu)^{3}} d \nu=0 \\
& \left\langle\left(\frac{\partial \overrightarrow{\boldsymbol{f}}}{\partial \Omega} \times \overrightarrow{\mathbf{g}}-\overrightarrow{\boldsymbol{f}} \times \frac{\partial \overrightarrow{\mathbf{g}}}{\partial \Omega}\right)_{3}\right\rangle= \\
& \left\langle\frac{n a^{2} \sqrt{1-e^{2}}}{1+e \cos \nu} \sin ^{2} i(\sin 2 \omega \cos 2 \nu+\cos 2 \omega \sin 2 \nu)+\right. \\
& \left.\frac{n a^{2} \sqrt{1-e^{2}}}{1+e \cos \nu} 2 e\left(-\sin \nu+\sin ^{2} i \cos \omega \sin \omega \cos \nu+\sin ^{2} i \cos ^{2} \omega \sin \nu\right)\right\rangle= \\
& \left\langle\frac{n a^{2} \sqrt{1-e^{2}}}{1+e \cos \nu} \sin ^{2} i \sin 2 \omega(\cos 2 \nu+e \cos \nu)\right\rangle= \\
& \frac{\left(1-e^{2}\right)^{3 / 2}}{2 \pi} n a^{2} \sqrt{1-e^{2}} \sin ^{2} i \sin 2 \omega \int_{0}^{2 \pi} \frac{\cos 2 \nu+e \cos \nu}{(1+e \cos \nu)^{3}} d \nu=0
\end{aligned}
$$

We see that the averages of all three Cartesian components of $((\partial \overrightarrow{\boldsymbol{f}} / \partial \Omega) \times \overrightarrow{\mathbf{g}}-\overrightarrow{\boldsymbol{f}} \times(\partial \overrightarrow{\mathbf{g}} / \partial \Omega))$ vanish. In the developments (146-148) the following integrals were used:

$$
\int_{0}^{2 \pi} \frac{\cos \nu}{(1+e \cos \nu)^{3}}=-\sqrt{\frac{1-e}{1+e}} \frac{3 \pi e}{(1-e)^{3}(1+e)^{2}}
$$

and

$$
\int_{0}^{2 \pi} \frac{\cos 2 \nu}{(1+e \cos \nu)^{3}}=\sqrt{\frac{1-e}{1+e}} \frac{3 \pi e^{2}}{(1-e)^{3}(1+e)^{2}} .
$$


A.7. Calculation of $\overrightarrow{\boldsymbol{\mu}} \cdot\left(\frac{\partial \overrightarrow{\boldsymbol{f}}}{\partial i} \times \overrightarrow{\mathrm{g}}-\overrightarrow{\boldsymbol{f}} \times \frac{\partial \overrightarrow{\mathbf{g}}}{\partial i}\right)$

Just like the preceding subsections, this one is based on formulae (94), (95), (97), and (98):

$$
\begin{aligned}
& \left(\frac{\partial \overrightarrow{\boldsymbol{f}}}{\partial i} \times \overrightarrow{\mathbf{g}}\right)_{1}=\frac{\partial f_{2}}{\partial i} g_{3}-\frac{\partial f_{3}}{\partial i} g_{2}= \\
& \frac{n a^{2} \sqrt{1-e^{2}}}{1+e \cos \nu}\{[-\sin (\omega+\nu) \sin i \cos \Omega][\sin i \cos (\omega+\nu)]-
\end{aligned}
$$

$$
[\cos i \sin (\omega+\nu)][-\sin \Omega \sin (\omega+\nu)+\cos \Omega \cos (\omega+\nu) \cos i]\}
$$

$+\frac{n a^{2} \sqrt{1-e^{2}}}{1+e \cos \nu} e\{[-\sin (\omega+\nu) \sin i \cos \Omega][\sin i \cos \omega]-$

$$
[\cos i \sin (\omega+\nu)][-\sin \Omega \sin \omega+\cos \Omega \cos \omega \cos i]\}=
$$

$$
\frac{n a^{2} \sqrt{1-e^{2}}}{1+e \cos \nu}\left\{\left[\sin \Omega \sin ^{2}(\omega+\nu) \cos i-\cos \Omega \sin (\omega+\nu) \cos (\omega+\nu)\right]+\right.
$$

$e \sin (\omega+\nu)[\sin \Omega \sin \omega \cos i-\cos \Omega \cos \omega]\}$,

$$
\left(\frac{\partial \overrightarrow{\boldsymbol{f}}}{\partial i} \times \overrightarrow{\mathrm{g}}\right)_{2}=\frac{\partial f_{3}}{\partial i} g_{1}-\frac{\partial f_{1}}{\partial i} g_{3}=
$$

$$
\frac{n a^{2} \sqrt{1-e^{2}}}{1+e \cos \nu}\{[\sin (\omega+\nu) \cos i][-\cos \Omega \sin (\omega+\nu)-\sin \Omega \cos i \cos (\omega+\nu)]-
$$




$$
[\sin \Omega \sin i \sin (\omega+\nu)][\sin i \cos (\omega+\nu)]\}
$$

$+\frac{n a^{2} \sqrt{1-e^{2}}}{1+e \cos n u} e\{[\sin (\omega+\nu) \cos i][-\cos \Omega \sin \omega-\sin \Omega \cos \omega \cos i]-$

$[\sin \Omega \sin (\omega+\nu) \sin i] \quad[\sin i \cos \omega]\}=$

$$
\begin{gathered}
\frac{n a^{2} \sqrt{1-e^{2}}}{1+e \cos \nu}\left\{\left[-\sin \Omega \sin (\omega+\nu) \cos (\omega+\nu)-\cos \Omega \sin ^{2}(\omega+\nu) \cos i\right]+\right. \\
e[-\sin \Omega \sin (\omega+\nu) \cos \omega-\cos \Omega \cos i \sin \omega \sin (\omega+\nu)]\} \\
\left(\frac{\partial \overrightarrow{\boldsymbol{f}}}{\partial i} \times \overrightarrow{\mathbf{g}}\right)_{3}=\frac{\partial f_{1}}{\partial i} g_{2}-\frac{\partial f_{2}}{\partial i} g_{1}=
\end{gathered}
$$

$\frac{n a^{2} \sqrt{1-e^{2}}}{1+e \cos \nu}\{[\sin (\omega+\nu) \sin i \sin \Omega][-\sin \Omega \sin (\omega+\nu)+\cos \Omega \cos i \cos (\omega+\nu)]-$

$$
[-\cos \Omega \sin (\omega+\nu) \sin i] \quad[-\cos \Omega \sin (\omega+\nu)-\sin \Omega \cos (\omega+\nu) \cos i]\}
$$

$+\frac{n a^{2} \sqrt{1-e^{2}}}{1+e \cos \nu} e\{[\sin (\omega+\nu) \sin i \sin \Omega][-\sin \Omega \sin \omega+\cos \Omega \cos i \cos \omega]-$

$$
[-\cos \Omega \sin (\omega+\nu) \sin i] \quad[-\cos \Omega \sin \omega-\sin \Omega \cos \omega \cos i]\}=
$$

$$
\frac{n a^{2} \sqrt{1-e^{2}}}{1+e \cos \nu}\left\{-\sin ^{2}(\omega+\nu) \sin i-e \sin (\omega+\nu) \sin \omega \sin i\right\}
$$

$$
\left(\overrightarrow{\boldsymbol{f}} \times \frac{\partial \overrightarrow{\mathbf{g}}}{\partial i}\right)_{1}=f_{2} \frac{\partial g_{3}}{\partial i}-f_{3} \frac{\partial g_{2}}{\partial i}=
$$




$$
\begin{aligned}
& \frac{n a^{2} \sqrt{1-e^{2}}}{1+e \cos \nu}\{[\sin \Omega \cos (\omega+\nu)+\cos \Omega \sin (\omega+\nu) \cos i] \cos (\omega+\nu) \cos i- \\
& \sin (\omega+\nu) \sin i \quad[-\cos \Omega \cos (\omega+\nu) \sin i]\} \\
& +\frac{n a^{2} \sqrt{1-e^{2}}}{1+e \cos \nu} e\{[\sin \Omega \cos (\omega+\nu)+\cos \Omega \sin (\omega+\nu) \cos i] \cos \omega \cos i- \\
& \sin (\omega+\nu) \sin i \quad[-\cos \Omega \cos \omega \sin i]\}= \\
& \frac{n a^{2} \sqrt{1-e^{2}}}{1+e \cos \nu}\left\{\left[\cos \Omega \sin (\omega+\nu) \cos (\omega+\nu)+\sin \Omega \cos ^{2}(\omega+\nu) \cos i\right]+\right. \\
& e[\cos \Omega \sin (\omega+\nu) \cos \omega+\sin \Omega \cos (\omega+\nu) \cos \omega \cos i]\}, \\
& \left(\overrightarrow{\boldsymbol{f}} \times \frac{\partial \overrightarrow{\mathbf{g}}}{\partial i}\right)_{2}=f_{3} \frac{\partial g_{1}}{\partial i}-f_{1} \frac{\partial g_{3}}{\partial i}= \\
& \frac{n a^{2} \sqrt{1-e^{2}}}{1+e \cos \nu}\{\sin (\omega+\nu) \sin i \sin \Omega \sin i \cos (\omega+\nu)- \\
& [\cos \Omega \cos (\omega+\nu)-\sin \Omega \sin (\omega+\nu) \cos i] \cos (\omega+\nu) \cos i\} \\
& +\frac{n a^{2} \sqrt{1-e^{2}}}{1+e \cos \nu} e\{\sin (\omega+\nu) \sin i \sin \Omega \sin i \cos \omega- \\
& [\cos \Omega \cos (\omega+\nu)-\sin \Omega \sin (\omega+\nu) \cos i] \cos \omega \cos i\}= \\
& \frac{n a^{2} \sqrt{1-e^{2}}}{1+e \cos \nu}\left\{\left[\sin \Omega \sin (\omega+\nu) \cos (\omega+\nu)-\cos \Omega \cos ^{2}(\omega+\nu) \cos i\right]+\right.
\end{aligned}
$$




$$
\begin{aligned}
& e[\sin \Omega \sin (\omega+\nu) \cos \omega-\cos \Omega \cos (\omega+\nu) \cos \omega \cos i]\}, \\
& \left(\overrightarrow{\boldsymbol{f}} \times \frac{\partial \overrightarrow{\mathbf{g}}}{\partial i}\right)_{3}=f_{1} \frac{\partial g_{2}}{\partial i}-f_{2} \frac{\partial g_{1}}{\partial i}= \\
& \frac{n a^{2} \sqrt{1-e^{2}}}{1+e \cos \nu}\{[\cos \Omega \cos (\omega+\nu)-\sin \Omega \sin (\omega+\nu) \cos i][-\cos \Omega \sin i \cos (\omega+\nu)]- \\
& [\sin \Omega \cos (\omega+\nu)+\cos \Omega \sin (\omega+\nu) \cos i] \sin \Omega \cos (\omega+\nu) \cos i\} \\
& +\frac{n a^{2} \sqrt{1-e^{2}}}{1+e \cos \nu} e\{[\cos \Omega \cos (\omega+\nu)-\sin \Omega \sin (\omega+\nu) \cos i][-\cos \Omega \sin i \cos \omega]- \\
& [\sin \Omega \cos (\omega+\nu)+\cos \Omega \sin (\omega+\nu) \cos i] \sin \Omega \cos \omega \cos i\}= \\
& \frac{n a^{2} \sqrt{1-e^{2}}}{1+e \cos \nu}\left\{-\sin i \cos ^{2}(\omega+\nu)-e \sin i \cos (\omega+\nu) \cos \omega\right\} .
\end{aligned}
$$

Now, by putting together, accordingly, (151) with (154), (152) with (155), and (153) with (156), we arrive, after some intermediate algebra, to the following expressions for the three Cartesian components:

$$
\left(\frac{\partial \overrightarrow{\boldsymbol{f}}}{\partial i} \times \overrightarrow{\mathbf{g}}-\overrightarrow{\boldsymbol{f}} \times \frac{\partial \overrightarrow{\mathbf{g}}}{\partial i}\right)_{1}=
$$

$\frac{n a^{2} \sqrt{1-e^{2}}}{1+e \cos \nu}\left\{\left[\sin \Omega \cos i\left(\sin ^{2}(\omega+\nu)-\cos ^{2}(\omega+\nu)\right)-2 \cos \Omega \sin (\omega+\nu) \cos (\omega+\nu)\right]+\right.$

$e[\sin \Omega \cos i(-\cos (\omega+\nu) \cos \omega+\sin (\omega+\nu) \sin \omega)-2 \cos \Omega \sin (\omega+\nu) \cos \omega]\}=$

$$
\frac{n a^{2} \sqrt{1-e^{2}}}{1+e \cos \nu}\left\{\left[(-\sin \Omega \cos i \cos 2 \omega-\cos \Omega \sin 2 \omega)\left(\cos ^{2} \nu-\sin ^{2} \nu\right)+\right.\right.
$$


$2 \sin \nu \cos \nu(\sin \Omega \cos i \sin 2 \omega-\cos \Omega \cos 2 \omega)]+$

$\left.e\left[(-\sin \Omega \cos i \cos 2 \omega-\cos \Omega \sin 2 \omega) \cos \nu+\left(\sin \Omega \cos i \sin 2 \omega-2 \cos \Omega \cos ^{2} \omega\right) \sin \nu\right]\right\}$,

$$
\left(\frac{\partial \overrightarrow{\boldsymbol{f}}}{\partial i} \times \overrightarrow{\mathrm{g}}-\overrightarrow{\boldsymbol{f}} \times \frac{\partial \overrightarrow{\mathbf{g}}}{\partial i}\right)_{2}=
$$

$\frac{n a^{2} \sqrt{1-e^{2}}}{1+e \cos \nu}\left\{\left[\cos \Omega \cos i\left(\cos ^{2}(\omega+\nu)-\sin ^{2}(\omega+\nu)\right)-2 \sin \Omega \sin (\omega+\nu) \cos (\omega+\nu)\right]+\right.$

$e[\cos \Omega \cos i(\cos (\omega+\nu) \cos \omega-\sin (\omega+\nu) \sin \omega)-2 \sin \Omega \sin (\omega+\nu) \cos \omega]\}=$ $\frac{n a^{2} \sqrt{1-e^{2}}}{1+e \cos \nu}\left\{\left[(\cos \Omega \cos i \cos 2 \omega-\sin \Omega \sin 2 \omega)\left(\cos ^{2} \nu-\sin ^{2} \nu\right)+\right.\right.$ $2 \sin \nu \cos \nu(-\cos \Omega \cos i \sin 2 \omega-\sin \Omega \cos 2 \omega)]+$

$\left.e\left[(\cos \Omega \cos i \cos 2 \omega-\sin \Omega \sin 2 \omega) \cos \nu+\left(-2 \sin \Omega \cos ^{2} \omega+\cos \Omega \sin 2 \omega \cos i\right) \sin \nu\right]\right\}$,

$$
\left(\frac{\partial \overrightarrow{\boldsymbol{f}}}{\partial i} \times \overrightarrow{\mathrm{g}}-\overrightarrow{\boldsymbol{f}} \times \frac{\partial \overrightarrow{\mathbf{g}}}{\partial i}\right)_{3}=
$$

$\frac{n a^{2} \sqrt{1-e^{2}}}{1+e \cos \nu}\left\{\sin i\left[\cos ^{2}(\omega+\nu)-\sin ^{2}(\omega+\nu)\right]+\right.$

$e[\sin i \cos \omega \cos (\omega+\nu)-\sin i \sin \omega \sin (\omega+\nu)]\}=$ 
$\frac{n a^{2} \sqrt{1-e^{2}}}{1+e \cos \nu}\left\{\sin i\left[\cos 2 \omega\left(\cos ^{2} \nu-\sin ^{2} \nu\right)-2 \sin 2 \omega \sin \nu \cos \nu\right]+\right.$

$e[\sin i \cos 2 \omega \cos \nu-\sin i \sin 2 \omega \sin \nu]\}$.

Averaging of the afore calculated three Cartesian components of $((\partial \overrightarrow{\boldsymbol{f}} / \partial i) \times \overrightarrow{\mathbf{g}}-\overrightarrow{\boldsymbol{f}} \times(\partial \overrightarrow{\mathbf{g}} / \partial i))$ almost exactly coincides with averaging of $((\partial \overrightarrow{\boldsymbol{f}} / \partial \Omega) \times \overrightarrow{\mathbf{g}}-\overrightarrow{\boldsymbol{f}} \times(\partial \overrightarrow{\mathbf{g}} / \partial \Omega))$ presented in the preceding subsection, and the result is the same, nil. Indeed, as can be easily seen from (157 - 159), after we through out the (vanishing after averaging) inputs proportional to the odd functions $\sin \nu$ and $\sin 2 \nu$, each of the three expressions (157-159) will be proportional to $(\cos 2 \nu+e \cos \nu) /(1+e \cos \nu)$. Averaging of this expression via formula (109) will vanish, as can be easily seen from (149-150).

A.8. Calculation of $\overrightarrow{\boldsymbol{\mu}} \cdot\left(\frac{\partial \overrightarrow{\boldsymbol{f}}}{\partial M_{o}} \times \overrightarrow{\mathrm{g}}-\overrightarrow{\boldsymbol{f}} \times \frac{\partial \overrightarrow{\mathrm{g}}}{\partial M_{o}}\right)$.

This calculation will be the easiest: as

$$
\frac{\partial \overrightarrow{\boldsymbol{f}}}{\partial M_{o}}=\frac{\partial \overrightarrow{\boldsymbol{f}}}{\partial t} \frac{\partial t}{\partial M_{o}}=\overrightarrow{\mathbf{g}} \frac{\partial t}{\partial M_{o}}
$$

and

$$
\frac{\partial \overrightarrow{\mathbf{g}}}{\partial M_{o}}=\frac{\partial \overrightarrow{\mathbf{g}}}{\partial t} \frac{\partial t}{\partial M_{o}}=\left(-\frac{G m}{|\overrightarrow{\boldsymbol{f}}|^{3}} \overrightarrow{\boldsymbol{f}}\right) \frac{\partial t}{\partial M_{o}}
$$

then, obviously,

$$
\overrightarrow{\boldsymbol{\mu}} \cdot\left(\frac{\partial \overrightarrow{\boldsymbol{f}}}{\partial M_{o}} \times \overrightarrow{\mathbf{g}}-\overrightarrow{\boldsymbol{f}} \times \frac{\partial \overrightarrow{\mathbf{g}}}{\partial M_{o}}\right)=\overrightarrow{\boldsymbol{\mu}} \cdot(0-0)=0 .
$$

A.9. Calculation of $\dot{\overrightarrow{\boldsymbol{\mu}}} \cdot\left(-\overrightarrow{\boldsymbol{f}} \times \frac{\partial \overrightarrow{\boldsymbol{f}}}{\partial a}\right)$.

With aid of (110) and (68) we establish:

$$
\begin{gathered}
-\overrightarrow{\boldsymbol{f}} \times \frac{\partial \overrightarrow{\boldsymbol{f}}}{\partial a}=-\overrightarrow{\boldsymbol{f}} \times \overrightarrow{\mathbf{g}}\left(\frac{\partial t}{\partial E}\right)_{a, e, M_{o}}\left(\frac{\partial E}{\partial a}\right)_{t, e, M_{o}}= \\
-\overrightarrow{\mathbf{w}} \sqrt{G m a\left(1-e^{2}\right)} \frac{1}{n} \frac{1-e^{2}}{1+e \cos \nu}\left(\frac{\partial E}{\partial a}\right)_{t, e, M_{o}}=-\overrightarrow{\mathbf{w}} a^{2} \frac{\left(1-e^{2}\right)^{3 / 2}}{1+e \cos \nu}\left(\frac{\partial E}{\partial a}\right)_{t, e, M_{o}}
\end{gathered}
$$


where we used the formula

$$
\left(\frac{\partial t}{\partial E}\right)_{a, e, M_{o}}=\frac{1-e \cos E}{n}=\frac{1}{n} \frac{1-e^{2}}{1+e \cos \nu}
$$

that follows from the Kepler equation and from the first equation of (105).

It remains to compute $\partial E / \partial a$. From the Kepler equation

$$
E-e \sin E=M_{o}+n\left(t-t_{o}\right)
$$

it follows that

$$
d E(1-e \cos E)=-\frac{3}{2} n a^{-1}\left(t-t_{o}\right) d a
$$

wherefrom

$$
\begin{gathered}
\left(\frac{\partial E}{\partial a}\right)_{t, e, M_{o}}=-\frac{3}{2} a^{-1} \frac{n\left(t-t_{o}\right)}{1-e \cos E}=-\frac{3}{2} a^{-1} \frac{E-e \sin E-M_{o}}{1-e \cos E} \\
=-\frac{3}{2} n a^{-1} \frac{1+\cos \nu}{1-e^{2}}\left(t-t_{o}\right) .
\end{gathered}
$$

Insertion of the above into (163) will give:

$$
-\overrightarrow{\boldsymbol{f}} \times \frac{\partial \overrightarrow{\boldsymbol{f}}}{\partial a}=\frac{3}{2} \overrightarrow{\mathbf{w}} a n\left(t-t_{o}\right) \sqrt{1-e^{2}},
$$

expression linear in $t-t_{o}$.

\section{A.10. Calculation of $\dot{\vec{\mu}} \cdot\left(-\vec{f} \times \frac{\partial \vec{f}}{\partial e}\right)$.}

Repeating the line of reasoning presented in the preceding calculation, we derive from (118), (63), and (164) the following:

$$
-\overrightarrow{\boldsymbol{f}} \times \frac{\partial \overrightarrow{\boldsymbol{f}}}{\partial e}=-\overrightarrow{\boldsymbol{f}} \times \overrightarrow{\mathbf{g}}\left(\frac{\partial t}{\partial E}\right)_{a, e, M_{o}}\left(\frac{\partial E}{\partial e}\right)_{t, e, M_{o}}=-\overrightarrow{\mathbf{w}} a^{2} \frac{\left(1-e^{2}\right)^{3 / 2}}{1+e \cos \nu}\left(\frac{\partial E}{\partial e}\right)_{t, a, M_{o}} .
$$

To compute $\partial E / \partial e$, one can start with the Kepler equation,

$$
E-e \sin E=M_{o}+n\left(t-t_{o}\right)
$$

which yields, for $t, a, M_{o}$ being fixed:

$$
d E-e \cos E d E-\sin E d e=0,
$$


insertion of (105) wherein will entail

$$
\left(\frac{\partial E}{\partial e}\right)_{t, a, M_{o}}=\frac{\sin E}{1-e \cos E}=\frac{\sin \nu}{\sqrt{1-e^{2}}} .
$$

All in all,

$$
-\overrightarrow{\boldsymbol{f}} \times \frac{\partial \overrightarrow{\boldsymbol{f}}}{\partial e}=-\overrightarrow{\mathbf{w}} a^{2} \frac{\left(1-e^{2}\right)}{1+e \cos \nu} \sin \nu
$$

\section{A.11 Calculation of $\dot{\overrightarrow{\boldsymbol{\mu}}} \cdot\left(-\overrightarrow{\boldsymbol{f}} \times \frac{\partial \overrightarrow{\boldsymbol{f}}}{\partial \omega}\right)$.}

Evidently, this vector is perpendicular to the instantaneous orbital plane and is $\omega$ independent. A direct computation, based upon (99-101), will lead us to:

$$
\begin{aligned}
& \left(\frac{\partial \overrightarrow{\boldsymbol{f}}}{\partial \omega} \times \overrightarrow{\boldsymbol{f}}\right)_{1}=\frac{\partial f_{2}}{\partial \omega} f_{3}-\frac{\partial f_{3}}{\partial \omega} f_{2}= \\
& r^{2}[-\sin \Omega \sin (\omega+\nu)+\cos \Omega \cos (\omega+\nu) \cos i] \sin (\omega+\nu) \sin i- \\
& r^{2} \cos (\omega+\nu) \sin i[\sin \Omega \cos (\omega+\nu)+\cos \Omega \sin (\omega+\nu) \cos i]= \\
& a^{2} \frac{\left(1-e^{2}\right)^{2}}{(1+e \cos \nu)^{2}}[-\sin \Omega \sin i], \\
& \left(\frac{\partial \overrightarrow{\boldsymbol{f}}}{\partial \omega} \times \overrightarrow{\boldsymbol{f}}\right)_{2}=\frac{\partial f_{3}}{\partial \omega} f_{1}-\frac{\partial f_{1}}{\partial \omega} f_{3}= \\
& r^{2} \cos (\omega+\nu) \sin i[\cos \Omega \cos (\omega+\nu)-\sin \Omega \sin (\omega+\nu) \cos i]- \\
& r^{2}[-\cos \Omega \sin (\omega+\nu)-\sin \Omega \cos (\omega+\nu) \cos i] \sin (\omega+\nu) \sin i= \\
& a^{2} \frac{\left(1-e^{2}\right)^{2}}{(1+e \cos \nu)^{2}} \quad[\cos \Omega \sin i], \\
& \left(\frac{\partial \overrightarrow{\boldsymbol{f}}}{\partial \omega} \times \overrightarrow{\boldsymbol{f}}\right)_{3}=\frac{\partial f_{1}}{\partial \omega} f_{2}-\frac{\partial f_{2}}{\partial \omega} f_{1}=
\end{aligned}
$$


$r^{2}[-\cos \Omega \sin (\omega+\nu)-\sin \Omega \cos (\omega+\nu) \cos i][\sin \Omega \cos (\omega+\nu)+\cos \Omega \sin (\omega+\nu) \cos i]-$ $r^{2}[-\sin \Omega \sin (\omega+\nu)+\cos \Omega \cos (\omega+\nu) \cos i][\cos \Omega \cos (\omega+\nu)-\sin \Omega \sin (\omega+\nu) \cos i]$

$$
=-r^{2} \cos i=-a^{2} \frac{\left(1-e^{2}\right)^{2}}{(1+e \cos \nu)^{2}} \cos i
$$

Briefly,

$$
-\left(\vec{f} \times \frac{\partial \vec{f}}{\partial \omega}\right)=-\overrightarrow{\mathbf{w}} a^{2} \frac{\left(1-e^{2}\right)^{2}}{(1+e \cos \nu)^{2}}
$$

A.12. Calculation of $\dot{\vec{\mu}} \cdot\left(-\vec{f} \times \frac{\partial \vec{f}}{\partial \Omega}\right)$

$$
\left(\frac{\partial \overrightarrow{\boldsymbol{f}}}{\partial \Omega} \times \overrightarrow{\boldsymbol{f}}\right)_{1}=\frac{\partial f_{2}}{\partial \Omega} f_{3}-\frac{\partial f_{3}}{\partial \Omega} f_{2}=
$$

$r^{2}[\cos \Omega \cos (\omega+\nu)-\sin \Omega \sin (\omega+\nu) \cos i] \sin (\omega+\nu) \sin i=$ $a^{2} \frac{\left(1-e^{2}\right)^{2}}{(1+e \cos \nu)^{2}}[\cos \Omega \cos (\omega+\nu)-\sin \Omega \sin (\omega+\nu) \cos i] \sin (\omega+\nu) \sin i \quad$,

$$
\left(\frac{\partial \overrightarrow{\boldsymbol{f}}}{\partial \Omega} \times \overrightarrow{\boldsymbol{f}}\right)_{2}=\frac{\partial f_{3}}{\partial \Omega} f_{1}-\frac{\partial f_{1}}{\partial \Omega} f_{3}=
$$

$-r^{2}[-\sin \Omega \cos (\omega+\nu)-\cos \Omega \sin (\omega+\nu) \cos i] \sin (\omega+\nu) \sin i=$ $a^{2} \frac{\left(1-e^{2}\right)^{2}}{(1+e \cos \nu)^{2}}[\sin \Omega \cos (\omega+\nu)+\cos \Omega \sin (\omega+\nu) \cos i] \sin (\omega+\nu) \sin i$

$$
\left(\frac{\partial \overrightarrow{\boldsymbol{f}}}{\partial \Omega} \times \overrightarrow{\boldsymbol{f}}\right)_{3}=\frac{\partial f_{1}}{\partial \Omega} f_{2}-\frac{\partial f_{2}}{\partial \Omega} f_{1}=
$$




$$
\begin{gathered}
r^{2}[-\sin \Omega \cos (\omega+\nu)-\cos \Omega \sin (\omega+\nu) \cos i][\sin \Omega \cos (\omega+\nu)+\cos \Omega \sin (\omega+\nu) \cos i]- \\
r^{2}[\cos \Omega \cos (\omega+\nu)-\sin \Omega \sin (\omega+\nu) \cos i][\cos \Omega \cos (\omega+\nu)-\sin \Omega \sin (\omega+\nu) \cos i]= \\
r^{2}\left[-\cos ^{2}(\omega+\nu)-\sin ^{2}(\omega+\nu) \cos ^{2} i\right]=-a^{2} \frac{\left(1-e^{2}\right)^{2}}{(1+e \cos \nu)^{2}}\left[\cos ^{2}(\omega+\nu)+\sin ^{2}(\omega+\nu) \cos ^{2} i\right] .
\end{gathered}
$$

A.13. Calculation of $\overrightarrow{\boldsymbol{\mu}} \cdot\left(-\vec{f} \times \frac{\partial \vec{f}}{\partial i}\right)$

$$
\left(\frac{\partial \overrightarrow{\boldsymbol{f}}}{\partial i} \times \overrightarrow{\boldsymbol{f}}\right)_{1}=\frac{\partial f_{2}}{\partial i} f_{3}-\frac{\partial f_{3}}{\partial i} f_{2}=
$$

$r^{2}\{[-\cos \Omega \sin (\omega+\nu) \sin i] \sin (\omega+\nu) \sin i-$

$\sin (\omega+\nu) \cos i[\sin \Omega \cos (\omega+\nu)+\cos \Omega \sin (\omega+\nu) \cos i]\}=$

$$
a^{2} \frac{\left(1-e^{2}\right)^{2}}{(1+e \cos \nu)^{2}}[-\cos \Omega \sin (\omega+\nu)-\sin \Omega \cos (\omega+\nu) \cos i] \sin (\omega+\nu)
$$

$$
\left(\frac{\partial \overrightarrow{\boldsymbol{f}}}{\partial i} \times \overrightarrow{\boldsymbol{f}}\right)_{2}=\frac{\partial f_{3}}{\partial i} f_{1}-\frac{\partial f_{1}}{\partial i} f_{3}=
$$

$$
r^{2}\{\sin (\omega+\nu) \cos i[\cos \Omega \cos (\omega+\nu)-\sin \Omega \sin (\omega+\nu) \cos i]-
$$

$$
\sin \Omega \sin (\omega+\nu) \sin i \sin (\omega+\nu) \sin i\}=
$$

$$
a^{2} \frac{\left(1-e^{2}\right)^{2}}{(1+e \cos \nu)^{2}}[-\sin \Omega \sin (\omega+\nu)+\cos \Omega \cos (\omega+\nu) \cos i] \sin (\omega+\nu)
$$




$$
\left(\frac{\partial \overrightarrow{\boldsymbol{f}}}{\partial i} \times \overrightarrow{\boldsymbol{f}}\right)_{3}=\frac{\partial f_{1}}{\partial i} f_{2}-\frac{\partial f_{2}}{\partial i} f_{1}=
$$

$r^{2}[\sin \Omega \sin (\omega+\nu) \sin i][\sin \Omega \cos (\omega+\nu)+\cos \Omega \sin (\omega+\nu) \cos i]-$

$r^{2}[-\cos \Omega \sin (\omega+\nu) \sin i][\cos \Omega \cos (\omega+\nu)-\sin \Omega \sin (\omega+\nu) \cos i]=$

$r^{2} \sin (\omega+\nu) \cos (\omega+\nu) \sin i=a^{2} \frac{\left(1-e^{2}\right)^{2}}{(1+e \cos \nu)^{2}} \sin (\omega+\nu) \cos (\omega+\nu) \sin i$.

A.14. Calculation of $\overrightarrow{\boldsymbol{\mu}} \cdot\left(-\overrightarrow{\boldsymbol{f}} \times \frac{\partial \overrightarrow{\boldsymbol{f}}}{\partial M_{o}}\right)$

The Kepler equation (168) entails that

$$
\frac{\partial \overrightarrow{\boldsymbol{f}}}{\partial M_{o}}=\frac{1}{n} \quad \frac{\partial \overrightarrow{\boldsymbol{f}}}{\partial t}=\frac{a^{3 / 2}}{\sqrt{G m}} \overrightarrow{\mathrm{g}}
$$

and, therefore, with aid of (63) we obtain:

$$
-\overrightarrow{\boldsymbol{f}} \times \frac{\partial \overrightarrow{\boldsymbol{f}}}{\partial M_{o}}=-\overrightarrow{\mathrm{w}} \sqrt{G m a\left(1-e^{2}\right)} \frac{a^{3 / 2}}{\sqrt{G m}}=-\overrightarrow{\mathbf{w}} a^{2} \sqrt{1-e^{2}} .
$$

A.15. The terms $(\overrightarrow{\boldsymbol{\mu}} \times \overrightarrow{\boldsymbol{f}}) \cdot \frac{\partial}{\partial C_{j}}(\overrightarrow{\boldsymbol{\mu}} \times \overrightarrow{\boldsymbol{f}})$.

In the course of numerical computation, it is convenient to keep such terms as parts of the Hamiltonian (52). In case these terms are to be dealt with analytically, one will need the following general formulae valid for any $C_{j}, j=a, e, \Omega, \omega, i, M_{o}$ :

$$
\begin{aligned}
& (\overrightarrow{\boldsymbol{\mu}} \times \overrightarrow{\boldsymbol{f}}) \cdot \frac{\partial}{\partial C_{j}}(\overrightarrow{\boldsymbol{\mu}} \times \overrightarrow{\boldsymbol{f}})=(\overrightarrow{\boldsymbol{\mu}} \times \overrightarrow{\boldsymbol{f}}) \cdot\left(\frac{\partial \overrightarrow{\boldsymbol{\mu}}}{\partial C_{j}} \times \overrightarrow{\boldsymbol{f}}\right)+(\overrightarrow{\boldsymbol{\mu}} \times \overrightarrow{\boldsymbol{f}}) \cdot\left(\overrightarrow{\boldsymbol{\mu}} \times \frac{\partial \overrightarrow{\boldsymbol{f}}}{\partial C_{j}}\right) \\
& \approx(\overrightarrow{\boldsymbol{\mu}} \times \overrightarrow{\boldsymbol{f}}) \cdot\left(\overrightarrow{\boldsymbol{\mu}} \times \frac{\partial \overrightarrow{\boldsymbol{f}}}{\partial C_{j}}\right),
\end{aligned}
$$


the neglect of the term with $\partial \overrightarrow{\boldsymbol{\mu}} / \partial C_{j}$ being legitimate, because this input is of a even higher order than the other one 17 A direct algebraic calculation then yields, under the said approximation:

$$
\begin{aligned}
& (\overrightarrow{\boldsymbol{\mu}} \times \overrightarrow{\boldsymbol{f}}) \cdot \frac{\partial}{\partial C_{j}}(\overrightarrow{\boldsymbol{\mu}} \times \overrightarrow{\boldsymbol{f}})=\left(\mu_{2}^{2}+\mu_{3}^{2}\right) f_{1} \frac{\partial f_{1}}{\partial C_{j}}+\left(\mu_{3}^{2}+\mu_{1}^{2}\right) f_{2} \frac{\partial f_{2}}{\partial C_{j}}+\left(\mu_{1}^{2}+\mu_{2}^{2}\right) f_{3} \frac{\partial f_{3}}{\partial C_{j}} \\
& -\mu_{1} \mu_{2}\left(f_{1} \frac{\partial f_{2}}{\partial C_{j}}+\frac{\partial f_{1}}{\partial C_{j}} f_{2}\right)-\mu_{2} \mu_{3}\left(f_{2} \frac{\partial f_{3}}{\partial C_{j}}+\frac{\partial f_{2}}{\partial C_{j}} f_{3}\right)-\mu_{3} \mu_{1}\left(f_{3} \frac{\partial f_{1}}{\partial C_{j}}+\frac{\partial f_{1}}{\partial C_{j}} f_{3}\right),
\end{aligned}
$$

expression for $f_{j}$ given above by (99-101)

17 The physical meaning of $\partial \overrightarrow{\boldsymbol{\mu}} / \partial C_{j}$ is the influence of the satellite upon the precession rate of the primary. As demonstrated by Laskar (2004), this effect is of a higher order of smallness. 


\section{References}

[1] Arnold, V. I. 1989. Mathematical Methods of Classical Mechanics. Springer-Verlag, NY 1989.

[2] Ashby, N., and T. Allison. 1993. "Canonical Planetary Perturbation Equations for Velocity-Dependent Forces, and the Lense-Thirring Precession." Celestial Mechanics and Dynamical Astronomy, Vol. 57, pp. 537 - 585.

[3] Brouwer, D. 1959. "Solution of the problem of artificial satellite theory without drag." The Astronomical Journal, Vol. 64, pp. 378 - 397.

[4] Brouwer, D., and G. M. Clemence. 1961. Methods of Celestial Mechanics. Academic Press, NY \& London. (Chapter XI)

[5] Brumberg, V. A., L. S. Evdokimova, \& N. G. Kochina. 1971. "Analytical Methods for the Orbits of Artificial Satellites of the Moon." Celestial Mechanics, Vol. 3, pp. 197 - 221.

[6] Brumberg, V.A. 1992. Essential Relativistic Celestial Mechanics. Adam Hilger, Bristol.

[7] Burns, J., \& V.Safronov 1973, "Asteroid Nutation Angles." The Monthly Notices of the Royal Astronomical Society of London, Vol. 165, pp. 403 - 411

[8] Chambers, J. E. 1999. "A Hybrid Symplectic integrator that Permits Close Encounters between Massive Bodies." The Monthly Notices of the Royal Astronomical Society of London, Vol. 304, pp. 793 - 799.

[9] Chernoivan, V. A., \& Mamaev, I. S. 1999. "The Restricted Two-Body Problem and the Kepler Problem in the Constant-Curvature Spaces." Regular and Chaotic Dynamics, Vol. 4, pp. $112-124$.

[10] Defraigne, P.; Rivoldini, A.; Van Hoolst, T.; Dehant, V. 2003. "Mars nutation resonance due to Free Inner Core Nutation." Journal of Geophysical Research, Vol. 108, p. 5128

[11] Dehant, V.; van Hoolst, T.; Defraigne, P. 2000. "Comparison between the nutations of the planet Mars and the nutations of the Earth." Surveys in Geophysics, Vol. 21, pp. 89 - 110 .

[12] Duncan, M. J.; Levison, H. F. \& M. H. Lee. 1995. "A Multiple Time Step Symplectic Algorithm for Integrating Close Encounters." The Astronomical Journal, Vol. 116, pp. $2067-2077$

[13] Efroimsky, M. 2001. "Relaxation of Wobbling Asteroids and Comets. Theoretical Problems. Perspectives of Experimental Observation." Planetary \& Space Science, Vol. 49, pp. $937-955$

[14] Efroimsky, M. 2002a. "Equations for the orbital elements. Hidden symmetry." Preprint No 1844 of the Institute of Mathematics and its Applications, University of Minnesota http://www.ima.umn.edu/preprints/feb02/feb02.html

[15] Efroimsky, M. 2002b. "The Implicit Gauge Symmetry Emerging in the N-body Problem of Celestial Mechanics." astro-ph/0212245 
[16] Efroimsky, M. 2002c. "Euler, Jacobi, and Missions to Comets and Asteroids." Advances in Space Research, Vol. 29, pp. 725 - 734

[17] Efroimsky, M., \& P. Goldreich. 2003. "Gauge Symmetry of the N-body Problem in the Hamilton-Jacobi Approach." Journal of Mathematical Physics, Vol. 44, pp. 5958 - 5977 astro-ph/0305344

[18] Efroimsky, M., \& Goldreich, P. 2004. "Gauge Freedom in the N-body Problem of Celestial Mechanics." Astronomy \& Astrophysics, Vol. 415, pp. 1187 - 1199 astro-ph/0307130

[19] Eubanks, T. M. 1993. "Variation in the orientation of the Earth. In: Contributions of Space Geodesy to Geodynamics: Earth Dynamics, pp. 1-54, Amer. Geophys. Union, Washington, 1993.

[20] Goldreich, P. 1965. "Inclination of satellite orbits about an oblate precessing planet." The Astronomical Journal, Vol. 70, p. 5 - 9

[21] Gradshtein, I. S., and I. M. Ryzhik. 1994. Tables of Integrals, Series, and Products. Academic Press, NY.

[22] Hill, G. W. 1913. "Motion of a System of Material Points under the Action of Gravitation." The Astronomical Journal, Vol. 27, p. 171 - 182

[23] Kinoshita, T. 1993. "Motion of the Orbital Plane of a Satellite due to a Secular Change of the Obliquity of its Mother Planet." Celestial Mechanics and Dynamical Astronomy, Vol. 57, pp. $359-368$

[24] Kozai, Y. 1960. "Effect of precession and nutation on the orbital elements of a close earth satellite." The Astronomical Journal, Vol. 65, pp. 621 - 623.

[25] Lagrange, J.-L. 1778. Sur le Problème de la détermination des orbites des comètes d'après trois observations, 1-er et 2-ième mémoires. Nouveaux Mémoires de l'Académie de Berlin, 1778. Later edition: In Euvres de Lagrange. Vol. IV, Gauthier-Villars, Paris 1869 .

[26] Lagrange, J.-L. 1783. Sur le Problème de la détermination des orbites des comètes d'après trois observations, 3-ième mémoire. Ibidem, 1783. Later edition: In Euvres de Lagrange. Vol. IV, Gauthier-Villars, Paris 1869.

[27] Lagrange, J.-L. 1808. "Sur la théorie des variations des éléments des planètes et en particulier des variations des grands axes de leurs orbites". Lu, le 22 août 1808 à l'Institut de France. Later edition: In Euvres de Lagrange. Vol. VI, p. 713 - 768, Gauthier-Villars, Paris 1877.

[28] Lagrange, J.-L. 1809. "Sur la théorie générale de la variation des constantes arbitraires dans tous les problèmes de la mécanique". Lu, le 13 mars 1809 à l'Institut de France. Later edition: In Euvres de Lagrange. Vol.VI, p.771-805, Gauthier-Villars, Paris 1877.

[29] Lagrange, J.-L. 1810. "Second mémoire sur la théorie générale de la variation des constantes arbitraires dans tous les problèmes de la mécanique". Lu, le 19 février 1810 à l'Institut de France. Later edition: In Euvres de Lagrange. Vol. VI, p. 809 - 816, GauthierVillars, Paris 1877. 
[30] Laskar, J. 1990. "The Chaotic Motion of the Solar System. A Numerical Estimate of the Size of the Chaotic Zones." Icarus, Vol. 88, pp. 266 - 291.

[31] Laskar, J. 2004. "A Comment on "Accurate Spin Axes and Solar System Dynamics: Climatic Variations for the Earth and Mars"." Astronomy \& Astrophysics, Vol. 416, pp. $799-800$.

[32] Laskar, J., \& J. Robutel. 1993. "The Chaotic Obliquity of the Planets." Nature, Vol. 361, pp. $608-612$.

[33] Marsden, J., \& T. Ratiu. 2003. Introduction to Mechanics and Symmetry. Springer, NY 2003

[34] Molina, A., F. Moreno, \& F. Martinez-López. 2003. "Energy dissipation by internal stresses in a free-rotating symmetric ellipsoid: Application to Comet P/Halley." Astronomy $\&$ Astrophysics, Vol. 398, pp. 809 - 817

[35] Morbidelli, A. 2002. Modern Celestial Mechanics: Dynamics in the Solar System. Taylor \& Francis, London 2002.

[36] Murison, M. 1988. "Satellite Capture and the Restricted Three-Body Problem." Ph.D. Thesis, University of Wisconsin, Madison 1988.

[37] Newman, W., \& M. Efroimsky. 2003. "The Method of Variation of Constants and Multiple Time Scales in Orbital Mechanics." Chaos, Vol. 13, pp. 476 - 485.

[38] Pang, K. D., J. B. Pollack, J. Veverka, A. L. Lane, \& J. M. Ajello. 1978. "The Composition of Phobos: Evidence for Carbonateous Chondrite Surface from Spectral Analysis." Science, Vol. 199, p. 64

[39] Pollack, J. B.; Burns, J. A.; \& Tauber, M. E. 1979. "Gas Drag in Primordial Circumplanetary Envelopes. A Mechanism for Satellite Capture." Icarus, Vol. 37, p. 587

[40] Prendergast, K. H. 1958. "The Effects of Imperfect Elasticity in Problems of Celestial Mechanics." Astronomical Journal, Vol. 63, pp. 412- 414

[41] Proskurin, V. F., \& Batrakov, Y. V. 1960. "Perturbations of the Motion of Artificial Satellites, caused by the Earth Oblateness." The Bulletin of the Institute of Theoretical Astronomy, Vol. 7, pp. 537 - 548.

[42] Richardson, D. L., and Kelly, T. J. 1988. "Two-body motion in the post-Newtonian approximation." Celestial Mechanics, Vol. 43, pp. 193-210.

[43] Sharaf, S. G. \& N. A. Budnikova. 1969a. "On Secular Perturbations in the Elements of the Earth's Orbit and their Influence on the Climates in the Geological Past." Bulletin of the Leningrad Institute of Theoretical Astronomy. Vol. 11, pp. 231 - 261. /in Russian/

[44] Sharaf, S. G. \& Budnikova. 1969b. "Secular Perturbations in the Elements of the Earth's Orbit and the Astronomical Theory of Climate Variations" Proceedings of the Leningrad Institute of Theoretical Astronomy. Vol. 14, pp. 48 - 84. /in Russian/ 
[45] Sharma, I., J. A. Burns, \& C.-Y. Hui. 2004. "Nutational Damping Times in Solids of Revolution." To be published in the Monthly Notices of the Royal Astronomical Society of London.

[46] Slabinski, V. 2003. "Satellite Orbit Plane Perturbations Using an Efroimsky Gauge Velocity." Talk at the 34th Meeting of the AAS Division on Dynamical Astronomy, Cornell University, May 2003.

[47] Szebehely, V. 1967. Theory of Orbits. Academic Press, NY.

[48] Tolson, R. H., and 15 collaborators. 1978. "Viking First Encounter of Phobos. Preliminary Results." Science, Vol. 199, p. 61

[49] Touma, J., \& J. Wisdom. 1994. "Lie-Poisson Integrators for Rigid Body Dynamics in the Solar System." The Astronomical Journal, Vol. 107, pp. 1189 - 1202.

[50] Van Hoolst, T.; Dehant, V.; Defraigne, P. 2000. "Chandler wobble and Free Core Nutation for Mars." Planetary and Space Science, Vol. 48, pp. 1145 - 1151.

[51] Vernekar, A. D. 1972. "Long-period Global Variations of the Incoming Solar Radiation." Meteor. Monogr., Vol. 12, pp. 1 - 21.

[52] Veverka, J. 1977. "Phobos and Deimos." Scientific American, Vol. 236, p. 30

[53] Ward, W. 1973. "Large-scale Variations in the Obliquity of Mars." Science. Vol. 181, pp. $260-262$.

[54] Ward, W. 1974. "Climatic Variations of Mars. Astronomical Theory of Insolation." Journal of Geophys. Research, Vol. 79, pp. 3375 - 3386.

[55] Ward, W. 1979. "Present Obliquity Oscillations of Mars - Fourth-order Accuracy in Orbital e and i." Journal of Geophys. Research, Vol. 84, pp. 237 - 241.

[56] Ward, W. 1982. "Comments on the long-term Stability of the Earth's Obliquity." Icarus, Vol. 50, pp. $444-448$.

[57] Waz, P. 1999. "Disturbing Function in the Analytical Theory of the Motion of Phobos." The Astronomical Journal, Vol. 348, pp. 300 - 310 